\title{
The Axiomatic Translation Principle for Modal Logic
}

\author{
RENATE A. SCHMIDT \\ University of Manchester \\ and \\ ULLRICH HUSTADT \\ University of Liverpool
}

\begin{abstract}
In this paper we present a translation principle, called the axiomatic translation, for reducing propositional modal logics with background theories, including triangular properties such as transitivity, Euclideanness and functionality, to decidable fragments of first-order logic. The goal of the axiomatic translation principle is to find simplified theories, which capture the inference problems in the original theory, but in a way that can be readily automated and is easier to deal with by existing (first-order) theorem provers than the standard translation. The principle of the axiomatic translation is conceptually very simple and can be almost completely automated. Soundness is automatic under reasonable assumptions, general decidability results can be stated and termination of ordered resolution is easily achieved. The non-trivial part of the approach is proving completeness. We prove results of completeness, decidability, model generation, the small model property and the interpolation property for a number of common and less common modal logics. We also present results of experiments with a number of first-order logic theorem provers which are very encouraging.
\end{abstract}

Categories and Subject Descriptors: F.4.1 [Mathematical Logic]: Modal Logic, Proof theory; I.2.3 [Deduction and Theorem Proving]: Deduction, Resolution; I.2.4 [Knowledge Representation Formalisms and Methods]: Modal logic, Predicate logic

General Terms: Algorithms, Performance, Theory

Additional Key Words and Phrases: Translation approach, decidability, completeness, small model property

\section{INTRODUCTION}

Modal logic provides an appropriate formal framework for an ever increasing number of different application areas of computer science. In the field of knowledge representation, particularly in the subfield concerned with description logics, modal logics arise in the form of the basic multi-modal logic and propositional dynamic logic. Modal logics form the basis of many agent logics. Various aspects of agents are formalised by modal operators, e.g. $S 5$ modalities for knowledge, $K D 45$ modal-

Auhtors' addresses: R. A. Schmidt, School of Computer Science, University of Manchester, United Kingdom, Renate.Schmidt@cs.manchester.ac.uk, U. Hustadt, Department of Computer Science, University of Liverpool, United Kingdom, U.Hustadt@csc.liv.ac.uk.

Permission to make digital/hard copy of all or part of this material without fee for personal or classroom use provided that the copies are not made or distributed for profit or commercial advantage, the ACM copyright/server notice, the title of the publication, and its date appear, and notice is given that copying is by permission of the ACM, Inc. To copy otherwise, to republish, to post on servers, or to redistribute to lists requires prior specific permission and/or a fee.

(C) 2005 ACM 1529-3785/2005/0700-0001 $\$ 5.00$ 
ities for belief, and so on. Normally agent logics also include operators formalising notions such as ability and commitment which are given by non-standard modal operators. Agent-based systems are a particular application area, where there is a clear need for non-standard modal logics, that is, modal logics that do not just simply coincide with familiar modal logics. There is therefore an increasing interest in non-standard combinations of modal logics. These modal logics are commonly given through a set of modal axiom schemas in the form of a Hilbert axiomatisation. Anyone who has studied Hilbert-style axiomatisations of modal logics or other logics knows that, except for very simple theorems, it is difficult to derive theorems in this framework. With lots of practice one can become quite good at doing Hilbert-style proofs, but in general, writing down proofs using this kind of approach requires a lot of skill. What makes this syntactic approach difficult to use is that it is a bottom-up approach. One needs to guess with which axioms to start and which instances of the inference rules to apply during the deduction. This is also why Hilbert-style proof search is difficult to automate effectively. While Hilbert-style deduction is easy to automate by reduction to first-order logic, due to the absence of the subformula property the search space is enormous. Consequently, there are currently no effective implementations of Hilbert systems. The question arises how effective reasoning systems can be constructed for a given combination of (possibly interacting) modal logics. The most common approach is to adopt a semantics-based method. The popular tableau approaches, and many sequent-style approaches, can be seen as methods for constructing a Kripke model for a given formula. Another option is to use a semantics-based translation into first-order or second-order logic and then to use first-order or second-order theorem provers [Ohlbach et al. 2001]. In all these cases it is necessary to find a class of Kripke models with respect to which the given Hilbert axiomatisation is sound and complete. Again one would want to automate this process, or at least have some sort of automated support. A number of automated second-order quantifier elimination procedures were developed and studied that transform Hilbert axioms into corresponding properties of the accessibility relation in the Kripke semantics of modal logics (cf. Nonnengart et al. [1997]).

The present paper investigates an alternative approach to automated reasoning for modal logic. The idea is based on an approach in which, starting from a Hilbert axiomatisation, by a process of partial translation and proof-theoretic transformation we can directly obtain proof methods for a wide variety of modal logics. We introduce and study a principle, called the axiomatic translation principle, of reducing modal logic problems into first-order logic. What we try to achieve with the axiomatic translation principle is to find a simplified theory, which captures the semantic background theory of a given modal logic in a new way, which has both theoretical and practical advantages. The axiomatic translation principle is conceptually very simple; it is based on the standard relational translation method, but instead of using correspondence properties, the axiomatic translation principle incorporates modal axioms into the first-order translation of modal formulae. This provides a method for avoiding having to handle properties such as transitivity, Euclideanness and functionality, which are difficult to deal with efficiently by current first-order logic theorem provers. In general, it is not known how to use first-order logic theo- 
rem provers as decision procedures for these properties. For example, no resolution decision procedures are known for decidable first-order logic fragments relevant to modal logics which include the formula $\forall x y z(R(x, y) \wedge R(x, z) \rightarrow R(y, z))$ expressing the Euclideanness of a relation $R$. The clauses in the clausal form of this formula, and also Euclideanness and functionality, contain no single maximal literal under common orderings. This means these clauses are eligible for many more inferences compared to clauses which contain just one maximal literal. Examples of the latter kind are properties such as reflexivity, symmetry and seriality. One contribution of this paper is overcoming the difficulties of using correspondence properties. Modal axiom schemas are incorporated into the translation of a modal logic problem by partial instantiations of the modal axiom schema, which are given by partial instantiations of so-called schema clauses. These schema clauses can be automatically deduced from the modal axioms, and we show that soundness of the presented method is automatic under some weak, reasonable assumptions. More challenging is proving completeness. First, a bounded set of instantiations of the schema clauses needs to be identified. Second, in our approach to proving completeness, we need to prove that it is possible to simulate hyperresolution refutations based on the relational translation using classical correspondence properties with the axiomatic approach. The reward for succeeding in proving completeness is automatic decidability, and other nice properties such as interpolation and sound and complete tableau systems. Besides proving completeness everything else can be easily automated and implemented. While in this paper we focus on the application of the axiomatic translation to well-known axiom schemas, it is clear that the principle and the insights underlying the axiomatic translation are applicable to a wide range of non-standard axiom schemas. This could turn out to be especially useful in applications, for instance, agent-based systems and description logics, as well as related fragments of first-order logic. For example the principle can be used to obtain decision procedures for the monadic two-variable guarded fragment with transitivity introduced in Ganzinger, Meyer and Veanes [1999].

The paper is structured as follows. Section 2 gives an informal introduction and overview of the principle of the axiomatic translation. The axiomatic translation is then defined in two steps. First a standard semantics-based translation of modal formulae is defined in Section 3. Then in Section 4 the axiomatic translation is specified by incorporating modal axioms into the standard translation. General soundness results are proved for a pure form of the axiomatic translation and a mixed form where the classical translation using correspondence properties and the axiomatic translation are used together. In Section 5 completeness results are presented for a series of familiar modal logics and modal logics extended with generalised modal axioms. Since the axiomatic translation reduces modal logics to decidable first-order fragments and decidable clausal classes, general conditions can be identified under which modal logics are decidable. Results of decidability, decidability by resolution, model generation and the small model property are presented in Section 6. The results of practical experience with first-order theorem provers is reported in Section 7. Section 8 discusses further consequences and related work, and Section 9 is the conclusion.

It is assumed that the reader has a basic familiarity with modal logic, first-order 
logic and resolution. The notation used in this paper is the same as in the survey papers [De Nivelle et al. 2000; Schmidt and Hustadt 2003]. For easy reference Appendices A and B give a summary of the basics of modal logic and clause logic, and define the hyperresolution calculus used in the completeness proofs. The formal proofs of results in this paper are given in Appendices $\mathrm{C}-\mathrm{G}$.

\section{THE IDEA OF THE AXIOMATIC TRANSLATION PRINCIPLE}

The idea of the principle presented in this paper can be situated in the general framework of Ohlbach [1998] for the combination of Hilbert-style deduction and semantic reasoning for non-classical logics. In this framework, if a first-order semantics is known for some of the logical connectives in such logics, the semantic definitions are used as rewrite rules for eliminating these connectives. In addition, Hilbert axioms and rules are encoded directly with quantification over formula variables. The formulas are left as terms and are treated in a special way. Thus a problem specification has four components, the semantic rewrite rules determining the semantic encoding of some of the logical operators, the specification of a semantic structure, for example, in the form of frame correspondence properties, the encoded Hilbert axioms and rules, and finally the conjecture to be proved or refuted. The result is a first-order formulation of the problem and now ordinary first-order theorem proving methods can be applied.

To explain the translation principle, we give more details of the Ohlbach framework. Let 'holds' be a designated predicate capturing the semantic definition of the logical operators $\neg, \wedge, \vee, \rightarrow, \leftrightarrow$ and $\square$ by the following first-order equivalences:

$$
\begin{aligned}
\forall p \forall x(\operatorname{holds}(\neg p, x) & \leftrightarrow \neg \operatorname{holds}(p, x)) \\
\forall p q \forall x(\operatorname{holds}(p \star q, x) & \leftrightarrow(\operatorname{holds}(p, x) \star \operatorname{holds}(q, x))) \\
\forall p \forall x(\operatorname{holds}(\square p, x) & \leftrightarrow \forall y(R(x, y) \rightarrow \operatorname{holds}(p, y)))
\end{aligned}
$$

where $\star \in\{\wedge, \vee, \rightarrow, \leftrightarrow\}$. Let $S$ be the (finite) set of these equivalences, and let $\Delta$ be a (finite) set of modal axioms. A T-encoded problem specification of the satisfiability of $\varphi$ in a (multi-)modal logic $K_{(m)} \Delta$ in the Ohlbach framework is given by

$$
\operatorname{tr}_{K_{(m)} \Delta}(\varphi)=\exists \bar{p} \exists x \operatorname{holds}(\varphi, x) \wedge \bigwedge S \wedge \bigwedge H .
$$

$H$ is the (finite) set of $T$-encodings of the axioms in $\Delta$. For any axiom $\mathcal{A}$ in $\Delta$, its $T$-encoding is defined by $\forall \bar{p} \forall x \operatorname{holds}(\mathcal{A}, x)$, where $\bar{p}$ are the propositional variables occurring in $\mathcal{A}$. (Ohlbach also allows mixed semantics and $T$-encoded problem specifications.)

Theorem 2.1 Let $\varphi$ be a modal formula, let $\Delta$ be a finite set of modal logic formulae and let $H$ be the set of $T$-encodings of $\Delta$. Suppose $K_{(m)} \Delta$ is sound and complete. Then, $\varphi$ is satisfiable in $K_{(m)} \Delta$ iff $\operatorname{tr}_{K_{(m)}} \Delta(\varphi)$ is first-order satisfiable.

Note that the modus ponens inference rule is not part of $\operatorname{tr}_{K_{(m)} \Delta}(\varphi)$. Thus, when showing the satisfiability/validity of $\operatorname{tr}_{K_{(m)} \Delta}(\varphi)$ we are not simply constructing Hilbert-style proofs in first-order logic.

The equivalences in $S$ can be used as rewrite rules to transform the candidate formula to be proved and also the axioms in $H$. For example, if the candidate 
formula is $\varphi=\square \neg \square p$ then $\exists p \exists x \operatorname{holds}(\varphi, x)$ transforms to

$$
\exists p \exists x \forall y(R(x, y) \rightarrow \neg(\forall z R(y, z) \rightarrow \operatorname{holds}(p, z))),
$$

by exhaustively applying the equivalences (1)-(3) from left-to-right. Ohlbach calls this the $S$-normalised representation of $\varphi$. Hilbert axioms can be normalised in a similar fashion. For example, rewriting the $T$-encodings of the axioms $4=\square p \rightarrow$ $\square \square p$ and $T=\square p \rightarrow p$ one obtains:

$$
\begin{aligned}
& \forall p \forall x \operatorname{holds}(\square p \rightarrow \square \square p, x) \equiv \forall p \forall x(\operatorname{holds}(\square p, x) \rightarrow \operatorname{holds}(\square \square p, x)) \\
& \equiv \forall p \forall x(\operatorname{holds}(\square p, x) \rightarrow \forall y(R(x, y) \rightarrow \operatorname{holds}(\square p, y))) \\
& \equiv \forall p \forall x(\forall y(R(x, y) \rightarrow \operatorname{holds}(p, y)) \\
& \rightarrow \forall y(R(x, y) \rightarrow(\forall z(R(y, z) \rightarrow \operatorname{holds}(p, z))))) \\
& \forall p \forall x \operatorname{holds}(\square p \rightarrow p, x) \\
& \equiv \forall p \forall x(\operatorname{holds}(\square p, x) \rightarrow \operatorname{holds}(p, x)) \\
& \equiv \forall p \forall x(\forall y(R(x, y) \rightarrow \operatorname{holds}(p, y)) \rightarrow \operatorname{holds}(p, x))
\end{aligned}
$$

If we replace the holds $(p, x)$ literals in (5) and (7) by literals of the form $P(x)$ and the $\forall p$ quantifier by $\forall P$ then what we have is the standard translation of 4 and $T$. The standard translation of the axioms are thus second-order formulae, while the $T$-encodings of modal axioms are always first-order logic formulae (in which modal formulae are encoded as terms).

Since $K 4, K T$ and $S 4$ are decidable logics, an immediate question is: Which firstorder methods decide $T$-encoded problem specifications in these logics? There are a number of solvable first-order fragments which can be decided with first-order methods. These include the two-variable fragment, the guarded fragment [De Nivelle and de Rijke 1999; Ganzinger and de Nivelle 1999], Maslov's class $\bar{K}$ [Hustadt and Schmidt 1999b], and fluted logic [Schmidt and Hustadt 2000]. Evidently, (5) and (7) are formulae in more than two variables, and thus do not belong to $F O^{2}$. The usual techniques are not sufficient to reduce them to $F O^{2}$ either. Neither (5) nor (7) is a guarded formula, because of the absence of a guard literal in the quantification. Fluted logic is also not suitable for expressing (5) and (7), since no linear order can be defined over the variables and quantification to meet the syntactic requirements of the definition of fluted logic. While the formula for axiom $T$ belongs to Maslov's class $\bar{K}$, the formula for axiom 4 does not. Analysis of the respective clausal forms

$$
\begin{array}{ccc}
\text { for } 4: \quad & R\left(x, f_{4}(p, x)\right) \vee \neg R(x, y) \vee \neg R(y, z) \vee \operatorname{holds}(p, z) \\
& \neg \operatorname{holds}\left(p, f_{4}(p, x)\right) \vee \neg R(x, y) \vee \neg R(y, z) \vee \operatorname{holds}(p, z) \\
\text { for } T: \quad & R\left(x, f_{T}(p, x)\right) \vee \operatorname{holds}(p, x) \\
& \neg \operatorname{holds}\left(p, f_{T}(p, x)\right) \vee \operatorname{holds}(p, x)
\end{array}
$$

reveals also that the normalised $T$-encoding of the axiom 4 does not belong to any known solvable clausal classes. However, the normalised $T$-encoding of $T$ can be expressed, for example, in $D L^{*}$ [De Nivelle et al. 2000] or the clausal class of Maslov's class $\bar{K}$ [Hustadt and Schmidt 1999b].

The idea of the approach described in this paper is the following. Take a modal axiom, form its $T$-encoding, but instead of normalising all modal connectives away, 
do a partial normalisation which stops before the innermost modalities are eliminated, i.e. in the case of 4 and $T$ the normalisation process stops once (4) and (6) are generated. The clausal forms are:

$$
\begin{array}{lll}
\neg \operatorname{holds}(\square p, x) \vee \neg R(x, y) \vee \operatorname{holds}(\square p, y) & & \text { for } 4 \\
\neg \operatorname{holds}(\square p, x) \vee \operatorname{holds}(p, x) & & \text { for } T
\end{array}
$$

Now, if we regard $\square p$ and $p$ as ground terms then it is not difficult to see that both clauses belong to a large selection of solvable clausal classes and decidable first-order fragments, for example, $D L^{*}$ and the class of guarded clauses [Ganzinger and de Nivelle 1999]. They even belong to $F O^{2}$ and all other decidable fragments mentioned above. This can be more easily seen if the terms $\square p$ and $p$ are embedded in the predicate symbol, as in:

$$
\begin{aligned}
& \neg Q_{\square p}(x) \vee \neg R(x, y) \vee Q_{\square p}(y) \quad \text { and } \\
& \neg Q_{\square p}(x) \vee Q_{p}(x) .
\end{aligned}
$$

The way to view literals of the form $Q_{\psi}(x)$, is to think of them as saying that the formula $\psi$ holds at the world $x$. These clauses are examples of what we call schema clauses. The properties of a transitive (resp. reflexive) $\square$ operator are captured by including all (partial) instantiations of the propositional variables of the appropriate schema clause with modal formula terms. Clearly the set of all partial instantiations is infinite. However since proofs are finite, any proof will require only finitely many of the partial instantiations of the Hilbert-axioms and therefore of the schema clauses. If we knew which schema clause instances are essential for arbitrary proofs, then this would be extremely beneficial, since then we would get a decision procedure. Thus the main intuition of the axiomatic translation principle is to restrict the number of partial instantiations of the schema clauses to a finitely bounded set and incorporate the set into the translation of a candidate formula. For the translation to work the schema clauses must be linked with the translation of the candidate formula. This is achieved by basing the translation on a particular form of structural transformation.

It is instructive to consider an example. Suppose we want to test the satisfiability of the formula $\varphi=\square \neg \square p$ in $K$. (It is obviously satisfiable in $K$, but the example is deliberately kept small so that the idea of the principle is not obscured.) The following is a suitable structural form of the classical relational translation of $\varphi$. New symbols have been introduced for all $\square$ subformulae of $\varphi$. (It is not exactly the translation defined formally below, but serves to illustrate the principle.)

$$
\begin{array}{rlrl}
Q_{\square \neg \square p}(a) & & \\
\forall x\left(Q_{\square \neg \square p}(x)\right. & \left.\leftrightarrow \forall y\left(R(x, y) \rightarrow \neg Q_{\square p}(y)\right)\right) & & \text { definition for } \square \neg \square p \\
\forall x\left(Q_{\square p}(x)\right. & \left.\leftrightarrow \forall y\left(R(x, y) \rightarrow Q_{p}(y)\right)\right) & & \text { definition for } \square p
\end{array}
$$

The symbol $a$ denotes the Skolem constant representing the root world in a Kripke model. The translation used is satisfiability equivalence preserving. Thus, the formula $\varphi$ is satisfiable in $K$ iff the conjunction of the above three formulae is firstorder satisfiable. The satisfiability of $\square \neg \square p$ in $K$ can now be tested by applying a standard theorem prover to the above set.

ACM Transactions on Computational Logic, Vol. X, No. Y, August 2005. 
In order to test satisfiability in $K 4$, the standard approach is to add frame correspondence properties for $R$ as a theory to the translation of the candidate formula. However, we adapt the translation of the candidate formula by instead incorporating the axiom 4 into the translation. This is achieved by incorporating a finitely bounded number of instances of the schema clause of 4 .

$$
\neg Q_{\square p}(x) \vee \neg R(x, y) \vee Q_{\square p}(y) \quad \text { schema clause of } 4 \text { for } \square p
$$

It turns out that in the case of $K 4$ it is enough to restrict the instantiation set to one instance of the schema clause for every $\square$ subformula in the candidate formula. In the case of our running example we can limit the set to the following two instances, one for each $\square$ subformula of $\varphi=\square \neg \square p$.

$$
\begin{array}{ll}
\neg Q_{\square \neg \square p}(x) \vee \neg R(x, y) \vee Q_{\square \neg \square p}(y) & \text { schema } 4 \text { instance with } \square p / \square \neg \square p \\
\neg Q_{\square p}(x) \vee \neg R(x, y) \vee Q_{\square p}(y) & \text { schema } 4 \text { instance with } \square p / \square p
\end{array}
$$

(Here $\phi / \psi$ denotes the simultaneous replacement of the formula $\phi$ in a schema clause by the formula $\psi$.)

We refer to this form of encoding as the axiomatic translation (or axiomatic reduction) of $\varphi$ with respect to $K 4$. The general principle of the axiomatic approach for $K 4$ is the following. For every $\square$ subformula, $\square \psi$, of the candidate formula include the clause $\neg Q_{\square \psi}(x) \vee \neg R(x, y) \vee Q_{\square \psi}(y)$ in the translation, provided $\square$ is a 4-modality. Similarly for other axioms. For example, if $\square$ is a $T$-modality then for every $\square$ subformula $\square \psi$ of the candidate formula we need to include the clause $\neg Q_{\square \psi}(x) \vee Q_{\psi}(x)$.

The main difficulty of the axiomatic translation principle is to know how many and which instances of a schema clause are needed for completeness. In the Hilbert axiomatisation the axioms are valid for all substitution instances. Since we want to avoid the use of a substitution rule, we need to make sure that from the outset enough instances of the schema clauses are present in the translation of the candidate formula. Of course this does not preclude a lazy implementation which delays the translation of subformulae and the inclusion of substitution instances of Hilbert axioms until absolutely necessary.

\section{CLASSICAL TRANSLATION TO CLAUSE LOGIC}

In this paper, our definition of the axiomatic translation for modal logics is based on the standard relational semantics and structural transformation which introduced new symbols for modal formulae. We therefore first give a formal definition of the standard relational translation for modal logics and then define the axiomatic translation as an adaptation in the next section.

Without loss of generality, attention is restricted to modal formulae formulated in terms of the connectives $\wedge, \neg, \square$ and the constant $\perp$. It is assumed that all occurrences of double negation have been eliminated. If $\psi$ denotes a modal formula then, by definition, $\sim \psi$ denotes $\phi$ if $\psi=\neg \phi$, and $\neg \psi$ otherwise.

The standard translation into first-order logic is based on the standard Kripke semantics. Here, it is combined with structural transformation, that is, new symbols are introduced for subformulae of the candidate formula. Structural transformation (cf. for example [Boy de la Tour 1992; Plaisted and Greenbaum 1986]) is a wellknown technique and has a number of advantages. It enables the preservation of 


\begin{tabular}{|c|c|c|c|}
\hline $\mathcal{A}$ & Axiom & \multicolumn{2}{|c|}{ Correspondence property } \\
\hline$T$ & $\square p \rightarrow p$ & reflexivity & $\forall x R(x, x)$ \\
\hline 4 & $\square p \rightarrow \square \square p$ & transitivity & $\forall x y z(R(x, y) \wedge R(y, z) \rightarrow R(x, z))$ \\
\hline$B$ & $\diamond \square p \rightarrow p$ & symmetry & $\forall x y(R(x, y) \rightarrow R(y, x))$ \\
\hline$D$ & $\square p \rightarrow \diamond p$ & seriality & $\forall x \exists y R(x, y)$ \\
\hline alt $_{1}$ & $\diamond p \rightarrow \square p$ & functionality & $\forall x y z(R(x, y) \wedge R(x, z) \rightarrow y \approx z)$ \\
\hline 5 & $\diamond \square p \rightarrow \square p$ & Euclideanness & $\forall x y z(R(x, y) \wedge R(x, z) \rightarrow R(y, z))$ \\
\hline $4^{\kappa}$ & $\square p \rightarrow \square^{\kappa} \square p$ & & $\forall x y\left(R^{\kappa+1}(x, y) \rightarrow R(x, y)\right)$ \\
\hline alt $_{1}^{\kappa_{1}, \kappa_{2}}$ & $\diamond^{\kappa_{1}} \diamond p \rightarrow \square^{\kappa_{2}} \square p$ & & $\forall x y z\left(R^{\kappa_{1}+1}(x, y) \wedge R^{\kappa_{2}+1}(x, z) \rightarrow y \approx z\right)$ \\
\hline $5^{\kappa}$ & $\diamond^{\kappa} \square p \rightarrow \square p$ & & $\forall x y z\left(R^{\kappa}(x, y) \wedge R(x, z) \rightarrow R(y, z)\right)$ \\
\hline
\end{tabular}

Fig. 1. Axiom schemas and relational background theories $\left(\kappa \geq 1\right.$ and $\left.\kappa_{1}, \kappa_{2} \geq 0\right)$

the structure of the original formula in the first-order clausal form, thus improving the readability of first-order resolution proofs (cf. [Hustadt and Schmidt 2002]). When using hyperresolution and splitting, as is done in the completeness proofs of this paper, there is an almost one-to-one correspondence between resolution proofs and modal tableau proofs. Structural transformation can also be used to keep the complexity of the reduction from one logic into another low.

The translation of a modal logic formula $\varphi$ into first-order logic based on the classical approach can be specified as follows. For any subformula $\psi$ of $\varphi$, let the notation $\operatorname{Def}(\psi)$ represent the definition of $Q_{\psi}$, which is defined by:

$$
\begin{aligned}
\operatorname{Def}(\psi)= & \forall x\left(Q_{\psi}(x) \rightarrow \pi(\psi, x)\right) \\
& \wedge \forall x\left(Q_{\psi}(x) \rightarrow \neg Q_{\sim \psi}(x)\right) \\
& \wedge \forall x\left(Q_{\sim \psi}(x) \rightarrow \pi(\sim \psi, x)\right) .
\end{aligned}
$$

$Q_{\psi}$ is a new predicate symbols uniquely associated with the modal formula $\psi$, and $\pi(\psi, x)$ is a first-order formula with one free variable $x$ given by the following.

$$
\begin{aligned}
& \pi(\perp, x)=\perp \quad \pi(p, x)=\top \quad \pi(\neg p, x)=\neg Q_{p}(x) \\
& \pi(\psi \wedge \phi, x)=Q_{\psi}(x) \wedge Q_{\phi}(x) \quad \pi(\neg(\psi \wedge \phi), x)=Q_{\sim \psi}(x) \vee Q_{\sim \phi}(x) \\
& \pi(\square \psi, x)=\forall y\left(R(x, y) \rightarrow Q_{\psi}(y)\right) \quad \pi(\neg \square \psi, x)=\exists y\left(R(x, y) \wedge Q_{\sim \psi}(y)\right)
\end{aligned}
$$

Thus, $\pi$ is a function associating a first-order formula with any modal formula. Here the definition of $\pi$ is based on the standard relational semantics. The symbol $y$ denotes an arbitrary variable distinct from $x$. (It is not a mistake that $\pi(p, x)=\top$.)

Now let $\Pi$ be the mapping defined by:

$$
\Pi(\varphi)=\exists x Q_{\varphi}(x) \wedge \operatorname{Simpl}(\bigwedge\{\operatorname{Def}(\psi) \mid \psi \in \operatorname{Sf}(\varphi)\}),
$$

where $\operatorname{Sf}(\varphi)$ denotes the set of all subformulae of $\varphi$. Though not strictly necessary, the purpose of Simpl is to eliminate obvious redundancies in the definitional forms which can be dealt with in linear time, for example, deletion of trivially tautologous formulae such as $\forall x\left(Q_{p}(x) \rightarrow \top\right)$.

Lemma 3.1 For any modal formula $\varphi, \Pi(\varphi)$ can be computed in linear time.

If $\Delta$ is a set of first-order definable modal axioms, then let $\operatorname{Corr}(\Delta)$ denote the relational background theory corresponding to $\Delta$, that is, $\psi \in \operatorname{Corr}(\Delta)$ iff $\psi$ is the 


\begin{tabular}{|rl|rl|}
\hline$\neg \psi$ & $\neg Q_{\neg \psi}(x) \vee \neg Q_{\psi}(x)$ & $\neg(\psi \wedge \phi)$ & $\neg Q_{\neg(\psi \wedge \phi)}(x) \vee Q_{\sim \psi}(x) \vee Q_{\sim \phi}(x)$ \\
$\perp$ & $\neg Q_{\perp}(x)$ & $\square \psi$ & $\neg Q_{\square \psi}(x) \vee \neg R(x, y) \vee Q_{\psi}(y)$ \\
$\psi \wedge \phi$ & $\neg Q_{\psi \wedge \phi}(x) \vee Q_{\psi}(x)$ & $\neg \square \psi$ & $\neg Q_{\neg \square \psi}(x) \vee R\left(x, f_{\neg \square \psi}(x)\right)$ \\
& $\neg Q_{\psi \wedge \phi}(x) \vee Q_{\phi}(x)$ & & $\neg Q_{\neg \square \psi}(x) \vee Q_{\sim \psi}\left(f_{\neg \square \psi}(x)\right)$ \\
\hline
\end{tabular}

Fig. 2. Definitional clausal forms

\begin{tabular}{|cll|}
\hline $\mathcal{A}$ & Axiom & Schema clause $\mathrm{Ax}^{\mathcal{A}}(p)$ \\
\hline$T$ & $\square p \rightarrow p$ & $\neg Q_{\square p}(x) \vee Q_{p}(x)$ \\
4 & $\square p \rightarrow \square \square p$ & $\neg Q_{\square p}(x) \vee \neg R(x, y) \vee Q_{\square p}(y)$ \\
$B$ & $\neg \square \neg \square p \rightarrow p$ & $\neg R(x, y) \vee \neg Q_{\square p}(y) \vee Q_{p}(x)$ \\
$D$ & $\square p \rightarrow \neg \square \neg p$ & $\neg Q_{\square p}(x) \vee Q_{\neg \square \neg p}(x)$ \\
alt $t_{1}$ & $\neg \square p \rightarrow \square \neg p$ & $\neg Q_{\neg \square p}(x) \vee Q_{\square \neg p}(x)$ \\
5 & $\neg \square \neg \square p \rightarrow \square p$ & $\neg R(x, y) \vee \neg Q_{\square p}(y) \vee Q_{\square p}(x)$ \\
$4^{\kappa}$ & $\square p \rightarrow \square{ }^{\kappa} \square p$ & $\neg Q_{\square p}(x) \vee \neg R^{\kappa}(x, y) \vee Q_{\square p}(y)$ \\
$a_{1}^{\kappa_{1}, \kappa_{2}}$ & $\neg \square^{\kappa_{1}} \square p \rightarrow \square \square^{\kappa_{2}} \square \neg p$ & $\neg R^{\kappa}(x, y) \vee \neg Q_{\neg \square \psi}(y) \vee \neg R^{\kappa_{2}}(x, z) \vee Q_{\square \neg \psi}(z)$ \\
$5^{\kappa}$ & $\neg \square^{\kappa} \neg \square p \rightarrow \square p$ & $\neg R^{\kappa}(x, y) \vee \neg Q_{\square p}(y) \vee Q_{\square p}(x)$ \\
\hline
\end{tabular}

Fig. 3. Schema clauses $\left(\kappa \geq 1\right.$ and $\left.\kappa_{1}, \kappa_{2} \geq 0\right)$

frame correspondence property (a first-order formula) of some axiom $\mathcal{A} \in \Delta$. Some familiar axioms and their frame correspondence properties are listed in Figure 1. When we use the symbol $\diamond$, as is done in the figure, it is taken to be a shorthand for $\neg \square \neg . R^{0}(s, t)$ denotes $s \approx t$, and for any $n>0, R^{n+1}(s, t)$ denotes $\exists x(R(s, x) \wedge$ $\left.R^{n}(x, t)\right)$.

Theorem 3.2 (Soundness and completeness of $\Pi$ ) Let $\varphi$ be a modal formula, and let $L$ be propositional multi-modal logic $K_{(m)} \Delta$ complete with respect to a class of frames satisfying first-order relational properties. Then $\varphi$ is satisfiable in $L$ iff $\operatorname{Corr}(\Delta) \wedge \Pi(\varphi)$ is satisfiable in first-order logic.

Proof. By the soundness and completeness of the standard translation mapping and the preservation of (un)satisfiability by structural transformation.

For instance, $\varphi$ is satisfiable in $K_{(m)}$ iff $\Pi(\varphi)$ is satisfiable in first-order logic. Also, $\varphi$ is satisfiable in $K 4$ iff $\forall x y z(R(x, y) \wedge R(y, z) \rightarrow R(x, z)) \wedge \Pi(\varphi)$ is satisfiable in first-order logic.

In this paper we assume the clausal form of a first-order formula $\varphi$, written $\mathrm{Cls}(\varphi)$, is computed by transformation into conjunctive normal form, inner Skolemisation, and clausifying the Skolemised formula. Figure 2 lists modal formulae and the clausal form of the corresponding definitional clausal forms.

Lemma 3.3 Let $\varphi$ be a modal formula. Each clause in $\operatorname{Cls} \Pi(\varphi)$ is either (i) the unit clause $Q_{\varphi}(a)$, for some Skolem constant $a$, or (ii) it is an instance of a definitional clause given in Figure 2.

\section{THE AXIOMATIC TRANSLATION PRINCIPLE}

In the axiomatic translation the correspondence properties are replaced by a set of instances of schema clauses. Following the recipe described in Section 2 for the 
axioms 4 and $T$, the schema clauses can be derived fully automatically from the Hilbert axioms. Figure 3 lists the schema clauses of a selection of axiom schemas. The notation $\neg R^{0}(s, t) \vee C$ represents $\neg(s \approx t) \vee C$. If $s$ is a variable that does not occur in $t$ then $\neg R^{0}(s, t) \vee C$ is equivalent to $C\{s / t\}$. For $n \geq 0, \neg R^{n+1}(s, t) \vee C$ represents $\neg R(s, z) \vee \neg R^{n}(z, t) \vee C$ where $z$ is a new variable that does not occur in the clause. The clauses are assumed to be closed under universal quantification of the free variables. The propositional variable $p$ in the schema clauses is a parameter, which will be suitably instantiated in the axiomatic translation defined below.

In this paper we restrict our attention to modal axioms in one variable which are represented by one schema clause. In general, modal axioms may reduce to a set of schema clauses (even for modal axioms in one variable). The principle described in this paper applies uniformly to modal axiom schemas in more than one variable as well.

Now we formalise the principle of the axiomatic translation exemplified in Section 2. Let $L$ be a normal propositional modal logic. That is, $L$ is an extension $K_{(m)} \Delta$ of multi-modal logic $K_{(m)}$, where $\Delta$ denotes a set of axiom schemas. To each axiom $\mathcal{A} \in \Delta$ we assign a (predetermined) instantiation set $\mathfrak{X}_{\mathcal{A}}$. Since the instantiation sets are allowed to vary for different axioms and different logics we let $\mathfrak{X}=\left\{\mathfrak{X}_{\mathcal{A}}\right\}_{\mathcal{A} \in \Delta}$ be a collection of these instantiation sets. Each $\mathfrak{X}_{\mathcal{A}}$ is the instantiation set for an axiom $\mathcal{A}$ in $\Delta$, and $\mathfrak{X}$ is the collection of instantiation sets used in the axiomatic translation of a problem in $K_{(m)} \Delta$. Suitable instantiation sets are specified in the next section when we consider particular modal logics. The collection $\mathfrak{X}$ is always relative to the set $\Delta$ of axiom schemas, but to avoid cluttering we do not make this explicit in the notation.

Definition 4.1 (Axiomatic translation $\Pi_{\mathfrak{X}}^{\Delta}$ ) Let $\Pi_{\mathfrak{X}}^{\Delta}$ be a function mapping modal formulae to first-order formulae defined as follows. If $\varphi$ is an $L$-formula then $\Pi_{\mathfrak{X}}^{\Delta}(\varphi)$ is (the simplification with Simpl of) the conjunction of the following.

(1) The structural translation of $\varphi$.

$$
\Pi(\varphi)=\exists x Q_{\varphi}(x) \wedge \operatorname{Simpl}(\bigwedge\{\operatorname{Def}(\psi) \mid \psi \in \operatorname{Sf}(\varphi)\})
$$

(2) For each axiom schema $\mathcal{A}$ in $\Delta$ and each schema clause $C$ associated with $\mathcal{A}$, the $\psi$ instances of $C$ for each formula $\psi$ in $\mathfrak{X}_{\mathcal{A}}$.

$$
\bigwedge\left\{\operatorname{Ax}^{\mathcal{A}}(\psi) \mid \mathcal{A} \in \Delta, \psi \in \mathfrak{X}_{\mathcal{A}}\right\}
$$

By definition, $\operatorname{Ax}^{\mathcal{A}}(\psi)$ is the conjunction of (the universal closure of) all clauses $C\{p / \psi\}$, where $C$ is a schema clause in the schema clause set associated with the axiom schema $\mathcal{A}$.

(3) The structural translation of subformulae in $X=\left\{\operatorname{Ind}^{\mathcal{A}}(\psi) \mid \mathcal{A} \in \Delta, \psi \in \mathfrak{X}_{\mathcal{A}}\right\}$, where $\operatorname{Ind}^{\mathcal{A}}(\psi)$ denotes the set of modal formulae occurring in the indices of the instances of the clauses associated with the axiom $\mathcal{A}$ as determined by the instance set $\mathfrak{X}_{\mathcal{A}}$.

$$
\operatorname{Simpl}(\bigwedge\{\operatorname{Def}(\psi) \mid \psi \in \operatorname{Sf}(X)\})
$$

ACM Transactions on Computational Logic, Vol. X, No. Y, August 2005. 
(4) The reverse implications of the links between $Q_{\psi}$ and $Q_{\sim \psi}$ in the definitions.

$$
\bigwedge\left\{\forall x\left(\neg Q_{\sim \psi}(x) \rightarrow Q_{\psi}(x)\right) \mid \psi \in \operatorname{Sf}(X) \cup \operatorname{Sf}(\varphi)\right\}
$$

The implicit limitation in the definition to axiom schemas with one free variable, or clauses with one parameter, is not crucial. The definition can be easily generalised for modal axioms in more than one variable.

Notice that the clausal form of the formulae in (4) of Definition 4.1 are positive clauses. As a consequence hyperresolution generates also non-ground conclusions for the axiomatic encoding. They are omitted from the definitions in $\Pi(\varphi)$ so that inferences with non-ground positive premises using the standard translation approach need not be simulated in the completeness proofs. We call the formulae in Definition 4.1.(4) positive shortcut formulae and their clausal form positive shortcut clauses. In general, by shortcut formulae we mean formulae logically equivalent to $\forall x\left(\neg Q_{\sim \psi}(x) \leftrightarrow Q_{\psi}(x)\right)$. Their clausal form are called shortcut clauses and consist of two clauses, a positive and a negative clause. We call them positive and negative shortcut clause, respectively. For some modal logics and modal axioms the positive shortcut formulae are not strictly necessary, however they are included for reasons of uniformity. Experience shows that the presence of the corresponding shortcut clauses can be beneficial, because theorem provers can terminate earlier and the proofs can be shorter.

The definition of the translation mapping contains unnecessarily repeated definitional forms. Since for an arbitrary modal formula $\psi, \operatorname{Def}(\psi)$ and $\operatorname{Def}(\sim \psi)$ are equivalent in the definition of $\Pi_{\mathfrak{X}}^{\Delta}(\varphi)$ it would have been more economic to include definitions and shortcut formulae for unnegated subformulae only. If $\psi$ is a propositional literal then $\operatorname{Def}(\psi)$ can be defined equivalently and more economically by $\forall x\left(Q_{\psi}(x) \rightarrow \pi(\psi, x)\right) \wedge \forall x\left(Q_{\sim \psi}(x) \rightarrow \pi(\sim \psi, x)\right)$. Another possible improvement which is achievable with minimal extra computational overhead replaces the set in (3) of Definition 4.1 by the smaller set

$$
\operatorname{Simpl}(\bigwedge\{\operatorname{Def}(\psi)) \mid \psi \in X \backslash \operatorname{Sf}(\varphi)\}),
$$

which does not include the structural translation of subformulae already in $\Pi(\varphi)$.

Soundness of the axiomatic translation is not difficult to prove. (The formal proof is given in Appendix C.)

Theorem 4.2 (Soundness of $\Pi_{\mathfrak{X}}^{\Delta}$ ) Let $L$ be a propositional modal logic $K_{(m)} \Delta$ with $\Delta$ a (finite) set of modal formulae. Let $\varphi$ be any $L$-formula and assume $\bigcup_{\mathcal{A} \in \Delta} \mathfrak{X}_{\mathcal{A}}$ is a (finite) set of $L$-formulae. If $\varphi$ is $L$-satisfiable then $\Pi_{\mathfrak{X}}^{\Delta}(\varphi)$ is firstorder satisfiable, where $\mathfrak{X}=\left\{\mathfrak{X}_{\mathcal{A}}\right\}_{\mathcal{A} \in \Delta}$.

Basically the proof uses an argument that is standard for proving the soundness of renaming techniques. The only difference is that we start with a modal model and construct a first-order model of the formula $\Pi_{\mathfrak{X}}^{\Delta}(\varphi)$. This model is a conservative extension of the first-order model normally associated with the modal model. An instance of a schema clause is satisfied in the first-order model, since the corresponding instance of the corresponding modal axiom is satisfied in every world of 
the modal model, for, a schema clause is the clausal normal form of a formula obtained by a partial rewriting of an axiom $\mathcal{A}$ with respect to Ohlbach's equivalence preserving rewrite rules.

The soundness result is very general and very useful, especially when considering the following equivalent formulation: If $\Pi_{\mathfrak{X}}(\varphi)$ is unsatisfiable in first-order logic then $\varphi$ is unsatisfiable in $L$. As a consequence, it is possible to use the axiomatic translation method even for proving theorems in non-first-order definable modal logics. For example, we can prove that $D$ is a theorem in $K M$, where $M$ is McKinsey's axiom $\square \diamond p \rightarrow \diamond \square p$. The axiom $M$ reduces to a set of schema clauses and a proof can be found using the $\square$ subformula instances of the schema clauses.

We are also going to show that the classical translation and the axiomatic translation can be used in combination. We refer to such mixed forms of encodings as mixed (axiomatic) reductions or mixed (axiomatic) translations. Here is a general soundness result.

Theorem 4.3 (Soundness of mixed axiomatic reductions) Let $L$ be a propositional modal $\operatorname{logic} K_{(m)} \Delta$ with $\Delta$ a finite set of modal formulae. Suppose $\Delta$ can be partitioned into two sets of axioms, $\Delta^{\prime}$ and $\Delta^{\prime \prime}$, so that all axioms in $\Delta^{\prime}$ are first-order definable. Assume $\bigcup_{\mathcal{A} \in \Delta^{\prime \prime}} \mathfrak{X}_{\mathcal{A}}$ is a finite set of $L$-formulae. Let $\mathfrak{X}=\left\{\mathfrak{X}_{\mathcal{A}}\right\}_{\mathcal{A} \in \Delta^{\prime \prime}}$ and let $\varphi$ be any $L$-formula. If $\varphi$ is $L$-satisfiable then $\operatorname{Corr}\left(\Delta^{\prime}\right) \wedge$ $\Pi_{\mathfrak{X}}^{\Delta^{\prime \prime}}(\varphi)$ is first-order satisfiable.

\section{COMPLETENESS}

In order to prove completeness of the translation mapping $\Pi_{\mathfrak{X}}^{\Delta}$, a possible approach is to take a model-theoretic route and to show how to construct an $L$-model for $\varphi$ from a given first-order model for $\Pi_{\mathfrak{X}}^{\Delta}(\varphi)$, for a predetermined, finitely bounded instantiation set $\mathfrak{X}$. For first-order definable logics it is also sufficient to construct a first-order model for the classical translation of $\varphi$. Alternatively, and this is the approach taken in this paper, one can use a proof-theoretic argument. A prooftheoretic argument sufficient for obtaining completeness is to show how to map refutation proofs of $\varphi$ in $L$ to refutation proofs of $\Pi_{\mathfrak{X}}^{\Delta}(\varphi)$ in first-order logic for a predetermined, finitely bounded $\mathfrak{X}$. Because the standard translation mapping $\Pi$ is sound and complete, we show that a certain style of proofs of the unsatisfiability of $\operatorname{Corr}(\Delta) \wedge \Pi(\varphi)$ using the classical translation approach can be mapped to a proof of the unsatisfiability of $\Pi_{\mathfrak{X}}^{\Delta}(\varphi)$ using the axiomatic translation approach in first-order logic. For various reasons we use hyperresolution style derivations combined with splitting for this purpose. Conclusions with hyperresolution are always positive clauses and, more importantly, the clausal form of $\operatorname{Corr}(\Delta) \wedge \Pi(\varphi)$ for the logics we consider are sets of near range-restricted clauses. A clause $C$ is said to be range-restricted iff the set of variables in the positive part of $C$ is a subset of the set of variables of the negative part of $C$. Derivations with hyperresolution and splitting on range-restricted clauses have the property, that all positive premises of an inference step are ground unit clauses and conclusions are then always positive ground clauses (cf. e.g. [Bry and Yahya 2000; Georgieva et al. 2001]). For the logics we consider clause sets may also contain non-ground positive clauses of the form

ACM Transactions on Computational Logic, Vol. X, No. Y, August 2005. 
( $l$ denotes a natural number): ${ }^{1}$

$$
R(x, x), \quad R(x, f(x)), \quad R(f(x), x) \quad \text { and } \quad R\left(x, f^{l}(x)\right) .
$$

Derivations with hyperresolution and splitting on such near range-restricted clauses have the following property. All positive premises and all (maximally factored and split) conclusions of inference steps are either ground unit clauses or have the form (8). Hyperresolution and splitting not only detects unsatisfiability; for the kinds of problems we consider, in the case that $\varphi$ is satisfiable, a (Herbrand) model can be immediately read off from any complete open branch in a derivation. Moreover, the derivations can be mapped directly to derivations in first-order sentence tableau and in fact also to derivations in labelled modal tableaux.

In proving completeness, a key problem is to determine how many and which instances of the clausal schemas are needed for the axiomatic translation to work. That is, we need to specify the set $\mathfrak{X}$ of formulae for which instances of the schema clauses are to be formed. To do so in a systematic way, we need some more notation. If $X$ is a set of modal formulae then $\square X$ denotes the set $\{\square \psi \mid \psi \in X\}$. Let $\varphi$ be an arbitrary modal formula. We assume that all axiom schemas are unary, i.e. any axiom schema contains only occurrences of one propositional variable. Suppose $\mathcal{A}$ denotes an arbitrary unary axiom schema with free variable $p$, and $\alpha$ denotes a sequence of unary axiom schemas (without repetition). The empty sequence is denoted by $\epsilon$. Now, define $\mathfrak{X}_{\varphi}^{\alpha}$ inductively by: ${ }^{2}$

$$
\begin{aligned}
\mathfrak{X}_{\varphi}^{\epsilon} & =\{\psi \mid \square \psi \in \operatorname{Sf}(\varphi)\} \quad \text { and } \\
\mathfrak{X}_{\varphi}^{\alpha . \mathcal{A}} & =\mathfrak{X}_{\varphi}^{\alpha} \cup\left\{\phi\{p / \psi\} \mid \square \phi \in \square \mathfrak{X}_{\mathcal{A}}^{\epsilon}, \square \psi \in \operatorname{Sf}\left(\square \mathfrak{X}_{\varphi}^{\alpha}\right)\right\}
\end{aligned}
$$

$\mathfrak{X}_{\varphi}^{\epsilon}$ is the set of subformulae of $\varphi$ that occur immediately below a $\square$ operator. In general, $\mathfrak{X}_{\varphi}^{\alpha}$ is the set of subformulae occuring immediately below a $\square$ operator in $\varphi$ and formulae induced by $\square$ subformulae in the axioms in the sequence $\alpha$. Note that the order of the appearance of the axioms in this sequence is essential.

In the remainder of the section we present completeness theorems of the axiomatic translation for a selection of modal logics. All proofs can be found in Appendices D to $\mathrm{F}$.

The axiomatic translation of any formula $\varphi$ in $K 4, K T, K D, K B$, Kalt $_{1}$ is determined by $\mathfrak{X}_{4}=\mathfrak{X}_{T}=\mathfrak{X}_{B}=\mathfrak{X}_{\text {alt }}=\mathfrak{X}_{\varphi}^{\epsilon}$. That is, $\square \mathfrak{X}_{\mathcal{A}}$ is the set of $\square$ subformulae of $\varphi$ for $\mathcal{A} \in\{4, T, D, B$, alt 1$\}$.

Theorem 5.1 Suppose $\mathcal{A} \in\left\{T, B, D, 4\right.$, alt $\left._{1}\right\}$. Let $\varphi$ be any modal formula and assume $\mathfrak{X}=\left\{\mathfrak{X}_{\mathcal{A}}\right\}$ where $\mathfrak{X}_{\mathcal{A}}=\mathfrak{X}_{\varphi}^{\epsilon}$. Then, $\Pi_{\mathfrak{X}}^{\mathcal{A}}(\varphi)$ is unsatisfiable in first-order logic, whenever $\varphi$ is unsatisfiable in $K \mathcal{A}$. The positive shortcut clauses are optional for completeness.

For each of the axioms the structure of the argument is always the same, but in detail the proofs can differ significantly depending on the nature of the modal axiom.

${ }^{1}$ The clauses $R(x, x)$ and $R(x, f(x))$ represent reflexivity and seriality. See the proofs in appendix for the origin of the clauses $R(f(x), x)$ and $R\left(x, f^{l}(x)\right)$.

${ }^{2}$ In general, for multi-modal logics the definition needs to be more complex. 
We can show completeness for the generalised axiom schemas $4^{\kappa}(\kappa \geq 1)$ and alt $\kappa_{1}^{\kappa_{1}, \kappa_{2}}\left(\kappa_{1}, \kappa_{2} \geq 0\right)$, cf. Figures 1 and 3 , and $S_{4}$ using $\mathfrak{X}_{\varphi}^{\epsilon}$.

Theorem 5.2 Suppose $\mathcal{A} \in\left\{4^{\kappa}, a l t_{1}^{\kappa_{1}, \kappa_{2}}\right\}$. Let $\varphi$ be any modal formula and assume $\mathfrak{X}=\left\{\mathfrak{X}_{\mathcal{A}}\right\}$ where $\mathfrak{X}_{\mathcal{A}}=\mathfrak{X}_{\varphi}^{\epsilon}$. If $\varphi$ is unsatisfiable in $K \mathcal{A}$ then $\Pi_{\mathfrak{X}}^{\mathcal{A}}(\varphi)$ is unsatisfiable in first-order logic. The positive shortcut clauses are optional for completeness.

Theorem 5.3 Let $\varphi$ be any modal formula and assume $\mathfrak{X}=\left\{\mathfrak{X}_{T}, \mathfrak{X}_{4}\right\}$, where $\mathfrak{X}_{T}=\mathfrak{X}_{4}=\mathfrak{X}_{\varphi}^{\epsilon}$. Then, $\Pi_{\mathfrak{X}}^{T, 4}(\varphi)$ is unsatisfiable in first-order logic, whenever $\varphi$ is unsatisfiable in $S_{4}$. The positive shortcut clauses are optional for completeness.

Similarly, one can prove that the axiomatic translation using $\mathfrak{X}_{\varphi}^{\epsilon}$ (with or without positive shortcut clauses) for each axiom is complete for $K T B, K D B$ and $K D 4$.

From these results, one might be tempted to think that the axiomatic translation provides a generic and modular approach for translating problem specifications into first-order logic. Unfortunately, in general the solution is not as smooth as above. In general, it is not enough to form one instance of the clausal schemas of an axiom for each $\square$ subformula. It might not even be only $\square$ subformulae instances that are required.

A logic of the former kind is $K 5$. For $K 5$, clausal schema instances need to be formed not only for $\square$ subformulae, but also for $\square \neg \square \psi$ formulae, where $\square \psi$ is a subformula of the candidate formula. A counter example that the axiomatic translation is not always complete for $\square$ subformula instances alone is the formula $\varphi=\diamond \diamond \diamond \neg q \wedge \square \square q$ which is unsatisfiable in $K 5$, but $\Pi_{\mathfrak{X}}^{5}(\varphi)$, where $\mathfrak{X}=\left\{\mathfrak{X}_{\varphi}^{\epsilon}\right\}$, is satisfiable. However, the translation $\Pi_{\mathfrak{X}}^{5}(\varphi)$ can be refuted if $\mathfrak{X}=\left\{\mathfrak{X}_{\varphi}^{5}\right\}$, where $\square \mathfrak{X}_{\varphi}^{5}=\square \mathfrak{X}_{\varphi}^{\epsilon} \cup \square \neg \square \mathfrak{X}_{\varphi}^{\epsilon}$ and hence $\mathfrak{X}_{\varphi}^{5}=\mathfrak{X}_{\varphi}^{\epsilon} \cup \neg \square \mathfrak{X}_{\varphi}^{\epsilon}$.

Theorem 5.4 Let $\varphi$ be any modal formula and let $\mathfrak{X}=\left\{\mathfrak{X}_{5}\right\}$, where $\mathfrak{X}_{5}=\mathfrak{X}_{\varphi}^{5}$. Then, $\Pi_{\mathfrak{X}}^{5}(\varphi)$ is unsatisfiable in first-order logic, whenever $\varphi$ is unsatisfiable in $K 5$.

The theorem is a consequence of a more general result.

Theorem 5.5 Let $\varphi$ be any modal formula and assume $\mathfrak{X}=\left\{\mathfrak{X}_{5^{\kappa}}\right\}$, where $\mathfrak{X}_{5^{\kappa}}=$ $\mathfrak{X}_{\varphi}^{5^{\kappa}}$. If $\varphi$ is unsatisfiable in $K 5^{\kappa}$ then $\Pi_{\mathfrak{X}}^{5^{\kappa}}(\varphi)$ is unsatisfiable in first-order logic.

Theorem 5.3 for $S_{4}$ might lead one to speculate that the principle may be easily extended to $K_{(m)} \Delta$ where $\Delta$ contains multiple axiom schemas. But there are counter examples. While for the logics $K B$ and $K 4$, it is enough to include $\square$ subformula instances of the schema clauses, for the logic $K 4 B$ it is not enough to instantiate the schema clauses for 4 and $B$ with $\square$ subformulae. For example, $5=\neg \square \neg \square p \rightarrow \square p$ is a theorem in $K 4 B$. But $\Pi_{\mathfrak{X}}^{4, B}(\neg 5)$ is not refutable with $\mathfrak{X}=\left\{\mathfrak{X}_{4}, \mathfrak{X}_{B}\right\}$ where $\mathfrak{X}_{4}=\mathfrak{X}_{B}=\mathfrak{X}_{\varphi}^{\epsilon}$, nor with $\mathfrak{X}_{4}=\mathfrak{X}_{\varphi}^{B}$ and $\mathfrak{X}_{B}=\mathfrak{X}_{\varphi}^{\epsilon}$. A refutation proof can be found if $\mathfrak{X}_{4}=\mathfrak{X}_{\varphi}^{\epsilon}$ and $\mathfrak{X}_{B}=\mathfrak{X}_{\varphi}^{4}$.

Theorem 5.6 Let $\varphi$ be any modal formula and assume $\mathfrak{X}=\left\{\mathfrak{X}_{B}, \mathfrak{X}_{4}\right\}$, where $\mathfrak{X}_{4}=\mathfrak{X}_{\varphi}^{\epsilon}$ and $\mathfrak{X}_{B}=\mathfrak{X}_{\varphi}^{4}$. Then, $\Pi_{\mathfrak{X}}^{4, B}(\varphi)$ is unsatisfiable in first-order logic, whenever $\varphi$ is unsatisfiable in $K_{4} B$.

ACM Transactions on Computational Logic, Vol. X, No. Y, August 2005. 
Theorem 5.7 Let $\varphi$ be any modal formula and assume $\mathfrak{X}=\left\{\mathfrak{X}_{T}, \mathfrak{X}_{B}, \mathfrak{X}_{4}\right\}$, where $\mathfrak{X}_{T}=\mathfrak{X}_{4}=\mathfrak{X}_{\varphi}^{\epsilon}$ and $\mathfrak{X}_{B}=\mathfrak{X}_{\varphi}^{4}$. Then, $\Pi_{\mathfrak{X}}^{T, 4, B}(\varphi)$ is unsatisfiable in first-order logic, whenever $\varphi$ is unsatisfiable in $S 5$.

Thus we can summarise:

Theorem 5.8 (Completeness) For each of the modal logics $K \Delta$ considered in this section $\left(K 4, K T, K D, K B\right.$, Kalt $_{1}, K_{4}^{\kappa}, K_{a l t}{ }_{1}^{\kappa_{1}, \kappa_{2}}, K T 4, K T B, K D B, K D 4, K 5$, $\left.K 5^{\kappa}, K 4 B, K T 4 B\right)$ and any modal formula $\varphi$, there is an effectively computable set $\mathfrak{X}$ such that (i) $\varphi$ is satisfiable in $K \Delta$ iff $\Pi_{\mathfrak{X}}^{\Delta}(\varphi)$ is first-order satisfiable. Moreover, (ii) $\Pi_{\mathfrak{X}}^{\Delta}(\varphi)$ can be computed in linear time.

Proof. (i) follows from Theorem 4.2 and the results of this section. (ii) is evident from Lemma 3.1 and Definition 4.1.

While the problems with $K 5$ and $K 4 B$ may give a negative impression of the generality of the axiomatic translation principle, one should note that they correspond exactly to the problems one encounters in the development of tableau calculi for these logics (which use propagation rules). Different from tableaux however the axiomatic translation principle allows for a systematic approach to solving these problems by explicit considerations of instance sets.

Finally, we give some completeness results for mixed axiomatic translations.

Theorem 5.9 (Completeness of mixed reduction for $\boldsymbol{S 5}$ ) Let $\varphi$ be a modal formula and $\mathfrak{X}=\left\{\mathfrak{X}_{4}\right\}$, where $\mathfrak{X}_{4}=\mathfrak{X}_{\varphi}^{\epsilon}$. Then, $\varphi$ is satisfiable in $S 5$ iff $\operatorname{Corr}(\{T, B\}) \wedge$ $\Pi_{\mathfrak{X}}^{4}(\varphi)$ is satisfiable in first-order logic. The positive shortcut clauses are optional for completeness.

Theorem 5.10 (Completeness of mixed reductions for $K D B, K D 4$ ) Let $\varphi$ be a modal formula and assume $\mathfrak{X}=\left\{\mathfrak{X}_{\mathcal{A}}\right\}$, where $\mathfrak{X}_{\mathcal{A}}=\mathfrak{X}_{\varphi}^{\epsilon}$ and $\mathcal{A} \in\{B, 4\}$. Then, $\varphi$ is satisfiable in $K D \mathcal{A}$ iff $\operatorname{Corr}(\{D\}) \wedge \Pi_{\mathfrak{X}}^{\mathcal{A}}(\varphi)$ is satisfiable in first-order logic. The positive shortcut clauses are optional for completeness.

In each of these cases the translated problem can be generated in linear time.

\section{DECIDABILITY}

It is not difficult to see that the axiomatic translation reduces modal problems into decidable fragments of first-order logic, including the two-variable guarded fragment $G F^{2}$ and the class $D L^{*}$.

A formula $\varphi$ belongs to $G F$ if any quantified subformula $\psi$ of $\varphi$ has the form $\exists \bar{y}(G(\bar{x}, \bar{y}) \wedge \phi(\bar{y}))$ or $\forall \bar{y}(G(\bar{x}, \bar{y}) \rightarrow \phi(\bar{y}))$. A $G F$ formula belongs to $G F^{2}$ if it can be expressed using only two variables.

The class $D L^{*}$ [De Nivelle et al. 2000] is a variation of the class of DL-clauses, that was introduced in [Hustadt and Schmidt 2000a] with the purpose of handling expressive description logics. W.l.o.g. it is assumed that all clauses are maximally split. A maximally split clause $C$ is a $D L^{*}$-clause iff the following conditions are satisfied. (i) All literals are unary, or binary. (ii) There is no nesting of function symbols. (iii) Every functional term in $C$ contains all the variables of $C$. (iv) Every binary literal (even if it has no functional terms) contains all the variables of $C$. 
Lemma 6.1 (i) The axiomatic translation of any modal formula is equivalent to a $G F^{2}$ formula. (ii) The axiomatic translation of any modal formula can be embedded in the clausal class $D L^{*}$.

Theorem 6.2 (Decidability) Let $L$ be a propositional modal logic $K_{(m)} \Delta$. Then, $L$ is decidable, whenever the following conditions are satisfied. (i) $\Delta$ is finite. (ii) For any $L$-formula $\varphi$, there are effectively computable sets $\mathfrak{X}_{\mathcal{A}}$ for each $\mathcal{A} \in \Delta$ such that if $\Pi_{\mathfrak{X}}^{\Delta}(\varphi)$ is satisfiable in first-order logic then $\varphi$ is satisfiable in $L$, where $\mathfrak{X}=\left\{\mathfrak{X}_{\mathcal{A}}\right\}_{\mathcal{A} \in \Delta}$.

Proof. By the previous lemma, the decidability of $G F^{2}$ and $D L^{*}$, and since for modal logics axiomatic reductions are sound (Theorem 4.2).

Corollary 6.3 The modal logics considered in the previous section and their fusions are decidable.

Proof. By the results of the previous section there are complete axiomatic reductions for the relevant logics, and decidability follows immediately by Theorem 6.2. The generalisation to fusions is not difficult to prove; it can also be concluded from a general preservation theorem for fusions [Kracht and Wolter 1997].

For any non-serial and non-functional modal logic $L$ considered in this paper, it can be inferred from the completeness proofs that the model constructed by hyperresolution for the axiomatic encoding of an $L$-formula $\varphi$ is a tree model (cf. Lemma E.12). This model can be easily mapped into a corresponding modal (tree) model. In general this model is however not a model of the candidate formula $\varphi$, but can be viewed as a skeleton model which can be completed with respect to the appropriate correspondence properties to an $L$-model of $\varphi$.

Theorem 6.4 (Small model property, model generation) Let $L$ be a nonserial and non-functional modal logic considered in the previous section or a fusion of these. If a modal formula $\varphi$ is $L$-satisfiable then (i) it is satisfiable in a model with at most $2^{\mathcal{O}(|\varphi|)}$ elements, i.e. $L$ has the small model property, and (ii) resolution can be used to construct an $L$-model for $\varphi$.

(The proof can be found in Appendix G.) For the logics involving $D$ and $a l t_{1}^{\kappa_{1}, \kappa_{2}}$ the proofs are a bit more involved but we expect the same result to hold for these logics.

Decidability of extensions of $K$ with the axioms $T, B, D, 4,5$, alt $t_{1}$ is well-known. In Gabbay [1975] the decidability of the logics $K 4^{\kappa}, K 5^{\kappa}$ and $K a l t_{1}^{\kappa_{1}, \kappa_{2}}$ was shown by using a reduction to $\mathrm{S} \omega \mathrm{S}$. It is also well-known that the operation of fusing normal propositional modal logics preserves decidability. Thus the decidability of the logics considered is known or follows from known results. As for the small model property, we believe the small model property of the logics $K 4^{\kappa}, K 5^{\kappa}$ and Kalt $_{1}^{\kappa_{1}, \kappa_{2}}$ has been open up to now.

We are however not merely interested in decidability and the small model property. One of our main interests is the development of practical decision procedures. Given a finite axiomatisation and a small model property result, a decision procedure can be defined, but not a very useful one. By contrast since the axiomatic 
translation embeds the modal problems into $D L^{*}$ or $G F^{2}$ and since both can be decided with resolution the following is immediate.

Theorem 6.5 (Decidability by resolution) Resolution based on any refinement compatible with the ordering $>_{d}$ defined in [De Nivelle et al. 2000] decides the axiomatic encoding of satisfiability problems in all modal logics satisfying the conditions of Theorem 6.2.

Most modern first-order logic theorem provers implement ordered resolution and the ordering $>_{d}$ is standardly available in such theorem provers. Consequently, these provers immediately provide practical decision procedures for solving the satisfiability problem of many modal logics. All that needs implementing is the axiomatic translation of modal problems into first-order logic. This is not difficult.

It should be noted that the result is not true for hyperresolution which is used in the completeness proofs. With the blocking inference rule introduced in Hustadt and Schmidt [1999c] hyperresolution can however be turned into a decision procedure for the axiomatic translation of the considered logics.

What about the decidability of reductions using a mix of the standard relational translation and the axiomatic translation? Here we can state the following general theorem.

Theorem 6.6 (Decidability for mixed reduction) Let $L$ be a propositional modal logic $K_{(m)} \Delta$ and suppose $\Delta$ is partitioned into two sets $\Delta^{\prime}$ and $\Delta^{\prime \prime}$. Then, $L$ is decidable, whenever the following conditions are satisfied. (i) $\Delta$ is finite. (ii) For each axiom $\mathcal{A}$ in $\Delta^{\prime}, \operatorname{Corr}(\mathcal{A})$ is expressible in $D L^{*}$. (iii) For any $L$-formula $\varphi$, there are effectively computable sets $\mathfrak{X}_{\mathcal{A}}$ for each axiom $\mathcal{A}$ in $\Delta^{\prime \prime}$ such that, if $\psi=\operatorname{Corr}\left(\Delta^{\prime}\right) \wedge \Pi_{\mathfrak{X}}^{\Delta^{\prime \prime}}(\varphi)$ is satisfiable in first-order logic then $\varphi$ is satisfiable in $L$, where $\mathfrak{X}=\left\{\mathfrak{X}_{\mathcal{A}}\right\}_{\mathcal{A} \in \Delta^{\prime \prime}}$.

Moreover, resolution based on any refinement compatible with the ordering $>_{d}$ decides the satisfiability of $\psi$ in $L$.

Correspondence properties expressible in $D L^{*}$ include the Boolean combination of relational inclusions or equivalences expressed over intersection, union, complementation, converse and positive occurrences of relational composition. For example, the inclusion $R \subseteq R^{\smile}$ expresses the symmetry of $R$, and $R \subseteq R ; R$ expresses a kind of confluence. In addition, reflexivity, irreflexivity and properties such as $\forall x y(R(x, y) \rightarrow R(x, x))$ can be expressed in $D L^{*}$. More details on relational properties expressible in $D L^{*}$ can be found in Schmidt and Hustadt [2003]. Theorem 6.6 is also true for $G F^{2}$. Note however that the logic $D L^{*}$ is more expressive than $G F^{2}$, since $G F^{2}$ can be linearly reduced to $D L^{*}$ using standard structural transformations to clausal form. Since $G F^{2}$ is EXPTIME-complete [Grädel 1999] and $D L^{*}$ is NEXPTIME-complete, it is probable that no linear reduction exists in the other direction. The fact that $D L^{*}$ is NEXPTIME-hard follows since Boolean modal logic is NEXPTIME-complete [Lutz and Sattler 2002] and linearly reduces to $D L^{*}$ [De Nivelle et al. 2000]. It follows from the results of Lewis [1980] that $D L^{*}$ is in NEXPTIME and hence NEXPTIME-complete.

As a consequence of the previous theorem we can state: 
Corollary 6.7 Ordered resolution decides the mixed reductions of the logics KT4, $K D B$ and $K D 4$ defined in Theorems 5.9 and 5.10.

\section{PRACTICAL EXPERIENCE}

The attractiveness of the axiomatic translation principle is its amenability for immediate automation. We have developed a prototype implementation in Prolog of the axiomatic translation. The choice of existing first-order logic provers that can be used is extensive. In our tests we used vampire [Riazanov and Voronkov 1999], DCTP [Letz and Stenz 2001; Stenz 2002], E [Schulz 2002] and (M)SPASs [Hustadt and Schmidt 2000b; Weidenbach et al. 2002]. These are among the best first-order theorem provers currently available. VAMPIRE, E and MSPASS are resolution provers and support ordered resolution refinements compatible with the ordering $>_{d}$, and in fair mode, all provide decision procedures for the axiomatic translation of all modal logics which satisfy the general conditions of Theorems 6.2 and 6.6. DCTP is based on the disconnection calculus. It is not known to be a decision procedure for the problems used in the test, but performed remarkably well in our experiments.

The problems used in the tests were collected during the period of the investigation; a selection are from the literature, but most were created for the purpose of gaining insights into the properties of the axiomatic translation and the simulation of hyperresolutions proofs using different translation methods. As a consequence the majority of the problems are small in size; on average the size of the input files is $4.3 \mathrm{~KB}$ and $4.7-19.8 \mathrm{~KB}$ for the classical translation and axiomatic translation, respectively. In total 81 problems were tested in each of the following logics using both the axiomatic translation and the classical translation. To simplify the subsequent presentation of the results, the logics are numbered as indicated in brackets below.

$$
\begin{aligned}
& K 4 B(0), K 4^{2}(1), K 4^{3}(2), K 4(3), K 5^{2}(4), K 5^{3}(5), K 5(6), K B(7), \\
& K D 4(8), K D B(9), K D T(10), K D(11), K T 4 B(12), K T 4(13), K T B(14), \\
& K T(15), K_{\text {Kalt }}^{1,1}(16), K_{1} \text { Kalt }_{1}^{1,2}(17), \text { Kalt }_{1}^{2,1}(18), K_{1} \text { Kalt }_{1}^{2,2}(19), \text { Kalt }_{1}(20)
\end{aligned}
$$

The graphs in Figures 4 to 7 present a summary of the tests performed with VAMPIRE Version 7.0, E Version 0.82, MSPASS Version 1.0.0t.1.3 and DCTP Versions 10.21 and $1.31(\mathrm{EPR}){ }^{3}$ The tests were run on a Linux PC with a $1.80 \mathrm{GHz}$ Pentium $4 \mathrm{CPU}$ and $512 \mathrm{MB}$ main memory. Each problem was run with a timeout of 200 seconds.

In the first series of graphs (in Figures 4 to 6 ) the number of problems solved within this time serves as the measure of comparison. For each prover the graphs summarise the total number of problems solved and how many of these were satisfiable and unsatisfiable. The first two graphs give information of the performance of the provers in the different logics. The logics are represented by integers 0 to 20 on the $x$-axis in the order as listed above. The additional points reflect the relative increase in the total size of the input files for the axiomatic translation. The third

\footnotetext{
${ }^{3}$ VAMPIRE settings: -t 200, E settings: -xAuto -tAuto -1 0 --tptp-in --resources-info --cpu-limit=202 --memory-limit=192, MSPASS settings: -CNFRenQuant=1 -CNFRenMatch=0

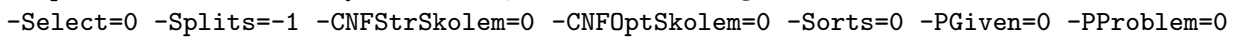
-Timelimit=200, DCTP settings: 210. The executables of VAMPIRE, E, DCTP were obtained from the CASC-2004 Competition website http://www.cs.miami.edu/ ${ }^{\text {tptp/CASC/J2/. }}$
}

ACM Transactions on Computational Logic, Vol. X, No. Y, August 2005. 

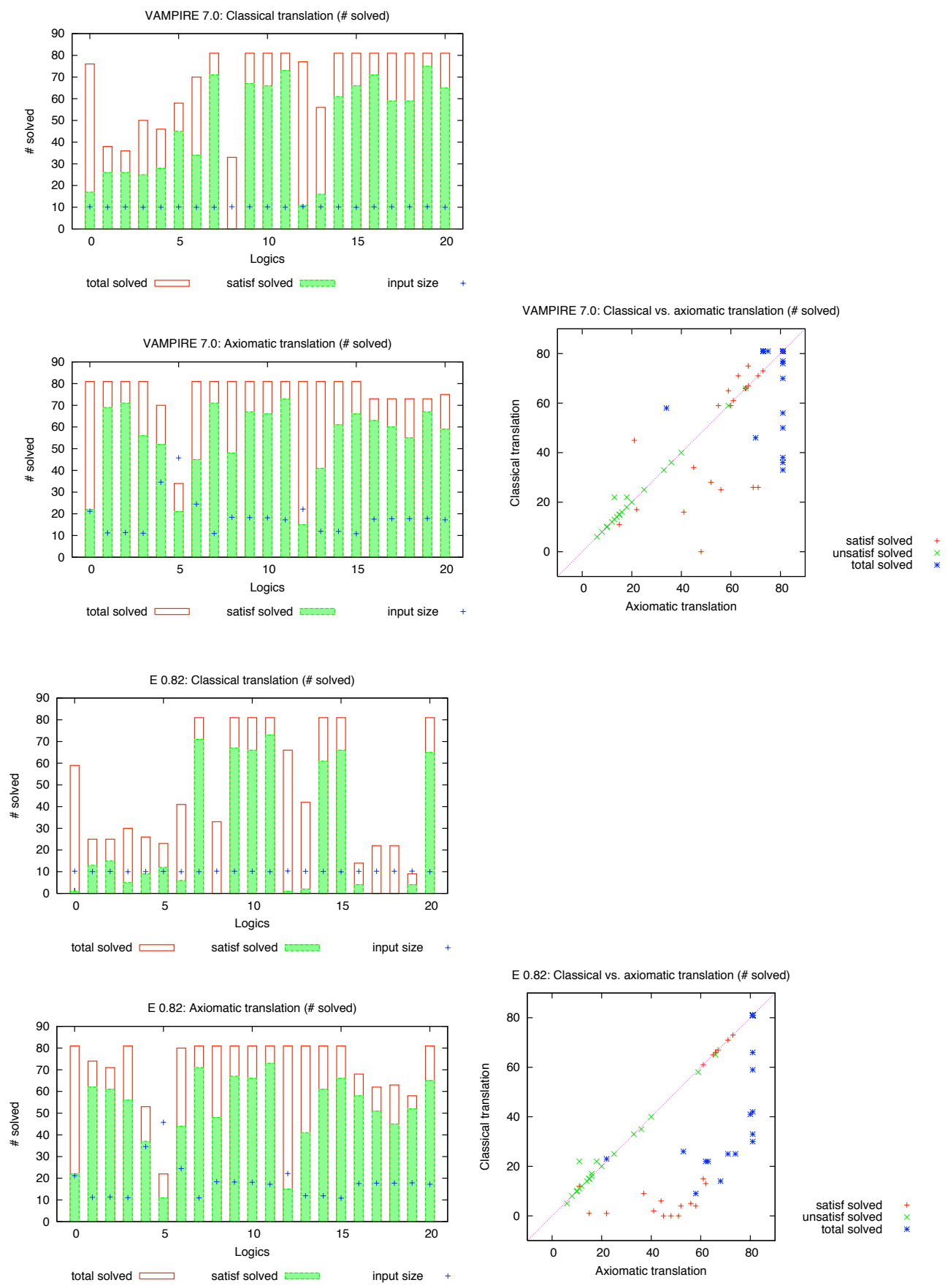

Fig. 4. Number of problems solved: vampiRE and E 

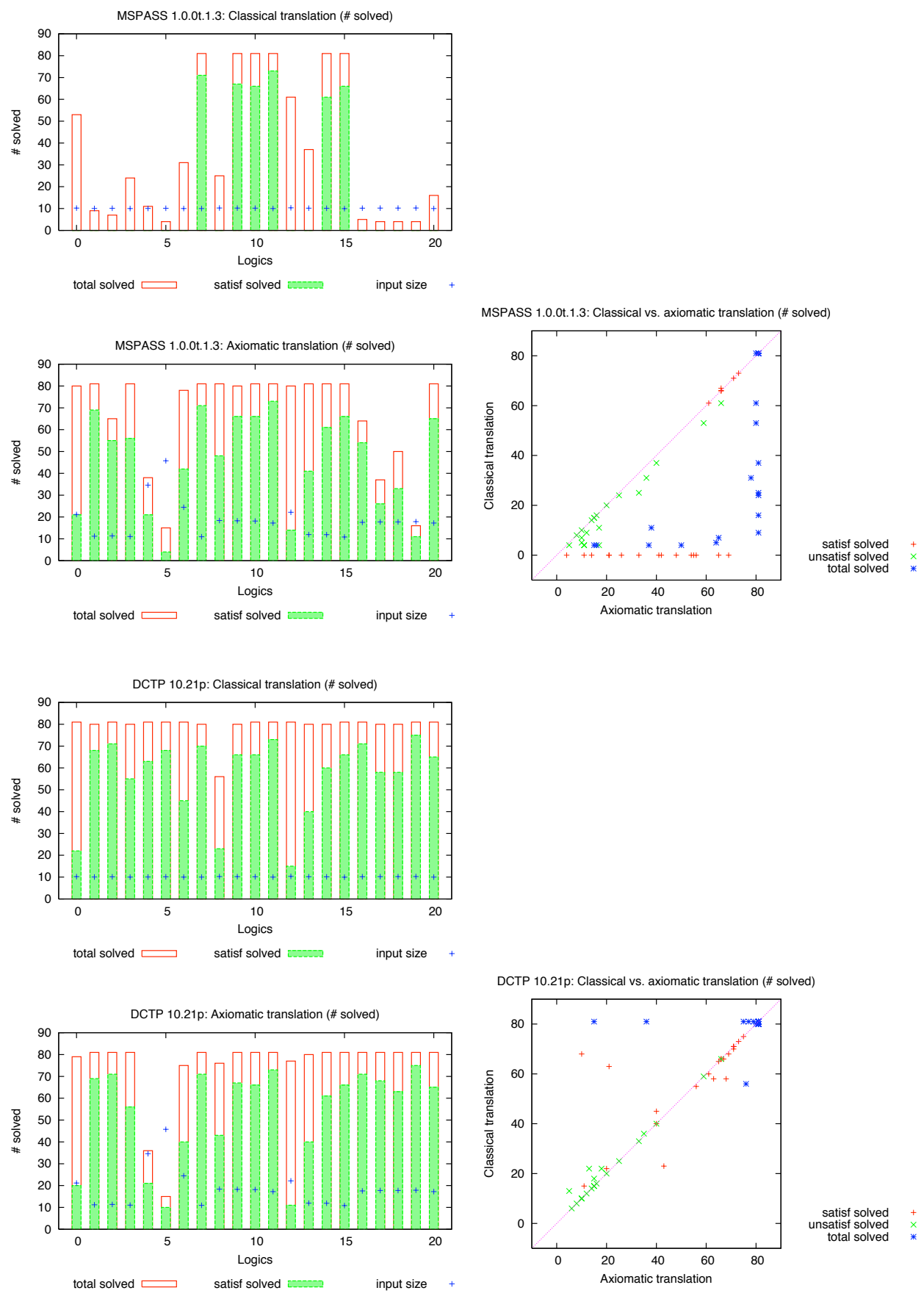

Fig. 5. Number of problems solved: MSPASS and DCTP

ACM Transactions on Computational Logic, Vol. X, No. Y, August 2005. 

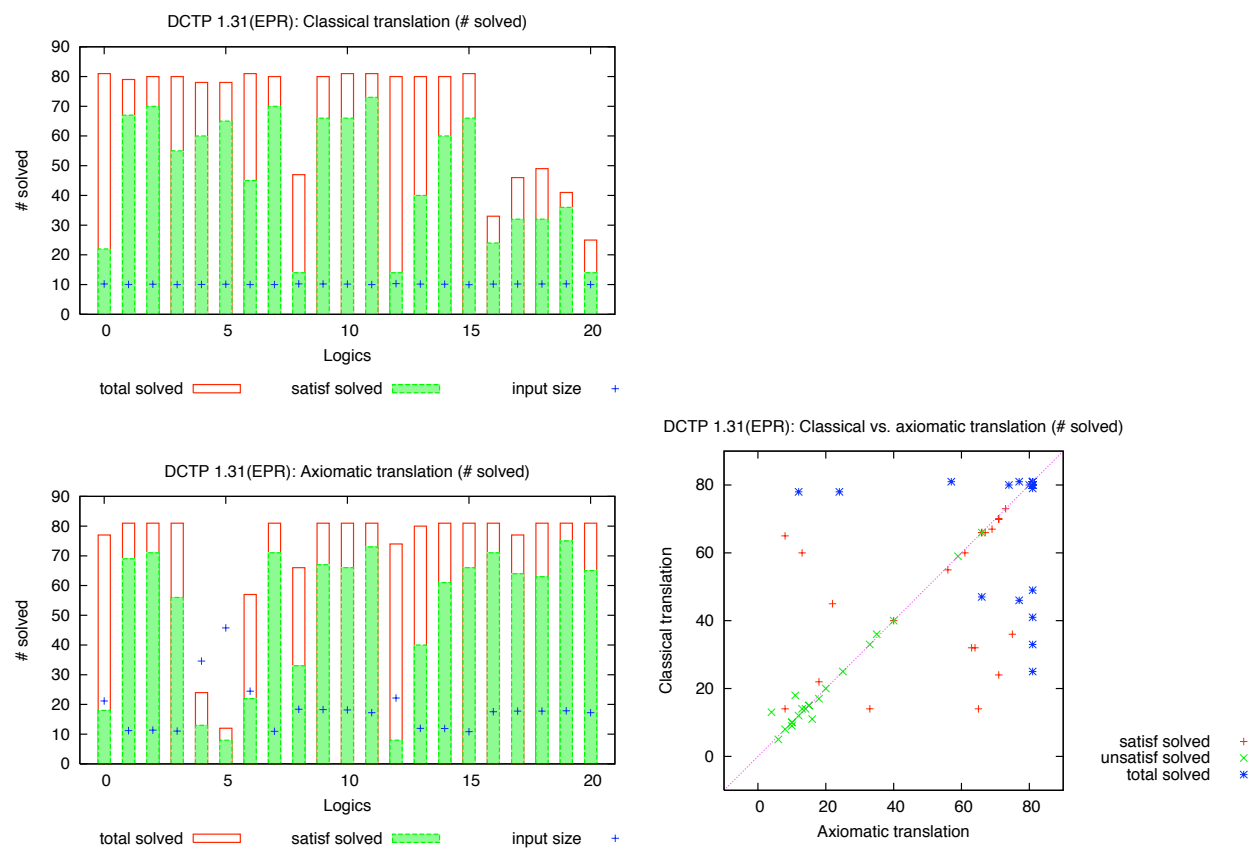

Fig. 6. Number of problems solved: newer version of DCTP

graph (in Figures 4 to 6) provides a direct comparison of the classical and axiomatic translation for all problems. Three points appear in the graph for each of the 21 logics, one for the total number of the 81 problems solved, one for the total number of satisfiable problems solved and one for the total number of unsatisfiable problems solved. Each point at coordinate $(a, c)$ indicates that $a$ number of problems were solved, within the timelimit, using the axiomatic translation method, and $c$ using the classical translation method. More points below the diagonal mean that the performance for the axiomatic translation method in terms of the number of problems solved was better than for the classical translation method (and vice versa if the other way around). In the graphs of Figure 7 the CPU time performance serves as the measure of the comparison.

On the whole the results show that the performance of the resolution provers is better on the axiomatic translation than the classical translation, especially for satisfiable problems and also for logics determined by 'problematic' correspondence properties such as transitivity, Euclideanness and functionality. In particular, on satisfiable problems the provers are performing better on the axiomatic translation. For unsatisfiable problems the picture is less uniform however. Looking at Figures 4 to 6 , for unsatisfiable problems the performance of the provers are roughly on a par for the two translation methods, although interestingly for the faster provers the scale is slightly tipped toward the classical translation method. For the logic $K 5^{3}(5)$ the performance of MSPASS is better for the axiomatic translation, while the performance of $\mathrm{E}$ is the same and the performance of VAMPIRE is better for 

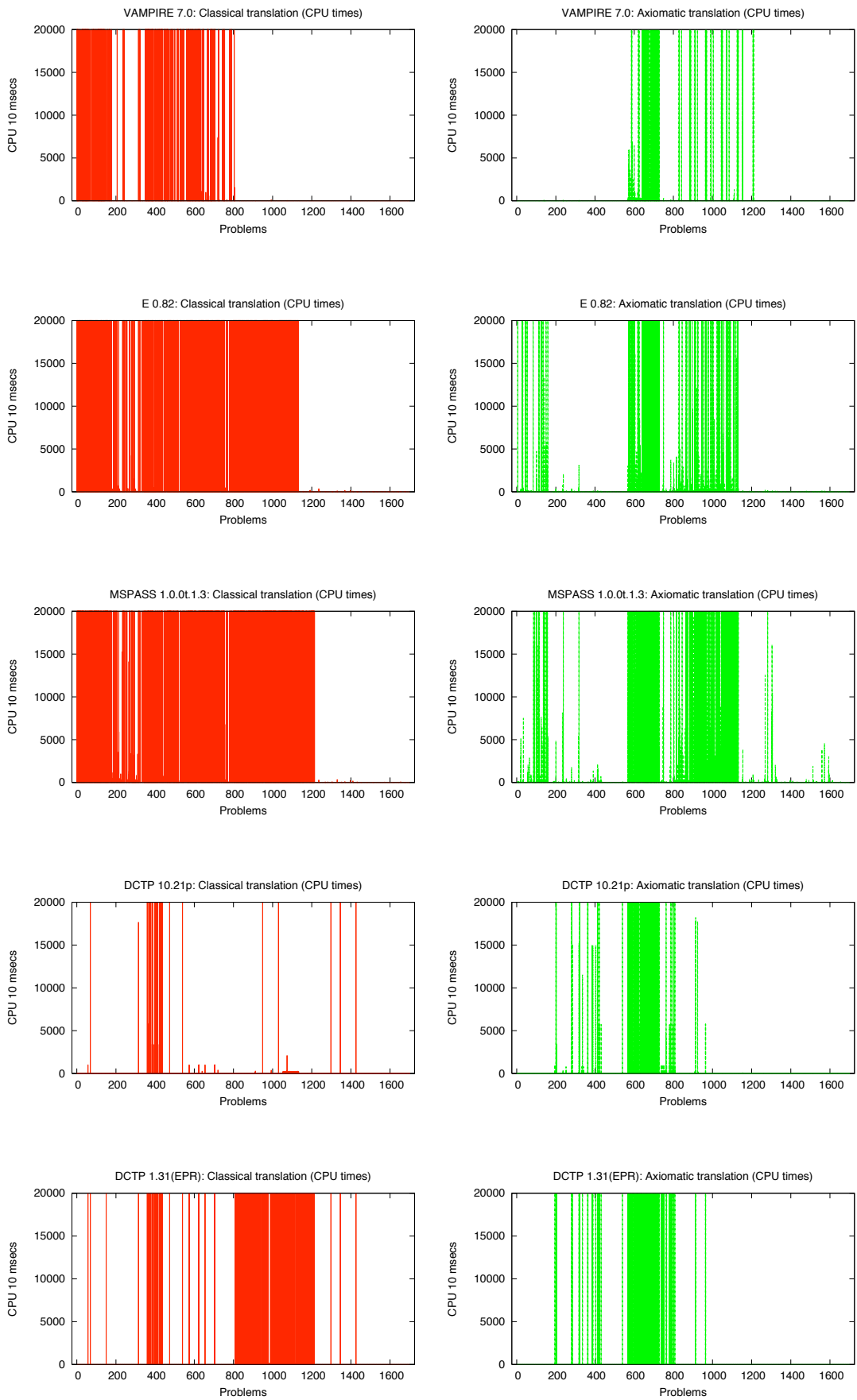

Fig. 7. CPU time results

ACM Transactions on Computational Logic, Vol. X, No. Y, August 2005. 
the classical translation. VAMPIRE's performance on the classical translation is also slightly better for extensions of $K$ involving instances of the alt ${ }_{1}^{\kappa_{1}, \kappa_{2}}$ axiom. The results for $K 5^{3}$ (5) in the left graphs of Figures 4 to 6 suggest that the performance of the provers is sensitive to the size of the translation. Also noteworthy is that for $K 5^{3}$ the instantiation sets are significantly larger than for the other logics.

The tests with the different versions of DCTP showed that the older version, 10.21, performs better than the latest release, 1.31(EPR). This behaviour is consistent with the CASC-2004 theorem prover competition where DCTP 10.21 won the EPR category. The EPR category includes problems encoding local satisfiability in $K$ or $K_{(m)}$ using a variation of the optimised function translation method which maps the problems into the intersection of fluted logic and the Bernays-Schönfinkel class [Hustadt and Schmidt 1999a]. For this translation any resolution or instantiation-based theorem prover is a decision procedure, including DCTP. Since the problems used in the experiments here have different properties to the problems in the EPR category, the outcome of the tests for DCTP are interesting and worth investigating further.

The CPU time results in Figure 7 also show the advantage of the axiomatic translation approach (right graphs) over the classical translation (left graphs) for tests performed. For many of problems actually improvements by factors of 1010000 are observable, and some problems which are not solvable by the classical translation approach have now become solvable with the axiomatic method.

All this is interesting, but clearly, more experiments and a more thorough investigation of the results are needed to get a better understanding for the behaviour in practice of the axiomatic translation method. Future investigations should also include tests with the mixed translation approach.

Another possibility as yet untested is the combination of the axiomatic translation and modal logic or description logic theorem provers. An alternative to using firstorder logic theorem provers, is to use modal logic or description logic theorem provers. It is not difficult to see, as we outline in the next section, that the axiomatic translation can be transferred to the level of modal logic or description logic and combined with a suitable theorem prover for that logic. What is required is either a description logic prover for $\mathcal{A L C}$ that can handle terminological axioms or a modal theorem prover that can handle multi-modal $K_{(m)}$ with non-logical axioms, i.e. global satisfiability (or global validity) in $K_{(m)}$, or a prover for the logic $K_{(m)}$ with the universal modality can be used.

\section{OTHER CONSEQUENCES AND RELATED WORK}

An EXPTIME upper bound for the complexity of the considered logics or their fusions is immediate from the reduction to $\mathrm{GF}^{2}$. This bound is not optimal and can be improved for certain logics. Let $L$ be any of the $\operatorname{logics} K T, K B, K T B$, $K_{a l t} \kappa_{1}^{\kappa_{1}, \kappa_{2}}, K D, K D B$, or their fusions. One can observe that the axiomatic reduction is acyclic in the sense of [Georgieva et al. 2002]. That is, an acyclic dependency relation can be defined over the predicate symbols in the clausal form (obtained by using structural transformation) and can be extended to an ordering $\succ_{D}$ on ground clauses. For any of the logics $L$ this ordering is acyclic and we can show that the axiomatic translation of $L$ belongs to the class $\mathcal{B} U$ of clause sets [Georgieva et al. 2002]. It follows from the investigations of classes decidable by hyperresolution in 
Georgieva et al. [2001; 2002] that:

Theorem 8.1 Let $L$ be any of the logics $K T, K B, K T B, K_{a l t}{ }_{1}^{\kappa_{1}, \kappa_{2}}, K D, K D B$, or their fusions. Then (i) hyperresolution can decide the satisfiability of $\varphi$ in $L$, (ii) hyperresolution can return a Herbrand model which can be transformed into a modal model if $\varphi$ is $L$-satisfiable, and (iii) the minimal model generation procedures described in Georgieva et al. [2001; 2002] can be used to compute all and only minimal Herbrand models, if $\varphi$ is $L$-satisfiable.

It is actually possible to show that the resolution procedure described in Georgieva et al. [2001, §3] decides these logics in polynomial space. In terms of complexity this is an optimal result because a result of Lutz [1999] for the description logic $\mathcal{A L C}$ with acyclic TBoxes implies:

Theorem 8.2 (Complexity) The computational complexity of the satisfiability problem of each of $K T, K B, K T B$, Kalt $_{1}^{\kappa_{1}, \kappa_{2}}, K D, K D B$ and their fusions is PSPACE-complete.

For the other modal logics, for example transitive modal logics, the dependency relationship $\succ_{D}$ is cyclic because of the presence of the same predicate symbol with opposite polarity, for example, in the schema clause for 4 . However, by using the axiomatic translation and the ideas of Kracht [2002] optimal space bounds can be given also for the other logics.

There is a close relationship between hyperresolution and tableau calculi. In Hustadt and Schmidt [2002] and De Nivelle et al. [2000] it is shown how this relationship can be exploited for systematically developing sound, complete and terminating tableau proof systems for PDL-like multi-modal logics. The same approach can be used for extracting tableau rules from the combination of the axiomatic translation and hyperresolution. The idea is to express a group of hyperresolution inference steps as a tableau rule. This technique works readily for modal logics which do not require nested instantiation and where the shortcut clauses of Definition 4.1.(4) are not needed. The tableau calculi obtained are summarised in Figures 8 and 9 . The symbols $s, t, t^{\prime}$ denote constants (or ground terms). ( $\left.K\right)$ represents the rules $(\perp),(\neg),(\wedge),(\vee),(\square),(\neg \square)$. The correspondence between the tableau rules and the schema clauses is evident if one thinks of a schema clause first as an implication and then as a rule. The presented calculi coincide exactly with labelled semantic tableau calculi developed elsewhere, cf. [Goré 1999; Castilho et al. 1997; Massacci 2000]. Thus, the axiomatic translation offers another way of proving the soundness and completeness of these calculi. For the other logics such as $K 5$ and $K 4 B$ where the shortcut clauses are used in the completeness proofs for the axiomatic translation, the tableau calculi obtained in this way include cut rules resulting from the positive shortcut clauses $Q_{\sim \psi}(x) \vee Q_{\psi}(x)$. In all cases the cut rules are analytic, more specifically, they are applied only over the subformulae of the candidate formula and the formulae in $X$ of part (3) of Definition 4.1.

As already noted the axiomatic translation can also be interpreted on the modal level. On the modal level it corresponds to a reduction of the satisfiability of a modal formula with respect to an extension $K_{(m)} \Delta$ of $K_{(m)}$, to global satisfiability

ACM Transactions on Computational Logic, Vol. X, No. Y, August 2005. 


$$
\begin{array}{rrr}
(\perp) \frac{s: \psi, s: \neg \psi}{s: \perp} \quad(\neg) \frac{s: \neg \neg \psi}{s: \psi} & (\wedge) \frac{s: \psi \wedge \phi}{s: \psi, s: \phi} & (\vee) \frac{s: \psi \vee \phi}{s: \psi \mid s: \phi} \\
(\neg \square) & \frac{s: \neg \square \psi}{(s, t): R, t: \neg \psi} \text { where } t \text { is new to the branch } & (\square) \frac{(s, t): R, s: \square \psi}{t: \psi} \\
(T) \frac{s: \square \psi}{s: \psi} & (D) \frac{s: \square \psi}{s: \neg \square \neg \psi} & (B) \frac{(s, t): R, t: \square \psi}{s: \psi} \\
(4) \frac{s: \square \psi,(s, t): R}{t: \square \psi} & \left(\text { alt }_{1}\right) \frac{s: \neg \square \psi}{s: \square \neg \psi} \\
\left(4^{\kappa}\right) \frac{s: \square \psi,(s, t): R^{\kappa}}{t: \square \psi} & \left(\text { alt }_{1}^{\kappa_{1}, \kappa_{2}}\right) \frac{(s, t): R^{\kappa_{1}, t}, t \neg \square \psi,\left(s, t^{\prime}\right): R^{\kappa_{2}}}{t^{\prime}: \square \neg \psi}
\end{array}
$$

Fig. 8. Tableau expansion rules

$\begin{array}{lllll}K: & (K) & K D:(K),(D) & K T: & (K),(T) \\ K B: & (K),(B) & K 4:(K),(4) & \text { Kalt }_{1}: & (K),\left(\text { alt }_{1}\right) \\ K T B:(K),(T),(B) & K D B:(K),(D),(B) & K_{4}: & (K),(D),(4) \\ S_{4}: & (K),(T),(4) & K_{4}{ }^{\kappa}:(K),\left(4^{\kappa}\right) & \text { Kalt }_{1}^{\kappa_{1}, \kappa_{2}}:(K),\left(\text { alt }_{1}^{\kappa_{1}, \kappa_{2}}\right)\end{array}$

Fig. 9. Tableau calculi

in $K_{(m)}$, or satisfiability in $K_{(m)}$ enhanced with the universal modality (which can be further reduced to local satisfiability in $K_{(m)}$ [Goranko and Passy 1992]). On the modal level the axiomatic translation is closely related to the reduction functions introduced independently by Kracht in [1999] and developed further in [2001]. Kracht's reduction functions are defined differently to the ones implicit in the axiomatic translation, the most important difference being that they do not use new symbols for subformulae. Kracht has proved a number of results which immediately carry over to the axiomatic translation. For example, the logics considered in the present paper satisfy Kracht's criteria for local interpolation. A modal logic $L$ has local interpolation, if for every pair $\varphi$ and $\psi$ of formulae with $\varphi \vdash_{L} \psi$, there is a $\chi$ such that $\operatorname{var}(\chi) \subseteq \operatorname{var}(\varphi) \cap \operatorname{var}(\psi)$ and $\varphi \vdash_{L} \chi$ as well as $\chi \vdash_{L} \psi$. We can conclude the following.

Theorem 8.3 (Local interpolation) The modal logics considered in Section 5 and their fusions have local interpolation.

Proof. For each of the axioms $\mathcal{A}, \Pi_{\mathfrak{X}}^{\mathcal{A}}(\neg(\varphi \rightarrow \psi))$ is satisfiable iff $\Pi_{\mathfrak{X}}^{\mathcal{A}}(\varphi) \cup$ $\Pi_{\mathfrak{X}}^{\mathcal{A}}(\neg \psi)$ is satisfiable. Thus the corresponding reduction function on the modal level has the same property. It follows that the Kracht-reduction uses splitting reduction sets, cf. [Kracht 2001]. The result follows then by Theorem 6.8 in [Kracht 2001], since $K$ has local interpolation, and the formulae in $\mathfrak{X}$ are defined over the propositional symbols in the candidate formula. Finally, note that fusion preserves local interpolation [Kracht and Wolter 1997].

The axiomatic translation can be used to combine different styles of reasoning systems. For example, in Hustadt, Dixon et al. [2000] the axiomatic translation was used in a decision procedure for the join of discrete linear temporal logic with 
subsystems of $S 5$. The system combines temporal resolution for the temporal part and first-order resolution on the modal part.

The idea of incorporating a theory into the translation of a formula, or eliminating a relation with special properties by an axiomatic internalisation, is often implicit in reductions between logics. For example, De Giacomo [1996] embeds CPDL into PDL by using an axiomatic encoding of the axiom defining the converse operator. Balbiani and Herzig [1994] and Demri and Goré [1999] respectively embed the provability logics $\mathrm{G}$ and Grz into $\mathrm{GF}^{2}$. Both are second-order modal logics.

There is a (non-obvious) connection to work of Demri and De Nivelle [2003], which is worth exploring further. Demri and De Nivelle show that modal regular grammar logics with converse $R G L(\sim)$ are decidable by reduction to $\mathrm{GF}^{2}$. Demri [2001] has shown that regular grammar modal logics have a number of nice properties. For a given modal logic it is however not immediate whether it is in fact an $R G L(\smile)$ logic. One first has to find a regular expression which describes the closure of the relational correspondence properties of the modal logic. In some instances it can be easily seen that the axiomatic translation is equivalent to the translation of DemriDe Nivelle. In other instances the connection is not immediate. While we have shown how to reduce serial modal logics by our method, no regular expressions are known for relational theories including seriality. In fact, it has been shown by Demri and Orlowska [1999] that seriality and other existential properties of relations are not definable by any closure relation operation.

\section{CONCLUSIONS}

The ideas of the axiomatic translation principle are of interest for various reasons. On the one hand, of interest to pure modal logic are the results obtained and their generality. We have shown general results on decidability, the small model property and the interpolation property and considered some instances of modal logics for which the results hold, including extensions of basic modal logic with lessstudied generalised axiom schemes. Also of interest are the methods used in this paper. Our framework departs significantly from the model-theoretic and algebraic route traditionally followed in modal logic. The paper shows that it is possible to use proof-theoretic methods for proving results traditionally proved using sophisticated model-theoretic constructions (canonical models, filtrations, etc). By using methodologies from resolution and first-order logic we showed how it is possible to directly exploit powerful methods and results available in this framework. A further advantage are the implemented inference tools and theorem provers which are immediately available and allowed us to obtain first, very promising practical experience with only modest implementation effort. In this context the work provides not only new insight into the 'nice' properties of modal logic, but suggests new ways of developing automated reasoning technologies for modal logics and also description logics and other related logics like the guarded fragment.

In this paper our investigation was restricted to local satisfiability problems in modal logics. Most of the results can however be extended to the corresponding global satisfiability problems. This is the case for all soundness, completeness, decidability and small model property results in this paper. The least obvious are of course the completeness proofs, however with the ideas of [Hustadt and Schmidt

ACM Transactions on Computational Logic, Vol. X, No. Y, August 2005. 
1999c] the proofs are only slight modifications of the proofs given in this paper. One can further observe that the global satisfiability problem of all logics which satisfy the conditions of Theorems 6.2 is EXPTIME-complete provided the axiomatic reduction mapping is a polynomial function. For these logics the combination of the axiomatic translation and ordered resolution provides a decision procedure with optimal, EXPTIME complexity. Due to space limitations the formal development of these aspects is left for another paper.

As already indicated in various places this work raises a number of intriguing questions for further research. An important question of general significance to the efficiency of proof methods exploiting the axiomatic translation is whether the size of the axiomatic translation can be improved. The first step is to eliminate duplicate definitions from the axiomatic translation of the problem. In addition, in many instances as indicated in Section 5 it is not difficult to see that the shortcut clauses are redundant and can be omitted, but in some cases they are necessary. Experience shows that shortcut clauses can improve the performance of a prover, so omitting shortcut clauses may not always lead to a performance gain. The impact of smaller instantiation sets is also significant. Are the reductions in this paper optimal with respect to the sizes of the instantiation sets? What is the smallest set of instances necessary for the axiomatic translation of a given logic? Is there a reduction of CPDL to PDL which uses smaller instantiation sets than the Fischer-Ladner closure? These are important questions which deserve further investigation. Another question one might ask is: Are all modal logics effectively reducible to $\mathrm{GF}^{2}$ using the principles of the axiomatic translation (or, equivalently, are all modal logics effectively reducible to the basic modal logic $\left.K_{(m)}\right)$ ? The answer to this questions is clearly no, because there are undecidable extensions of $K_{(m)}$, cf. e.g. [Kracht 1995]. Thus the question should be qualified: Are all decidable modal logics effectively reducible to $\mathrm{GF}^{2}$, or $K_{(m)}$ ? Are there decidable modal logics which cannot be reduced to $\mathrm{GF}^{2}$ or $K_{(m)}$ by ideas based on the axiomatic translation principle?

\section{APPENDIX}

\section{A. BASICS OF MODAL LOGIC}

The purpose of this section is to review briefly the basic notions of propositional modal logics. Standard references include [Blackburn et al. 2001; Chellas 1980; Goldblatt 1987; Hughes and Cresswell 1996].

For reasons of simplicity this paper considers only uni-modal logics. The language is that of propositional logic plus one unary modal operator $\square$ (the box operator). Let $V$ be a finite set of propositional variables $p, q, r, \ldots$. A modal formula is either a propositional atom, i.e. a propositional variable, $\perp$, or a formula of the form $\neg \psi, \psi \wedge \phi$ and $\square \psi$, where $\psi$ and $\phi$ denote modal formulae. $\top, \vee, \rightarrow, \leftrightarrow$ and $\diamond$ are defined as expected. A modal axiom schema is a modal formula representing the collection of all its instances. A modal axiom is an instance of a modal axiom schema.

A (normal) modal logic is defined by a set of modal formulae which includes all propositional tautologies over some modal language and the schema

$K \quad \square(p \rightarrow q) \rightarrow(\square p \rightarrow \square q)$,

and which is closed under modus ponens and the rule of necessitation: 
$M P$ if $\vdash p$ and $\vdash q$ then $\vdash p \rightarrow q$

$N \quad$ if $\vdash p$ then $\vdash \square p$.

$K$ is the weakest modal logic. In general, modal logics are extensions of the logic $K$ with additional schemas such as those listed in Figure 1. Let $\Delta$ denote a finite set of modal axiom schemas. By $K \Delta$ we denote the smallest modal logic which contains the schemas in $\Delta$. A modal formula $\varphi$ is a theorem of $K$ or its extension iff $\varphi$ can be derived from the axioms by using the rules of the logic.

The standard semantics of propositional modal logics, known as the Kripke semantics or possible world semantics, is given in terms of relational structures called frames. A frame of a modal logic is a pair $\mathcal{F}=(W, R)$ of a non-empty set of worlds $W$ and a binary relation $R$ on $W . R$ is the accessibility relation and determines the truth of modal formulae in possible worlds. The defining class of frames of a modal logic determines, and is determined by, a corresponding class of models. A (relational) model is a pair $\mathcal{M}=(\mathcal{F}, v)$ of a frame $\mathcal{F}$ and a valuation function $v$. $v$ assigns subsets of $W$ to atomic propositional variables. The model $\mathcal{M}$ is therefore said to be based on the frame $\mathcal{F}$. Truth in any model $\mathcal{M}=(W, R, v)$ and any world $x \in W$ is defined inductively by:

$$
\begin{array}{ll}
\mathcal{M}, x=p \text { iff } x \in v(p) & \mathcal{M}, x \models \psi \wedge \phi \text { iff both } \mathcal{M}, x \models \psi \text { and } \mathcal{M}, x \models \phi \\
\mathcal{M}, x \models \neg \psi \text { iff } \mathcal{M}, x \forall \forall & \mathcal{M}, x \models \square \psi \text { iff }(x, y) \in R \text { implies } \mathcal{M}, y \models \psi, \text { for any } y \in W
\end{array}
$$

A modal formula is valid in a frame iff it is valid in all models based on the frame. The basic modal logic $K$ is completely determined by the class of all frames $(W, R)$.

Normal modal logics can be studied systematically by considering the classes of frames they define. In general, these are subclasses of the class of all frames which define the basic modal logic $K$. A modal logic $K \Delta$ is said to be sound (respectively complete) with respect to a class of frames iff for any modal formula $\varphi$, any frame in the class validates $\varphi$ if (respectively iff) $\varphi$ is a theorem in $K \Delta$. A modal logic is said to be complete iff it is complete with respect to some class of frames. A class of frames comprising of all frames satisfying a set of first-order conditions is said to be an elementary class.

Except for logics based on McKinsey's axiom the logics considered in this paper are all Sahlqvist. That is, the logics are axiomatisable with Sahlqvist formulae as axiom schemas. The class of Sahlqvist formulae is a syntactically defined class of formulae satisfying the well-known Sahlqvist theorem [Sahlqvist 1975] which says that (i) all Sahlqvist formulae define first-order conditions on frames and these properties can be effectively computed (correspondence result), and that (ii) all Sahlqvist formulae are canonical, i.e. valid in their canonical frames and hence axiomatise completely the classes of frames satisfying the corresponding first-order properties (completeness result). Figure 1 lists the first-order correspondence properties satisfied by classes of frames for extensions $K \Delta$ for a selection of common schemas. However not every modal schema has an equivalent first-order frame property. For example, the logic $K M$ is not determined by any elementary class of frames. The formula $M$ is also not canonical.

We use the notational convention by which for example $K T 4$ denotes the smallest extension of $K$ in which both $T$ and 4 are schemas. Alternative names for $K T_{4}$ and $K T_{4} B$ are $S_{4}$ and $S 5$. Sometimes logics coincide, for example: $K T=K D T, K 4 B=K 5 B$, $S 5=K T 4 B=K D 4 B=K T 5$.

By definition, $\operatorname{Sf}(\varphi)$ denotes the set of all subformulae of $\varphi$ and $\operatorname{Sf}(X)=\{\psi \mid \psi \in$ $\operatorname{Sf}(\phi), \phi \in X\}$, where $X$ is a set of formulae.

\section{B. BASICS OF HYPERRESOLUTION AND PARAMODULATION}

For easy reference this section recalls the basics of hyperresolution and paramodulation for first-order clause logic. More detailed accounts can be found in [Bachmair and

ACM Transactions on Computational Logic, Vol. X, No. Y, August 2005. 
Ganzinger 2001] and references such as [Leitsch 1997; Robinson and Voronkov 2001; Socher-Ambrosius and Johann 1997].

\section{B.1 Notation}

First-order variables are denoted by $x, y, z$, terms are denoted by $s, t, u$, constants by $a, b$, functions by $f, g, h$, predicate symbols by $P, Q, R$, atoms by $A, B$, literals by $L$, clauses by $C$, formulae by $\varphi, \phi, \psi$, and sets of clauses by $N$. As usual the symbols $\vee, \wedge$ and $\neg$ denote logical disjunction, conjunction and negation, respectively.

\section{B.2 Clause logic}

A literal is an atomic formula $A$ (a positive literal) or the negation $\neg A$ of an atomic formula $A$ (a negative literal). If the predicate symbol of a literal has arity one (two) then we call this literal a unary literal (binary literal). By definition, in a unary clause (binary clause) all literals are unary (binary). We regard a clause as a multiset of literals. A multiset over a set $\mathcal{L}$ is a mapping $C$ from $\mathcal{L}$ to the natural numbers. We write $L \in C$ if $C(L)>0$ for a literal $L$. A subclause of a clause $C$ is a sub-multiset of $C$. We use $\emptyset$ to denote the empty clause. A positive (negative) clause contains only positive (negative) literals. The positive (negative) part of a clause $C$ is the subclause of all positive (negative) literals in $C$. A clause which consists of only one literal is called a unit clause. A split component of a clause $C \cup D$ is a subclause $C$ such that $C$ and $D$ do not have any variables in common, i.e. are variable disjoint. A maximally split (or variable indecomposable) clause cannot be partitioned (or split) into subclauses which do not share variables. Two clauses (or formulae) are said to be variants of each other iff they are equal modulo variable renaming. Variant clauses are assumed to be equal.

An expression is a term, an atom, a literal or a clause. An expression is called functional if it contains a constant or a function symbol, and non-functional, otherwise. The set of all free variables occurring in an expression $E$, or in a set of expressions $N$, is denoted as $\operatorname{var}(E)$ or $\operatorname{var}(N)$. An expression is called ground if it contains no variables. For sets of expressions, $|N|$ denotes the cardinality of the set $N$.

\section{B.3 Hyperresolution with splitting}

The hyperresolution calculus is denoted by $\mathrm{H}$. Inferences are computed with the following expansion rules.

Deduce: $\frac{N}{N \cup\{C\}}$

provided $C$ is the conclusion of applying one of the inference rules below to premises in $N$.

Delete: $\frac{N \cup\{C\}}{N}$

provided $C$ is a tautology or $N$ contains a clause which is a variant of $C$.

Splitting: $\frac{N \cup\left\{C_{1} \vee C_{2}\right\}}{N \cup\left\{C_{1}\right\} \mid N \cup\left\{C_{2}\right\}}$

provided $C_{1}$ and $C_{2}$ are variable disjoint.

The inference rules of $\mathrm{H}$ are the hyperresolution and positive factoring rule.

Hyperresolution: $\frac{C_{1} \vee A_{1} \quad \ldots \quad C_{n} \vee A_{n} \quad \neg B_{1} \vee \ldots \vee \neg B_{n} \vee D}{\left(C_{1} \vee \ldots \vee C_{n} \vee D\right) \sigma}$

where (i) $\sigma$ is the most general unifier such that $A_{i} \sigma=B_{i} \sigma$ for every $i, 1 \leq i \leq n$, and (ii) $C_{i} \vee A_{i}$ and $\mathrm{D}$ are positive clauses, for every $i, 1 \leq i \leq n$. The rightmost premise in the rule is referred to as the negative premise and all other premises are referred to as positive premises. 
Positive factoring: $\frac{C \vee A \vee B}{(C \vee A) \sigma}$

where $\sigma$ is the most general unifier of $A$ and $B$, and $C$ is a positive clause.

For problems with equality two additional inference rules are included as appropriate.

Paramodulation: $\frac{C D \vee s \approx t}{\left(C[t]_{\lambda} \vee D\right) \sigma}$

where $\left.C\right|_{\lambda}=s^{\prime}$ and $\sigma$ is the most general unifier of $s$ and $s^{\prime}$.

Reflexivity resolution: $\frac{s \not \approx s^{\prime} \vee C}{C \sigma}$

where $\sigma$ is the most general unifier of $s$ and $s^{\prime}$.

By definition the main premise of an inference step is the rightmost premise in the rule applied.

A derivation in $\mathrm{H}$ from a set of clauses $N$ is a finitely branching, ordered tree $T$ with root $N$ and nodes which are sets of clauses. The tree is constructed by applications of the expansion rules to the leaves. Any branch $N\left(=N_{0}\right), N_{1}, \ldots$ in a derivation $T$ is called a closed branch in $T$ iff the clause set $\bigcup_{j \geq 0} N_{j}$ contains the empty clause, otherwise it is called an open branch. We say a branch $\mathcal{B}$ in a derivation tree is complete (with respect to $\mathrm{H}$ ) iff no new successor nodes can be added to the branch of $\mathcal{B}$ within $\mathrm{H}$, otherwise it is called an incomplete branch. A derivation is complete iff all of its branches are either closed or complete. A derivation $T$ is a proof iff every branch $N\left(=N_{0}\right), N_{1}, \ldots$ in it is a closed branch, otherwise it is called an open derivation.

In general, the calculus $\mathrm{H}$ can be enhanced with standard simplification rules such as subsumption deletion, in fact, it can be enhanced by any simplification rule which is compatible with a general notion of redundancy [Bachmair and Ganzinger 2001; Bachmair et al. 1993]. A set $N$ of clauses is saturated up to redundancy with respect to a particular refinement of resolution if the conclusion of every inference from non-redundant premises in $N$ is either contained in $N$, or else is redundant in $N$.

A derivation $T$ from $N$ is called fair iff for any branch $N\left(=N_{0}\right), N_{1}, \ldots$ in $T$, with limit $N_{\infty}=\bigcup_{j \geq 0} \bigcap_{k \geq j} N_{k}$, it is the case that each clause $C$ which can be deduced from non-redundant premises in $N_{\infty}$ is contained in some $N_{j}$. Intuitively, fairness means that no non-redundant inferences are delayed indefinitely. For a finite complete branch $N\left(=N_{0}\right), N_{1}, \ldots N_{n}$, the limit $N_{\infty}$ is equal to $N_{n}$.

Theorem B.1 (Soundness and completeness of $\mathrm{H}$ ) Let $T$ be a fair $\mathrm{H}$-derivation from a set $N$ of clauses. Then: (i) If $N\left(=N_{0}\right), N_{1}, \ldots$ is a branch with limit $N_{\infty}$, then $N_{\infty}$ is saturated (up to redundancy). (ii) $N$ is satisfiable if and only if there exists a branch in $T$ with limit $N_{\infty}$ such that $N_{\infty}$ is satisfiable. (iii) $N$ is unsatisfiable if and only if for every branch $N\left(=N_{0}\right), N_{1}, \ldots$ the clause set $\bigcup_{j \geq 0} N_{j}$ contains the empty clause.

Proof. A consequence of the completeness of the superposition calculus with splitting [Bachmair et al. 1993].

In this paper we assume that a hyperresolution inference cannot use a clause $C$ as a positive premise if the splitting rule or the factoring rule can be applied to $C$. As usual we make the minimal assumption that no inference rule is applied twice to the same premises during the derivation. It is not difficult to see that any derivation in $\mathrm{H}$ generated according to these assumptions is fair and therefore sound and complete.

\section{PROOFS OF SOUNDNESS}

Theorem C.1 (Soundness of $\Pi_{\mathfrak{X}}^{\Delta}$ ) Let $L=K_{(m)} \Delta$ be an extension of multi-modal $K_{(m)}$, where $\Delta$ is a finite set of modal formulae. Let $\varphi$ be any $L$-formula and assume

ACM Transactions on Computational Logic, Vol. X, No. Y, August 2005. 
$\bigcup_{\mathcal{A} \in \Delta} \mathfrak{X}_{\mathcal{A}}$ is a finite set of $L$-formulae. If $\varphi$ is $L$-satisfiable then $\Pi_{\mathfrak{X}}^{\Delta}(\varphi)$ is first-order satisfiable, where $\mathfrak{X}=\left\{\mathfrak{X}_{\mathcal{A}}\right\}_{\mathcal{A} \in \Delta}$.

Proof. Let $\mathcal{M}=\left(W,\left\{\mathcal{R}_{i}\right\}_{i}, v\right)$ be an $L$-model for $\varphi$. Our aim is to construct a firstorder logic model $\mathcal{M}^{*}$ for $\Pi_{\mathfrak{X}}^{\Delta}(\varphi)$. W.l.o.g. we assume that instead of schema clauses, the corresponding schema formulae are used in the definition of $\Pi_{\mathfrak{X}}^{\Delta}(\varphi)$. By this we mean that $\operatorname{Ax}^{\mathcal{A}}(\psi)$ in part (2) of Definition 4.1 is not given by a conjunction of schema clause instances but by the corresponding instances of the formulae obtained by partial rewriting of the axiom $\mathcal{A}$ using the semantic definition of the connectives, leaving just the innermost modalities. For instance, the schema formula for axiom schema 4 is $\forall x\left(Q_{\square p}(x) \rightarrow \forall y\left(R(x, y) \rightarrow Q_{\square p}(y)\right)\right)$.

Now, construct $\mathcal{M}^{*}=\left(W,{ }^{I}\right)$ from $\mathcal{M}$ as follows. For every unary predicate symbol $Q_{\psi}$, (i) if $\psi$ is a propositional symbol $p$, let $Q_{p}^{I}=v(p)$, (ii) if $\psi=\neg \phi$, let $Q_{\neg \phi}^{I}=W \backslash Q_{\phi}^{I}$, (iii) if $\psi=\phi \wedge \phi^{\prime}$, let $Q_{\phi \wedge \phi^{\prime}}^{I}=Q_{\phi}^{I} \cap Q_{\phi^{\prime}}^{I}$, (iv) if $\psi=\phi \vee \phi^{\prime}$, let $Q_{\phi \vee \phi^{\prime}}^{I}=Q_{\phi}^{I} \cup Q_{\phi^{\prime}}^{I}$, (v) if $\psi=\square \phi$, let $Q_{\square \phi}^{I}=\left\{w \mid \forall v \in W\left(\mathcal{R}_{i}(w, v) \rightarrow \mathcal{M}, v \mid=\phi\right)\right\}$, (vi) if $\psi=\neg \square \phi$, let $Q_{\neg \square \phi}^{I}=\left\{w \mid \exists v \in W\left(\mathcal{R}_{i}(w, v) \wedge \mathcal{M}, v \not \models \phi\right)\right\}$. For every binary predicate symbol $R_{i}$ let $R_{i}^{I}=\mathcal{R}_{i}$.

Let $\psi$ be a subformula of $\varphi$. Using these definitions it is not difficult to prove (by induction on the structure of $\psi$ ) that, for any world $w \in W$,

$$
\begin{aligned}
& \mathcal{M}, w \models \psi \text { iff } \mathcal{M}^{*}, I[x / w] \models \pi(\psi, x), \text { and } \\
& \mathcal{M}, w \models \psi \text { iff } \mathcal{M}^{*}, I[x / w] \models Q_{\psi}(x) .
\end{aligned}
$$

Next, consider the definitions introduced by the translation. We want to show that for any definition $\operatorname{Def}(\psi)$ (for any $\psi \in \operatorname{Sf}^{-}(\varphi)$ ) and any world $w \in W, \mathcal{M}^{*}, I[x / w] \models$ $\operatorname{Def}(\psi)$. (a) If $\mathcal{M}, w \models \psi$ for $w \in W$ then $\mathcal{M}^{*}, I[x / w] \models \pi(\psi, x)$ and $\mathcal{M}^{*}, I[x / w] \models$ $Q_{\psi}(x)$, by (9) and (10). (b) For any world $w \in W$ such that $\mathcal{M}, w \not \neq \psi$ we have that $\mathcal{M}^{*}, I[x / w] \not \models \pi(\psi, x)$ and $\mathcal{M}^{*}, I[x / w] \not \models Q_{\psi}(x)$, also by (9) and (10). (a) and (b) imply that $\mathcal{M}^{*}, I[x / w] \models \forall x\left(Q_{\psi}(x) \leftrightarrow \pi(\psi, x)\right)$. Therefore, by the definition of $\operatorname{Def}(\psi)$ we can conclude that $\mathcal{M}^{*}, I[x / w] \mid=\operatorname{Def}(\psi)$ in all cases except when $\psi$ has the form $\neg \square \phi$. For the case that $\psi=\neg \square \phi$ it remains to show that $\mathcal{M}^{*}, I[x / w] \models \forall x\left(Q_{\neg \square \phi}(x) \leftrightarrow \neg Q_{\square \phi}(x)\right)$. This is clearly true by the definition of the interpretation of $Q_{\square \phi}$ and $Q_{\neg \square \phi}$ in (v) and (vi) above.

For definitions of subformulae in $X$ (part (3) of Definition 4.1) the proof is the same.

Finally consider $\operatorname{Ax}^{\mathcal{A}}(\psi)$ (in the form of schema formulae) for an arbitrary axiom schema $\mathcal{A} \in \Delta$ and an arbitrary $\psi \in \mathfrak{X}_{\mathcal{A}}$. $\operatorname{Ax}^{\mathcal{A}}(\psi)$ is obtained by partially rewriting the axiom $\mathcal{A}$ using the semantic definition of the connectives. This partial rewriting preserves satisfiability equivalence [Ohlbach 1998]. It follows therefore that $\mathcal{M}^{*}, I[\bar{x} / \bar{w}] \models \operatorname{Ax}^{\mathcal{A}}(\psi)$, where $\bar{x}$ are the variables occurring freely in $\operatorname{Ax}^{\mathcal{A}}(\psi)$ and $\bar{w}$ is a sequence of arbitrary worlds in $W$.

Since Skolemisation preserves satisfiability equivalence the result is true for the original definition of $\Pi_{\mathfrak{X}}^{\Delta}(\varphi)$ formulated in terms of schema clauses.

Theorem C.2 (Soundness of mixed reduction) Let $L$ be a propositional modal logic $K_{(m)} \Delta$ with $\Delta$ a finite set of modal formulae. Suppose $\Delta$ can be partitioned into two sets of axioms, $\Delta^{\prime}$ and $\Delta^{\prime \prime}$, so that all axioms in $\Delta^{\prime}$ are first-order definable. Assume $\bigcup_{\mathcal{A} \in \Delta^{\prime \prime}} \mathfrak{X}_{\mathcal{A}}$ is a finite set of $L$-formulae. Let $\mathfrak{X}=\left\{\mathfrak{X}_{\mathcal{A}}\right\}_{\mathcal{A} \in \Delta^{\prime \prime}}$ and let $\varphi$ be any $L$-formula. If $\varphi$ is $L$-satisfiable then $\operatorname{Corr}\left(\Delta^{\prime}\right) \wedge \Pi_{\mathfrak{X}}^{\Delta^{\prime \prime}}(\varphi)$ is first-order satisfiable.

Proof. The proof is similar to the proof of the previous theorem. 


\section{PROOFS OF COMPLETENESS FOR FAMILIAR AXIOMS}

For convenience we introduce the following terminology. A positive ground clause consisting only of unary literals is called a state clause, and a positive ground binary unit clause is called a transition clause.

Theorem D.1 Let $\varphi$ be any modal formula and assume $\mathfrak{X}=\left\{\mathfrak{X}_{\varphi}^{\epsilon}\right\}$. Then, $\Pi_{\mathfrak{X}}^{B}(\varphi)$ is unsatisfiable in first-order logic, whenever $\varphi$ is unsatisfiable in $K B$.

Proof. We prove that any $\mathrm{H}$-refutation of (the clausal form of $) \operatorname{Corr}(\{B\}) \wedge \Pi(\varphi)$ can be mapped to an $\mathrm{H}$-refutation of (the clausal form of) $\Pi_{\mathfrak{X}}^{B}(\varphi)$. The result follows then by the completeness of the standard translation and the completeness of $K B$.

Let $M=\operatorname{Cls}(\operatorname{Corr}(\{B\}) \wedge \Pi(\varphi))$ and $N=\operatorname{Cls}\left(\Pi_{\mathfrak{X}}^{B}(\varphi)\right)$. Our aim is to prove that any $\mathrm{H}$-derivation from $M$ can be mapped to a derivation from $N$. This is done by a simulation of each inference step using the classical approach.

The clausal sets $M$ and $N$ are identical, except that whereas $M$ contains the clause $\mathrm{Cls}(\operatorname{Corr}(\{B\}))$, i.e. the symmetry clause

$$
\neg R(x, y) \vee R(y, x),
$$

$N$ contains instances of the clausal schema for $B$, i.e.

$$
\neg R(x, y) \vee \neg Q_{\square \psi}(y) \vee Q_{\psi}(x), \quad \text { where } \square \psi \in \operatorname{Sf}(\varphi),
$$

and positive shortcut clauses of the form $Q_{\sim \psi}(x) \vee Q_{\psi}(x)$ (from (4) of Definition 4.1).

In hyperresolution derivations the empty clause is the conclusion of an inference step with a negative clause and positive clauses. This means that the empty clause cannot be derived directly with the symmetry clause (11). Negative clauses have the form $\neg Q_{\neg \psi}(x) \vee$ $\neg Q_{\psi}(x)$ or $\neg Q_{\perp}(x)$. Any negative clause in $M$ is also in $N$, and vice versa. Important for the derivation from $M$ of the empty clause are therefore positive premises with unary predicate symbols. These are state clauses, since all positive premises in $\mathrm{H}$-derivations from $M$ are ground. As a consequence, it suffices to prove the following:

$(P B)$ If $M_{0}, M_{1}, \ldots, M_{n}$ is a branch in the $\mathrm{H}$-derivation from $M_{0}=M$, then it is possible to construct a branch $N_{0}, N_{1}, \ldots, N_{k}$ in an $\mathrm{H}$-derivation starting from $N_{0}=N$, for some $k$ with $n \geq k \geq 0$, and the following are true.

(1) Every state clause in $M_{n}$ occurs also in $N_{k}$.

(2) For every transition clause $R(s, t)$ in $M_{n}$ but not in $N_{k}, N_{k}$ contains the transition clause $R(t, s)$.

The proof of $(P B)$ is by induction on the length $n$ of the derivation starting with $M$, i.e. using the frame correspondence property.

Base case. If $n=0$ then the derivation consists of the single node $M_{0}=M$. Let $k=0$ and $N_{0}=N$. Clearly $(P B)$ is true since $M$ and $N$ differ only with respect to the theory clauses and the shortcut clauses they contain.

Inductive step. Suppose that $(P B)$ holds for any derivation from $M$ of length $n$. We show that the claim holds also for derivations of length $n+1$. The proof is by case analysis of inferences with a main (i.e. non-positive) premise $C$ from $M_{n}$ and conclusion $D$ in $M_{n+1}$. We show the existence of a $k^{\prime} \geq k$ and a set $N_{k^{\prime}}$ derived from $N_{k}$ by at most one inference step such that $(P B)$ holds for $M_{n+1}$ and $N_{k^{\prime}}$.

Observe $C \in N_{k}$, except when $C$ is the symmetry clause (11). This is not difficult to see. Derivations from $M$ have the property that a main premise $C$ has three forms: (i) the clause (11), (ii) a definitional clause, or (iii) $C$ is the premise of a splitting or factoring step. In case (ii), clearly $C \in N_{k}$. In case (iii), $C$ is a ground, positive clause and contains only unary predicate symbols, and hence $C \in N_{k}$, by the induction hypothesis.

ACM Transactions on Computational Logic, Vol. X, No. Y, August 2005. 
Now, we consider the possible forms that inference steps with $C \in M_{n}$ as main premise and $D \in M_{n+1}$ as conclusion might have and construct a corresponding sequence of inferences producing $N_{k^{\prime}}$ from $N_{k}$ so that property $(P B)$ is true.

Case 1: $C$ is a definitional clause associated with a Boolean subformula or a $\neg \square$ subformula. In all cases the positive premises are state clauses and belong to both $M_{n}$ and $N_{k}$ by the induction hypothesis. $D$ is a state clause. Thus, we let $k^{\prime}=k+1$ and $N_{k^{\prime}}=N_{k} \cup\{D\}$, which is obtained by the same inference as $M_{n+1}$ from $M_{n}$. Evidently, the property $(P B)$ is true for $M_{n+1}$ and $N_{k^{\prime}}$.

Case 2: $C$ is a positive ground clause $D \vee D^{\prime}$ and $D$ is the conclusion of applying splitting or factoring to $C$. $C$ and $D$ are state clauses. Hence we let $k^{\prime}=k+1$ and $N_{k^{\prime}}=N_{k} \cup\{D\}$, which is obtained by the same inference as $M_{n+1}$ from $M_{n}$. Therefore, $(P B)$ is true for $M_{n+1}$ and $N_{k^{\prime}}$.

Case 3: $C$ is the symmetry clause (11) and $D$ is derived with one positive premise $R(s, t)$ in $M_{n}$. Then $D=R(t, s)$. (a) Suppose $R(s, t) \in N_{k}$. Since $D \in M_{n+1}$ is the converse of a clause in $N_{k}$ property $(P B)$ holds for $M_{n+1}$ and $N_{k^{\prime}}$. (b) Suppose $R(s, t) \notin N_{k}$. Then by the induction hypothesis $D=R(t, s) \in N_{k}$ and the property $(P B)$ holds for $M_{n+1}$ and $N_{k^{\prime}}$. In all cases we let $k^{\prime}=k$, i.e. $N_{k^{\prime}}=N_{k}$.

Case 4: $C$ is a definitional clause associated with a $\square$ subformula, i.e. $C=\neg Q_{\square \psi}(x) \vee$ $\neg R(x, y) \vee Q_{\psi}(y)$. Assume $D=Q_{\psi}(t)$ is derived with $C$ and two premises $Q_{\square \psi}(s)$ and $R(s, t)$ in $M_{n}$. (Since factoring and splitting is applied as early as is possible we can assume that the state premise is a unit clause and $D$ is a state clause.) By the induction hypothesis, $Q_{\square \psi}(s)$ is also in $N_{k}$. (a) If $R(s, t) \in N_{k}$ then we let $k^{\prime}=k+1$ and $N_{k^{\prime}}=N_{k} \cup\{D\}$, which is obtained by the same inference as $M_{n+1}$ from $M_{n}$. (b) If $R(s, t) \notin N_{k}$ then, by the induction hypothesis, $N_{k}$ contains the clause $R(t, s)$. Hence, $D$ can be derived from $N_{k}$ using $Q_{\square \psi}(s), R(t, s)$ and the schema clause (12). Therefore, we can let $N_{k^{\prime}}=N_{k} \cup\{D\}$ and $k^{\prime}=k+1$. In both cases it follows that $(P B)$ is true for $M_{n+1}$ and $N_{k^{\prime}}$.

Theorem D.2 Suppose $\mathcal{A} \in\{T, 4\}$. Let $\varphi$ be any modal formula and assume $\mathfrak{X}=\left\{\mathfrak{X}_{\varphi}^{\epsilon}\right\}$. Then, $\Pi_{\mathfrak{X}}^{\mathcal{A}}(\varphi)$ is unsatisfiable in first-order logic, whenever $\varphi$ is unsatisfiable in $K \mathcal{A}$.

Proof. The idea of the proof for $\mathcal{A}=T$ is exactly the same as in the proof of the previous theorem. The only difference is that the simulating derivation which we need to construct satisfies the property:

$(P T)$ If $M_{0}, M_{1}, \ldots, M_{n}$ is a branch in the $\mathrm{H}$-derivation from $M_{0}=M$, then it is possible to construct a branch $N_{0}, N_{1}, \ldots, N_{k}$ in an $\mathrm{H}$-derivation starting from $N_{0}=N$, where $k=n$ and the following is true.

(1) Every state clause in $M_{n}$ occurs also in $N_{k}$.

(2) Every transition clause of the form $R(s, f(s))$ in $M_{n}$ occurs also in $N_{k}$.

Such a derivation is not difficult to construct. The details are therefore omitted.

More interesting is the case for K4. Again the idea of the proof is the same. The property to be proved is:

$\left(P_{4}\right)$ If $M_{0}, M_{1}, \ldots, M_{n}$ is a branch in the $\mathrm{H}$-derivation from $M_{0}=M$, then it is possible to construct a branch $N_{0}, N_{1}, \ldots, N_{k}$ in an $\mathrm{H}$-derivation starting from $N_{0}=N$, for some $k$ with $k \geq n$, and the following are true.

(1) Every state clause in $M_{n}$ occurs also in $N_{k}$.

(2) For every transition clause $R(s, t)$ in $M_{n}$ but not in $N_{k}$ and $s \neq t$,

(a) $N_{k}$ contains a chain of transition clauses

$$
R\left(u_{1}, u_{2}\right), R\left(u_{2}, u_{3}\right), \ldots, R\left(u_{m-1}, u_{m}\right), R\left(u_{m}, u_{m+1}\right)
$$


such that $u_{1}=s$ and $u_{m+1}=t$, and

(b) if $Q_{\square \psi}(s) \in N_{k}$, then $Q_{\square \psi}\left(u_{i}\right) \in N_{k}$ for every $i, 1 \leq i \leq m$.

Properties (a) and (b) are crucial, for they imply that for any transition clause $R(s, t) \in$ $M_{n}$ that was derived with the transitivity clause

$$
\neg R^{2}(x, y) \vee R(x, y),
$$

and is therefore not in $N_{k}$, any inference that produces state clauses can be simulated. The only inference that produces such a state clause is with a $\square$ definitional clause. That is, when $\left\{Q_{\square \psi}(s), R(s, t)\right\} \subseteq M_{n}$ then an inference with $\neg Q_{\square \psi}(x) \vee \neg R(x, y) \vee Q_{\psi}(y)$ produces $Q_{\psi}(t)$. What is important about (b) is that $Q_{\square \psi}\left(u_{m}\right) \in N_{k}$, when $Q_{\square \psi}(s) \in N_{k}$. As a consequence $Q_{\psi}(t)$ can be derived with $Q_{\square \psi}\left(u_{m}\right), R\left(u_{m}, u_{m+1}\right)$ and $\neg Q_{\square \psi}(x) \vee$ $\neg R(x, y) \vee Q_{\psi}(y)$.

The proof of $\left(P_{4}\right)$ is by induction on the length $n$ of the derivation starting with $M$. The base case, and Cases 1 and 2 of the inductive step (i.e. the simulation of inferences with a Boolean or a $\neg \square$ definitional clause, and factoring, and splitting) are as in the proof of Theorem D.1.

Case 3: $C$ is the transitivity clause (13) and $D$ is derived with two positive premises in $M_{n}$. Suppose the positive premises are transition clauses of the form $R(s, v), R(v, t)$. Then $D=R(s, t)$. Since $R(s, t)$ cannot be derived from $N_{k}$, we need to show the existence of inferences that ensure that the properties of $\left(P_{4}\right)$ are true. Property $(P 4.1)$ holds trivially. For property $(P 4.2)$ the derivation from $N_{k}$ needs to be continued in such a way that, for any subformula $\square \psi$ such that $Q_{\square \psi}(s)$ is in $N_{k}$, there is a clause $Q_{\square \psi}\left(v^{\prime}\right)$ in $N_{k}$ such that $v^{\prime}$ is a predecessor of $t$ in $N_{k}$. (Then $Q_{\psi}(t)$ can be derived in a subsequent step.)

Suppose $Q_{\square \psi}(s) \in N_{k}$ and $\{R(s, v), R(v, t)\} \subseteq N_{k}$. Then, unless $Q_{\square \psi}(v)$ does not already belong to $N_{k}$, it can be derived by one hyperresolution inference step using $Q_{\square \psi}(s)$, the clauses $R(s, v)$, and the clausal schema instance $\neg Q_{\square \psi}(x) \vee \neg R(x, y) \vee Q_{\square \psi}(y)$.

However it may be that either $R(s, v)$, or $R(v, t)$, or both are not present in $N_{k}$. For both clauses, the induction hypothesis applies. This implies for $C^{\prime}=R(s, v)\left(\right.$ or $\left.C^{\prime}=R(v, t)\right)$ and $C^{\prime} \notin N_{k}$ there is a chain of transition clauses

$$
R\left(w_{1}, w_{2}\right), R\left(w_{2}, w_{3}\right), \ldots, R\left(w_{m^{\prime}-1}, w_{m^{\prime}}\right), R\left(w_{m^{\prime}}, w_{m^{\prime}+1}\right)
$$

in $N_{k}$, for $m^{\prime} \geq 2$, such that $C^{\prime}=R\left(w_{1}, w_{m^{\prime}+1}\right)$. Thus we can conclude $N_{k}$ contains a chain of transition clauses

$$
R\left(u_{1}, u_{2}\right), R\left(u_{2}, u_{3}\right), \ldots, R\left(u_{m-1}, u_{m}\right), R\left(u_{m}, u_{m+1}\right)
$$

with $m \geq 2$, such that $u_{1}=s$ and $u_{m+1}=t$. Now, since $Q_{\square \psi}(s) \in N_{k}$ and using (14) and the schema clause

$$
\neg Q_{\square \psi}(x) \vee \neg R(x, y) \vee Q_{\square \psi}(y), \quad \text { where } \square \psi \in \operatorname{Sf}(\varphi),
$$

we can iteratively derive $Q_{\square \psi}\left(u_{l}\right)$, for each $l$ such that $1 \leq l \leq m$. The last clause derived is $Q_{\square \psi}\left(u_{m}\right) . u_{m}$ is therefore a predecessor of $t$ satisfying the required property.

We have thus shown that a bounded number of $\mathrm{H}$-inferences exist which produce a set $N_{k^{\prime}}$ satisfying $\left(P_{4}\right)$ for an arbitrary subformula $\square \psi$.

Case 4: $C$ is a definitional clause associated with a $\square$ subformula, i.e. $C=\neg Q_{\square \psi}(x) \vee$ $\neg R(x, y) \vee Q_{\psi}(y)$. Assume $D$ is derived with $C$ and two premises $Q_{\square \psi}(s)$ and $R(s, t)$ in $M_{n}$. By the induction hypothesis, $Q_{\square \psi}(s)$ is also in $N_{k}$. (a) If $R(s, t) \in N_{k}$ then we let $k^{\prime}=k+1$ and $N_{k^{\prime}}=N_{k} \cup\{D\}$, which is obtained by the same inference as $M_{n+1}$ from $M_{n}$. (b) If $R(s, t) \notin N_{k}$ then, by the induction hypothesis, $N_{k}$ contains a chain $R\left(u_{1}, u_{2}\right), \ldots, R\left(u_{m-1}, u_{m}\right), R\left(u_{m}, u_{m+1}\right)$, such that $u_{1}=s$ and $u_{m+1}=t$, and $Q_{\square \psi}\left(u_{i}\right) \in$ $N_{k}$ for all $i, 1 \leq i \leq m$. In particular, $Q_{\square \psi}\left(u_{m}\right) \in N_{k}$ and $R\left(u_{m}, t\right)\left(=R\left(u_{m}, u_{m+1}\right)\right) \in N_{k}$. 
Hence, $D$ can be derived from $N_{k}$ using $C$. Therefore, we can let $N_{k^{\prime}}=N_{k} \cup\{D\}$ and $k^{\prime}=k+1$.

Theorem D.3 Let $\varphi$ be any modal formula and assume $\mathfrak{X}=\left\{\mathfrak{X}_{\varphi}^{\epsilon}\right\}$. Then, $\Pi_{\mathfrak{X}}^{D}(\varphi)$ is unsatisfiable in first-order logic, whenever $\varphi$ is unsatisfiable in $K D$.

Proof. In this proof we prove the following relationship between the derivations from $M=\operatorname{Cls}(\operatorname{Corr}(\{D\}) \wedge \Pi(\varphi))$ and $N=\operatorname{Cls}\left(\Pi_{\mathfrak{X}}^{D}(\varphi)\right)$ : There is a function $h$ from the Herbrand universe of $M$ to the Herbrand universe of $N$ such that $h$, homomorphicly extended to clauses, satsifies that for any state clause $C$ in the derivation from $M$, the state clause $h(C)$ occurs in the derivation from $N$. As a consequence, since the derivation of the empty clause involves at least one state clause and no theory clause, whenever the empty clause can be derived at $s$ (involving a premise $Q(s)$ ) then the empty clause can be derived from $N$ at $h(s)$ (involving the premise $Q(h(s))$ ). We define the function $h$ by induction on the length of the derivation from $M$ such that the following property is true.

$(P D)$ If $M_{0}, M_{1}, \ldots, M_{n}$ is a branch in the $\mathrm{H}$-derivation from $M_{0}=M$, then it is possible to construct a branch $N_{0}, N_{1}, \ldots, N_{k}$ in an $\mathrm{H}$-derivation starting from $N_{0}=N$, for some $k$ with $2 n \geq k \geq n$, and the following are true.

(1) For each state clause $C$ in $M_{n}, N_{k}$ contains the state clause $h(C)$.

(2) For each transition clause $R(s, t)$ in $M_{n}, N_{k}$ contains the transition clause $R(h(s), h(t))$.

The clausal sets $M$ and $N$ are identical, except that whereas $M$ contains the seriality clause, i.e.

$$
R(x, f(x)),
$$

$N$ contains instances of the $D$ schema clauses, i.e.

$$
\neg Q_{\square p}(x) \vee Q_{\neg \square \neg p}(x) \quad \text { where } \square \psi \in \operatorname{Sf}(\varphi),
$$

and positive shortcut clauses from (4) of Definition 4.1.

Base case. If $n=0$ then the derivation consists of the single node $M_{0}=M$. Let $k=0$ and $N_{0}=N$. $M$ and $N$ both contain one state clause, but no transition clause. W.l.o.g. suppose the state clause is $Q_{\varphi}(a)$ in both cases, where $\varphi$ is the candidate formula. Let $h(a)=a$. Clearly $(P D)$ is true for $M$ and $N$.

Inductive step. Suppose that the property $(P D)$ is true for any derivation from $M$ of length $n$. We show that the claim holds also for derivations of length $n+1$. The proof is by case analysis of inferences with a main (i.e. non-positive) premise $C$ from $M_{n}$ and conclusion $D$ in $M_{n+1}$. We show the existence of a $k^{\prime} \geq k$, a set $N_{k^{\prime}}$ derived from $N_{k}$ by zero or more inference steps, and an extension of the mapping $h$ such that $(P D)$ is true.

Case 1: $C$ is a definitional clause associated with a Boolean subformula or a $\neg \square$ subformula. By the induction hypothesis, in all cases the positive premises are state clauses $C^{\prime}$ in $M_{n}$ which have counterparts, $h\left(C^{\prime}\right)$, in $N_{k}$. Thus, we let $k^{\prime}=k+1$ and $N_{k^{\prime}}=N_{k} \cup\{h(D)\}$, which is obtained by the same inference as $M_{n+1}$ from $M_{n}$ and extend the definition of $h$ so that the correspondence between state clauses, and between transition clauses, is preserved.

Case 2: $C$ is a positive ground clause $D \vee D^{\prime}$ and $D$ is the conclusion of applying splitting or factoring to $C$. Proceed as in Case 1.

Case 3: Since $C$ is assumed to be negative but the seriality clause is positive, this case is vacuous. Inferences with the seriality clause are considered in subcase 4.(b) below.

Case 4: $C$ is a definitional clause associated with a $\square$ subformula, i.e. $C=\neg Q_{\square \psi}(x) \vee$ $\neg R(x, y) \vee Q_{\psi}(y)$. Assume $D$ is derived with $C$, a state clause $Q_{\square \psi}(s)$ and a clause 
$R(s, t)$ in $M_{n}$. By the induction hypothesis, $Q_{\square \psi}(h(s)) \in N_{k}$. (a) If $R(s, t)$ is a transition clause, then $R(h(s), h(t)) \in N_{k}$ by the induction hypothesis. Hence let $k^{\prime}=k+1$ and $N_{k^{\prime}}=N_{k} \cup\left\{Q_{\psi}(h(s))\right\}$, which is obtained by the same inference as $M_{n+1}$ from $M_{n}$.

(b) If $R(s, t)$ is not a transition clause, then it is the seriality clause and $D=Q_{\psi}(f(s))$. The simulating inference is the following. First, use $Q_{\square \psi}(h(s))$ and the schema clause (17) to derive $Q_{\neg \square \neg \psi}(h(s))$. Then derive $Q_{\psi}(g(h(s)))$ in one hyperresolution inference step with one of the definitional clauses associated with $\neg \square \neg \psi$, namely $\neg Q_{\neg \square \neg \psi}(x) \vee Q_{\psi}(g(x))$. Hence let $k^{\prime}=k+2$ and $N_{k^{\prime}}=N_{k} \cup\left\{Q_{\neg \square \neg \psi}(h(s)), Q_{\psi}(g(h(s)))\right\}$. Now let $h(f(s))=$ $g(h(s))$. It is not difficult to see that property $(P D)$ is satisfied.

Inspection of the proofs in this section reveals that the positive shortcut clauses are not needed for the simulations and may therefore be omitted from the definition of the axiomatic translation of $K B, K T, K 4$ and $K D$.

\section{E. PROOFS OF COMPLETENESS FOR GENERALISED AXIOMS}

\section{E.1 Completeness for $K \oplus \square p \rightarrow \square^{+} p$}

In this section the formula $\square p \rightarrow \square^{\kappa} \square p$ is denoted by $4^{\kappa}$ and we assume $\kappa \geq 1$.

Theorem E.1 $K 4^{\kappa}$ is complete with respect to the class of frames satisfying (the universal closure of)

$$
\neg R^{\kappa+1}(x, y) \vee R(x, y) .
$$

Proof. By Sahlqvist's [1975] Theorem.

Theorem E.2 (Completeness of $\Pi_{\mathfrak{X}}^{4^{\kappa}}$ ) Let $4^{\kappa}$ be the formula $\square p \rightarrow \square^{\kappa} \square p$ for a nonzero positive integer $\kappa$. Let $\varphi$ be any modal formula and assume $\mathfrak{X}=\left\{\mathfrak{X}_{4^{\kappa}}\right\}$ where $\square \mathfrak{X}_{4^{\kappa}}$ is the set of $\square$ subformulae of $\varphi$. If $\varphi$ is unsatisfiable in $K 4^{\kappa}$ then $\Pi_{\mathfrak{X}}^{4^{\kappa}}(\varphi)$ is unsatisfiable in first-order logic.

Proof. Let $M=\operatorname{Cls}\left(\operatorname{Corr}\left(\left\{4^{\kappa}\right\}\right) \wedge \Pi(\varphi)\right)$ and $N=\operatorname{Cls}\left(\Pi_{\mathfrak{X}}^{4^{\kappa}}(\varphi)\right)$. Our aim is to prove that any $\mathrm{H}$-derivation from $M$ can be mapped to a derivation from $N . M$ and $N$ are identical, except that whereas $M$ contains $\operatorname{Cls}\left(\operatorname{Corr}\left(\left\{4^{\kappa}\right\}\right)\right)$, i.e. the clause (18), $N$ contains instances of the clausal schema for $4^{\kappa}$, i.e.

$$
\neg Q_{\square \psi}(x) \vee \neg R^{\kappa}(x, y) \vee Q_{\square \psi}(y), \quad \text { where } \square \psi \in \operatorname{Sf}(\varphi),
$$

and positive shortcut clauses from (4) of Definition 4.1 . The property we want to prove is the following:

$\left(P_{4}{ }^{\kappa}\right)$ If $M_{0}, M_{1}, \ldots, M_{n}$ is a branch in the $\mathrm{H}$-derivation from $M_{0}=M$, then it is possible to construct a branch $N_{0}, N_{1}, \ldots, N_{k}$ in an H-derivation starting from $N_{0}=N$, for some $k$ with $k \geq n$, and the following are true.

(1) Every state clause in $M_{n}$ occurs also in $N_{k}$.

(2) For every transition clause $R(s, t)$ in $M_{n}$ but not in $N_{k}$ and $s \neq t$,

(a) $N_{k}$ contains a chain of transition clauses

$$
R^{\kappa}\left(u_{1}, u_{2}\right), R^{\kappa}\left(u_{2}, u_{3}\right), \ldots, R^{\kappa}\left(u_{m-1}, u_{m}\right), R\left(u_{m}, u_{m+1}\right)
$$

such that $u_{1}=s$ and $u_{m+1}=t$, and

(b) if $Q_{\square \psi}(s) \in N_{k}$, then $Q_{\square \psi}\left(u_{i}\right) \in N_{k}$ for all $i, 1 \leq i \leq m$.

Property $\left(P_{4}{ }^{\kappa}\right)$ does not make sense if $s=t$, hence the proviso that $s \neq t$. Only if the given set $M$ (and therefore also $N$ ) contain clauses of the form $R(s, s)$ can it be that $M_{n}$

ACM Transactions on Computational Logic, Vol. X, No. Y, August 2005. 
contains a clause $R(s, t)$ such that $s=t$. Even then, this clause must have been present from the outset. This implies that the $u_{i}$ in $\left(P 4^{\kappa} .2\right.$.a) can be taken to be pairwise distinct.

Properties (a) and (b) are important, for they imply that for any transition clause $R(s, t) \in M_{n}$ that was derived with the theory clause (18) (and is therefore not in $N_{k}$ ), any inference that produces state clauses can be simulated. The only inference with a transition clause which produces a state clause is with a $\square$ definitional clause. That is, when $\left\{Q_{\square \psi}(s), R(s, t)\right\} \subseteq M_{n}$ then an inference with $\neg Q_{\square \psi}(x) \vee \neg R(x, y) \vee Q_{\psi}(y)$ produces $Q_{\psi}(t)$. What is important about (b) is that $Q_{\square \psi}\left(u_{m}\right) \in N_{k}$, when $Q_{\square \psi}(s) \in N_{k}$. As a consequence $Q_{\psi}(t)$ can be derived with $Q_{\square \psi}\left(u_{m}\right), R\left(u_{m}, u_{m+1}\right)$ and $\neg Q_{\square \psi}(x) \vee$ $\neg R(x, y) \vee Q_{\psi}(y)$.

Of interest in the proof of $\left(P_{4}{ }^{\kappa}\right)$ are the cases where $C$ is the theory clause and $C$ is the definitional clause for $\square$. The other cases are the same as before and are therefore omitted.

Case 3: $C$ is the clause (18) and $D$ is derived with $\kappa+1$ positive premises in $M_{n}$. Suppose the positive premises are transition clauses of the form $R^{\kappa}(s, v), R(v, t)$. Then $D=R(s, t)$. Since $R(s, t)$ cannot be derived from $N_{k}$, we need to show the existence of inferences that ensure that the properties of $\left(P 4^{\kappa}\right)$ are true. Property $\left(P 4^{\kappa} .1\right)$ holds evidently. For property $\left(P 4^{\kappa} .2\right)$ the derivation from $N_{k}$ needs to be continued in such a way that, for any subformula $\square \psi$ such that $Q_{\square \psi}(s)$ is in $N_{k}$, there is a clause $Q_{\square \psi}\left(v^{\prime}\right)$ in $N_{k}$ such that $v^{\prime}$ is a predecessor of $t$ in $N_{k}$. (Then $Q_{\psi}(t)$ could be derived in a subsequent step.)

Suppose $Q_{\square \psi}(s) \in N_{k}$ and $U=\left\{R^{\kappa}(s, v), R(v, t)\right\} \subseteq N_{k}$. Then, unless $Q_{\square \psi}(v)$ does not already belong to $N_{k}$, it can be derived by one hyperresolution inference step using $Q_{\square \psi}(s)$, the clauses $R^{\kappa}(s, v)$, and the clausal schema instance $\neg Q_{\square \psi}(x) \vee \neg R^{\kappa}(x, y) \vee Q_{\square \psi}(y)$.

However it may be that some clauses in $U$ are not present in $N_{k}$. For any clause $C^{\prime} \in U$ such that $C^{\prime} \notin N_{k}$, the induction hypothesis applies. In particular, there is a chain of transition clauses

$$
R^{\kappa}\left(w_{1}, w_{2}\right), R^{\kappa}\left(w_{2}, w_{3}\right), \ldots, R^{\kappa}\left(w_{m^{\prime}-1}, w_{m^{\prime}}\right), R\left(w_{m^{\prime}}, w_{m^{\prime}+1}\right)
$$

in $N_{k}$, for $m^{\prime} \geq 2$, such that $C^{\prime}=R\left(w_{1}, w_{m^{\prime}+1}\right)$. The number of clauses in every such chain is $d^{\prime} \kappa+1$ for some integer $d^{\prime} \geq 1$. Therefore if we take the chain of clauses in $U$ and replace each clause in $U$, but not in $N_{k}$, by such a chain of length $d_{i} \kappa+1$, which we can do by the induction hypothesis, then we can conclude:

Lemma E.3 $N_{k}$ contains a chain of transition clauses

$$
R^{\kappa}\left(u_{1}, u_{2}\right), R^{\kappa}\left(u_{2}, u_{3}\right), \ldots, R^{\kappa}\left(u_{m-1}, u_{m}\right), R\left(u_{m}, u_{m+1}\right)
$$

with $m \geq 2$, such that $u_{1}=s$ and $u_{m+1}=t$. The length of the chain is $d \kappa+1$ for some integer $d \geq 1$.

ProOF. Rather than giving a formal proof we give an illustration of the essence of the argument. Suppose the following depicts the chain $U=\left\{R^{\kappa}(s, v), R(v, t)\right\}$, with length $\kappa+1$, where dashed links represent transition clauses not in $N_{k}$. Suppose there are $j$ transition clauses in $U$ but not in $N_{k}$, i.e. there are $j$ dashed links in the following picture.

Replacing the dashed links gives:

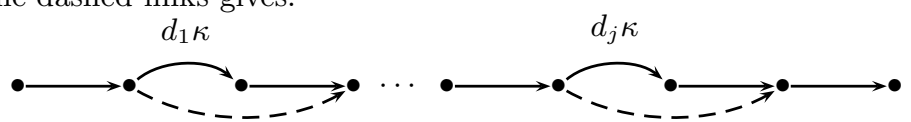

Each dashed link is replaced by a chain of transition clauses in $N_{k}$ (which exist by the induction hypothesis). Observe that the lengths of each of these chains is $d_{i} \kappa+1$. Now 
note that the length of the entire chain is $(\kappa+1)+d_{1} \kappa+\ldots+d_{j} \kappa$, i.e. $d \kappa+1$ for some integer $d \geq 1$.

Now, since $Q_{\square \psi}(s) \in N_{k}$, using (*) and the clause schema (19) we can iteratively derive $Q_{\square \psi}\left(u_{l}\right)$, for each $l$ such that $1 \leq l \leq m$. The last clause derived is $Q_{\square \psi}\left(u_{m}\right)$. $u_{m}$ is therefore a predecessor of $t$ satisfying the required property.

We have thus shown that a bounded number of $\mathrm{H}$-inferences exists which produce a set $N_{k^{\prime}}$ satisfying $\left(P_{4}{ }^{\kappa}\right)$ for an arbitrary subformula $\square \psi$.

Case 4: $C$ is a definitional clause associated with a $\square$ subformula, i.e. $C=\neg Q_{\square \psi}(x) \vee$ $\neg R(x, y) \vee Q_{\psi}(y)$. Assume $D$ is derived with $C$ and two premises $Q_{\square \psi}(s)$ and $R(s, t)$ in $M_{n}$. By the induction hypothesis, $Q_{\square \psi}(s)$ is also in $N_{k}$. If $R(s, t) \in N_{k}$ then we let $k^{\prime}=k+1$ and $N_{k^{\prime}}=N_{k} \cup\{D\}$, which is obtained by the same inference as $M_{n+1}$ from $M_{n}$.

If $R(s, t) \notin N_{k}$ then, by the induction hypothesis, $N_{k}$ contains a chain

$$
R^{\kappa}\left(u_{1}, u_{2}\right), R^{\kappa}\left(u_{2}, u_{3}\right), \ldots, R^{\kappa}\left(u_{m-1}, u_{m}\right), R\left(u_{m}, u_{m+1}\right),
$$

such that $u_{1}=s$ and $u_{m+1}=t$, and $Q_{\square \psi}\left(u_{i}\right) \in N_{k}$ for all $i, 1 \leq i \leq m$. In particular, $Q_{\square \psi}\left(u_{m}\right) \in N_{k}$ and $R\left(u_{m}, t\right)\left(=R\left(u_{m}, u_{m+1}\right)\right) \in N_{k}$. Hence, $D$ can be derived from $N_{k}$ using $C$. Therefore, we can let $N_{k^{\prime}}=N_{k} \cup\{D\}$ and $k^{\prime}=k+1$.

Inspection of the proof reveals that the positive shortcut clauses $Q_{\sim \psi}(x) \vee Q_{\psi}(x)$ are inessential for the completeness of the axiomatic encoding and may be omitted.

\section{E.2 Completeness for $K \oplus \diamond^{+} p \rightarrow \square^{+} p$}

Let $a l t_{1}^{\kappa_{1}, \kappa_{2}}$ be the formula $\diamond^{\kappa_{1}} \diamond p \rightarrow \square^{\kappa_{2}} \square p$, or equivalently $\neg \square^{\kappa_{1}} \neg \square p \rightarrow \square^{\kappa_{2}} \square \neg p$, for some positive integers $\kappa_{1}, \kappa_{2} \geq 0$.

Theorem E.4 Kalt ${ }_{1}^{\kappa_{1}, \kappa_{2}}$ is complete with respect to the class of frames satisfying the clause

$$
\neg R^{\kappa_{1}+1}(x, y) \vee \neg R^{\kappa_{2}+1}(x, z) \vee y \approx z .
$$

Proof. By Sahlqvist's [1975] Theorem.

Theorem E.5 (Completeness of $\Pi_{\mathfrak{X}}^{a l t_{1}^{\kappa_{1}, \kappa_{2}}}$ ) Let $\varphi$ be any modal formula and assume $\mathfrak{X}=\left\{\mathfrak{X}_{a l t} \kappa_{1}, \kappa_{2}\right\}$ where $\square \mathfrak{X}_{a l t_{1}^{\kappa_{1}, \kappa_{2}}}$ is the set of $\square$ subformulae of $\varphi$. If $\varphi$ is unsatisfiable in Kalt $_{1}^{\kappa_{1}, \kappa_{2}}$ then $\Pi_{\mathfrak{X}}^{\text {alt }}{\text { ( } 1, \kappa_{2}}^{(\varphi)}$ is unsatisfiable in first-order logic.

Proof. Let $M=\operatorname{Cls}\left(\operatorname{Corr}\left(\left\{a l t_{1}^{\kappa_{1}, \kappa_{2}}\right\}\right) \wedge \Pi(\varphi)\right)$ and $N=\operatorname{Cls}\left(\Pi_{\mathfrak{X}}^{a l t_{1}^{\kappa_{1}, \kappa_{2}}}(\varphi)\right) . \quad M$ and $N$ are identical, except that $M$ contains the clause (20), and $N$ contains instances of the clausal schema

$$
\neg R^{\kappa_{1}}(x, y) \vee \neg Q_{\neg \square \psi}(y) \vee \neg R^{\kappa_{2}}(x, z) \vee Q_{\square \neg \psi}(z),
$$

for each $\square \psi \in \operatorname{Sf}(\varphi)$, the positive shortcut clauses $Q_{\sim \psi}(x) \vee Q_{\psi}(x)$, and the definitional clauses for $\square \neg \psi$ formulae appearing in the clausal schema instances.

The idea of the proof is the same as before. For simplicity we assume a particular strategy is used in the derivations starting with $M$. Let $\mathrm{H}_{a l t}{ }_{1}^{\kappa_{1}, \kappa_{2}}$ be a procedure based on $\mathrm{H}$ in which the order of the application is the following. Inferences with hyperresolution, factoring and splitting have highest priority, then inferences with the theory clause (20) are performed, followed by inferences with the paramodulation rule on state (unary) unit clauses. Inferences with the paramodulation rule on non-unary unit clauses are not essential for deriving the empty clause. The rule of reflexivity resolution is not applicable, since $M$ does not include negative equality literals and no derived clause contains a negative

ACM Transactions on Computational Logic, Vol. X, No. Y, August 2005. 
occurrence of equality. The strategy of $\mathrm{H}_{a l t_{1}^{\kappa_{1}, \kappa_{2}}}$ is fair. Therefore, $\mathrm{H}_{a l t} \kappa_{1}^{\kappa_{1}, \kappa_{2}}$ is complete by Theorem B.1.

Our goal is to show the following.

$\left(P a l t_{1}^{\kappa_{1}, \kappa_{2}}\right)$ If $M_{0}, M_{1}, \ldots, M_{n}$ is a branch in a $\mathrm{H}_{a l t_{1}, \kappa_{2}}$ derivation from $M_{0}=M$, then it is possible to construct a branch $N_{0}, N_{1}, \ldots, N_{k}$ in an $\mathrm{H}$-derivation starting from $N_{0}=N$, for some $k$, and the following are true.

(1) Every positive ground state clause in $M_{n}$ occurs also in $N_{k}$.

(2) For every clause $t \approx u$ in $M_{n}$, there is a set of clauses

$$
\left\{R^{\kappa_{1}}\left(s, v_{1}\right), R\left(v_{1}, t\right), R^{\kappa_{2}}\left(s, v_{2}\right), R\left(v_{2}, u\right)\right\}
$$

in $M_{n} \cap N_{k}$, with $s, v_{1}, v_{2}$ ground terms such that $Q_{\neg \square \psi}\left(v_{1}\right) \in M_{n} \cap N_{k}$ and $t=f_{\neg \square \psi}\left(v_{1}\right)$ for some $\psi$.

The base case and Case 2 are straightforward.

Case 1 and 4: $C$ is a definitional clause. In all cases the positive premises are unary units which belong to both $M_{n}$ and $N_{k}$ by the induction hypothesis. Thus, we let $k^{\prime}=k+1$ and $N_{k^{\prime}}=N_{k} \cup\{D\}$, which is obtained by the same inference as $M_{n+1}$ from $M_{n}$. Clearly, this preserves the property $\left(P_{a l t}^{\kappa_{1}, \kappa_{2}}\right)$.

Case 3.1: $C$ is the clause (20) and the conclusion $D$ is derived with the positive premises $\left\{R^{\kappa_{1}}\left(s, v_{1}\right), R\left(v_{1}, t\right), R^{\kappa_{2}+1}(s, u)\right\} \subseteq M_{n}$. Thus, $D=(t \approx u)$, with $t$ and $u$ ground terms. Noting that all transition clauses have the form $R(v, f(v))$ for some Skolem function $f$ associated with a $\neg \square \psi$ formula, it is not difficult to verify that there must be a subformula $\psi$ such that $Q_{\neg \square \psi}\left(v_{1}\right) \in M_{n}$ and $t=f_{\neg \square \psi}\left(v_{1}\right)$. By the induction hypothesis: $\left\{R^{\kappa_{1}}\left(s, v_{1}\right), R\left(v_{1}, t\right), R^{\kappa_{2}+1}(s, u), Q_{\neg \square \psi}\left(v_{1}\right)\right\} \subseteq N_{k}$. Thus we let $k^{\prime}=k$ and $N_{k^{\prime}}=N_{k}$. Clearly, property $\left(P a l t_{1}^{\kappa_{1}, \kappa_{2}}\right)$ is preserved.

Since $t \approx u$ cannot be derived from $N_{k}$ it is important to ensure the existence of inferences from $N_{k}$ that produce all (unit) state clauses derivable with equality clauses from $M_{n}$. This is done in the next case.

Case 3.2: $C$ is a (unit) equality $t \approx u$. Then $t$ and $u$ are ground terms. Suppose the other premise has the form $Q_{\phi}(t)$ and the conclusion is $D=Q_{\phi}(u)$. We now describe how $Q_{\phi}(u)$ can be derived from $N_{k}$. By the induction hypothesis there is a ground term $s$ which satisfies the property $\left(P a l t_{1}^{\kappa_{1}, \kappa_{2}} .2\right)$. I.e. there is a subset

$$
M^{\prime}=\left\{R^{\kappa_{1}}\left(s, v_{1}\right), R\left(v_{1}, t\right), R^{\kappa_{2}}\left(s, v_{2}\right), R\left(v_{2}, u\right), Q_{\neg \square \psi}\left(v_{1}\right)\right\}
$$

of $M_{n}$ and $t=f_{\neg \square \psi}\left(v_{1}\right)$, for some $\psi$. By the induction hypothesis, $M^{\prime} \subseteq N_{k}$. Hence, the following clauses can be derived from $N_{k}$ :

$$
\begin{array}{ll}
Q_{\neg \psi}(t) & \text { using the appropriate definitional clause for } Q_{\neg \square \psi} \\
Q_{\square \neg \psi}\left(v_{2}\right) & \text { using the clausal schema }(21) \\
Q_{\neg \psi}(u) & \text { using the definitional clause for } Q_{\square \neg \psi}, \text { and } R\left(v_{2}, u\right)
\end{array}
$$

The link between $D=Q_{\phi}(u)$ and $Q_{\neg \psi}(u)$ is: Either (i) $\phi$ is a subformula of $\neg \psi$ which does not occur below a modal operator in $\neg \psi$, or (ii) there is a clause $Q_{\square \vartheta}\left(v_{2}\right)$ in $M_{n}$ and $\phi$ is a subformula of $\vartheta$ which does not occur below a modal operator in $\vartheta$. In the case (i), $Q_{\phi}(u)$ can be derived from $N_{k}$ using $Q_{\neg \psi}(u)$ and Boolean definitional clauses. In the case (ii), $Q_{\vartheta}(u)$ can be derived using $Q_{\square \vartheta}\left(v_{2}\right)$ and $R\left(v_{2}, u\right)$. Then $Q_{\phi}(u)$ can be derived from $N_{k}$ using $Q_{\vartheta}(u)$ and Boolean definitional clauses.

The lengths of the subderivations described above are in all cases bounded.

Inspection of the proof reveals that the positive shortcut clauses are superfluous for completeness proof and may be omitted from the definition of the translation for $\mathrm{Kalt}_{1}^{\kappa_{1}, \kappa_{2}}$. The next section considers logics where shortcut clauses appear to be essential. 


\section{E.3 Completeness for $K \oplus \diamond^{+} \square p \rightarrow \square p$}

Let $5^{\kappa}=\diamond^{\kappa} \square p \rightarrow \square p$, or equivalently, $\neg \square^{\kappa} \neg \square p \rightarrow \square p$, where $\kappa \geq 1$. The clausal schema for $5^{\kappa}$ used in the axiomatic encoding is

$$
\neg R^{\kappa}(x, y) \vee \neg Q_{\square \psi}(y) \vee Q_{\square \psi}(x) .
$$

Theorem E.6 $K 5^{\kappa}$ is complete with respect to the class of frames satisfying (the universal closure of)

$$
\neg R^{\kappa}(x, y) \vee \neg R(x, z) \vee R(y, z) .
$$

Proof. By Sahlqvist's [1975] Theorem.

Lemma E.7 Let $N$ be a set of clauses including

$$
\begin{array}{ll}
\text { 1. } \neg Q_{\square \neg \psi}(x) \vee \neg R(x, y) \vee Q_{\neg \psi}(y) & \text { definitional clause for } Q_{\square \neg \psi} \\
\text { 2. } Q_{\square \neg \psi}(x) \vee Q_{\neg \square \neg \psi}(x) & \text { shortcut between } \square \neg \psi \text { and } \neg \square \neg \psi \\
\text { 3. } \neg Q_{\psi}(x) \vee \neg Q_{\neg \psi}(x) & \text { shortcut between } \psi \text { and } \neg \psi
\end{array}
$$

If $\left\{R(s, t), Q_{\psi}(t)\right\} \subseteq N$ then $Q_{\neg \square \neg \psi}(s)$ can be derived in two steps.

Proof. Using 1,2 , and $R(s, t)$ we can derive $Q_{\neg \square \neg \psi}(s) \vee Q_{\neg \psi}(t) . Q_{\neg \square \neg \psi}(s)$ is then a hyperresolvent with 3 and $Q_{\psi}(t)$.

Lemma E.8 Let $N$ be a set of clauses including

$$
\begin{array}{ll}
\text { 1. } \neg Q_{\psi}(x) \vee \neg Q_{\neg \psi}(x) & \text { shortcut between } \psi \text { and } \neg \psi \\
\text { 2. } \neg Q_{\square \psi}(x) \vee \neg R(x, y) \vee Q_{\psi}(y) & \text { definitional clause for } Q_{\square \psi} \\
\text { 3. } \quad Q_{\square \psi}(x) \vee Q_{\neg \square \psi}(x) & \text { shortcut between } \square \psi \text { and } \neg \square \psi
\end{array}
$$

If $\left\{R(s, t), Q_{\neg \psi}(t)\right\} \subseteq N$ then $Q_{\neg \square \psi}(s)$ can be derived in two inference steps.

Proof. First, use 2, 3, and $R(s, t)$ to derive $Q_{\neg \square \psi}(s) \vee Q_{\psi}(t)$. Resolving this clause and $Q_{\neg \psi}(t)$ with 1 produces $Q_{\neg \square \psi}(s)$.

Lemma E.9 $\mathfrak{X}_{\varphi}^{5^{\kappa}}=\mathfrak{X}_{\varphi}^{\epsilon} \cup \bigcup_{i=0}^{\kappa-1} \square^{i} \neg \square \mathfrak{X}_{\varphi}^{\epsilon}$.

Proof. Not difficult, if we note $\square \mathfrak{X}_{\varphi}^{5^{\kappa}}=\square \mathfrak{X}_{\varphi}^{\epsilon} \cup \bigcup_{i=1}^{\kappa} \square^{i} \neg \square \mathfrak{X}_{\varphi}^{\epsilon}$.

Lemma E.10 Let $\varphi$ be any modal formula and let $N=\operatorname{Cls}\left(\Pi_{\mathfrak{X}}^{5^{\kappa}}(\varphi)\right)$ be the clausal form of the axiomatic encoding with respect to $\mathfrak{X}=\mathfrak{X}_{\varphi}^{5^{\kappa}}$. Let $N_{k}$ be an endpoint of a derivation from $N$ by $\mathrm{H}$.

(1) For any $1 \leq i \leq \kappa$, suppose $\left\{R^{i}(s, t), Q_{\square \psi}(t)\right\} \subseteq N_{k}$. Then (i) $Q_{\neg \square^{i} \neg \square \psi}(s)$ can be derived in $2 i$ inference steps. (ii) If $i=\kappa$ then $Q_{\square \psi}(s)$ can be derived in one inference step.

(2) If $\left\{R^{\kappa}(s, t), Q_{\neg \square \psi}(s)\right\} \subseteq N_{k}$ then $Q_{\neg \square \psi}(t)$ can be derived in two inference steps.

(3) If $\left\{Q_{\neg \square^{\kappa} \neg \square \psi}(s)\right\} \subseteq N_{k}$ then $Q_{\square \psi}(s)$ can be derived in $2 \kappa+1$ inference steps.

(4) Suppose $\left\{R^{i}(s, u), R(u, t), Q_{\neg \square^{i} \neg \square \psi}(s)\right\} \subseteq N_{k}$ where $1 \leq i<\kappa$, and $s$ and $u$ occur on a path of length $\kappa$. Then (i) $Q_{\neg \square^{\kappa} \neg \square \psi}(u)$ can be derived in $O(\kappa)$ inference steps, and (ii) $Q_{\square \psi}(u)$ can be derived in $O(\kappa)$ further inference steps.

ACM Transactions on Computational Logic, Vol. X, No. Y, August 2005. 
Proof. (1.i) The derivation of $Q_{\neg \square^{i} \neg \square \psi}(s)$ with $R^{i}(s, t)$ and $Q_{\square \psi}(t)$ uses the derivations in Lemmas E.7 and E.8.

(1.ii) To derive $Q_{\square \psi}(s)$ with $R^{\kappa}(s, t)$ and $Q_{\square \psi}(t)$, use the clausal schema instance for $5^{\kappa}$.

For (2), use the clauses

$$
\begin{aligned}
& \text { 1. } \neg Q_{\square \psi}(y) \vee \neg R^{\kappa}(x, y) \vee Q_{\square \psi}(x) \quad \text { clausal schema instance } \\
& \text { 2. } Q_{\square \psi}(x) \vee Q_{\neg \square \psi}(x) \quad \text { shortcut between } \square \psi \text { and } \neg \square \psi \\
& \text { 3. } \neg Q_{\square \psi}(x) \vee \neg Q_{\neg \square \psi}(x) \quad \text { shortcut between } \square \psi \text { and } \neg \square \psi
\end{aligned}
$$

to derive
4. $Q_{\neg \square \psi}(t) \vee Q_{\square \psi}(s)$
5. $Q_{\neg \square \psi}(t)$
using $1,2, R(s, t)$
using $3,4, Q_{\neg \square \psi}(s)$.

For (3), use the definitional clauses for $Q_{\neg \square^{\kappa} \neg \square \psi}, Q_{\neg \square^{\kappa-1} \neg \square \psi}, \ldots, Q_{\neg \square \neg \square \psi}$, which have the form $(1 \leq i \leq \kappa)$

$$
\begin{aligned}
& \neg Q_{\neg \square^{i} \neg \square \psi}(x) \vee R\left(x, f_{i}(x)\right) \\
& \neg Q_{\neg \square^{i} \neg \square \psi}(x) \vee Q_{\square^{i} \neg \square \psi}\left(f_{i}(x)\right)
\end{aligned}
$$

(where $f_{i}$ is abbreviating notation for $f_{\neg \square^{i} \neg \square \psi}$ ) together with the clauses of the form

$$
Q_{\square^{i} \neg \square \psi}(x) \vee Q_{\neg \square^{i} \neg \square \psi}(x) \quad \text { shortcut between } \square^{i} \neg \square \psi \text { and } \neg \square^{i} \neg \square \psi
$$

to derive the following clauses in sequence

$$
\begin{aligned}
& R\left(s, f_{\kappa}(s)\right), \quad Q_{\square^{\kappa} \neg \square \psi}\left(f_{\kappa}(s)\right) \\
& R\left(f_{\kappa}(s), f_{\kappa-1}\left(f_{\kappa}(s)\right)\right), \quad Q_{\square_{\kappa-1} \neg \square \psi}\left(f_{\kappa-1}\left(f_{\kappa}(s)\right)\right), \\
& \quad \vdots \\
& R\left(f_{2}\left(\cdots f_{\kappa}(s) \cdots\right), f_{1}\left(\cdots f_{\kappa}(s) \cdots\right)\right), \quad Q_{\square \neg \square \psi}\left(f_{1}\left(\cdots f_{\kappa}(s) \cdots\right)\right) .
\end{aligned}
$$

Now derive $Q_{\square \psi}(s)$ as in (1) with the transition clauses.

For (4.i), first assume the beginning of the path through $s$ and $u$ is $s$. Thus, assume the path is represented by $R^{\kappa}(s, w)$. Then use (2) to get $Q_{\neg \square^{i} \neg \square \psi}(w)$. Now propagate $Q_{\neg \square^{\kappa} \neg \square \psi}$ to $u$ by repeatedly using Lemma E.8. Second, assume the beginning of the path through $s$ and $u$ is a predecessor $v$ of $s$. Then propagate $Q_{\neg \square_{\neg \square \psi}}$ to $v$ using Lemma E.7 where $i<j<\kappa$. If the path is represented by $R^{\kappa}(v, w)$ then, again use (2) to get $Q_{\neg \square^{j} \neg \square \psi}(w)$. Thus we can get $Q_{\neg \square^{\kappa} \neg \square \psi}(u)$ by repeatedly using Lemma E.8.

(4.ii) is a consequence of (i) and (3).

Inspection reveals that the clauses used in all of the above derivations are present in $N$.

Fork relatives. Let $N$ be a clause set. We say $s$ is a $(n, m)$-fork relative of $t$ in $N$ $(n, m \geq 0)$ iff $N$ includes a subset

$$
F=\left\{R\left(u_{0}, u_{1}\right), \ldots, R\left(u_{n-1}, u_{n}\right)\right\} \cup\left\{R\left(v_{0}, v_{1}\right), \ldots, R\left(v_{m-1}, v_{m}\right)\right\}
$$

of transition clauses such that $u_{0}=v_{0}, u_{n}=s$ and $v_{m}=t$. The set $F$ is called an $(n, m)$-fork relating $s$ and $t$. The root of the fork is the state $u_{0}$. The path from $u_{0}$ to $s$ is called the $s$-branch and the path from $u_{0}$ to $t$ is called the $t$-branch. The difference value $d$ of $F$ is defined to be the difference between the length of the $t$-branch and the $s$-branch in $F$, modulo $\kappa$, in particular, $m \bmod \kappa=(n+d) \bmod \kappa$. 
$(3,1)$

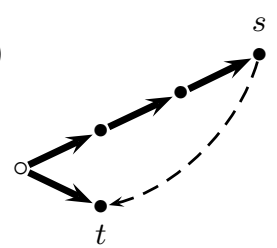

$(1,2)$
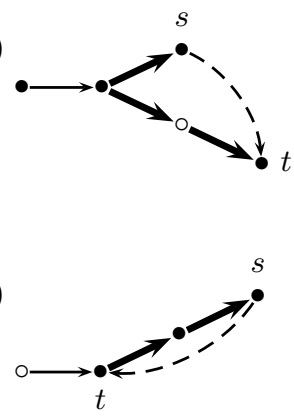
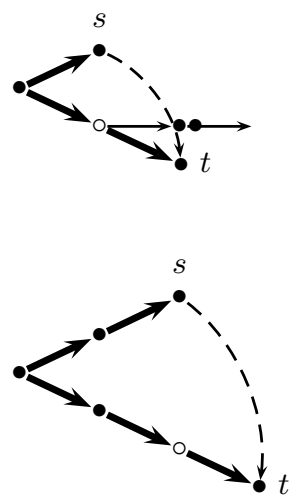

$(2,3)$

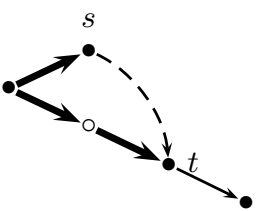

Fig. 10. Admissible $(n, m)$-forks relating $s$ and $t$ for $5^{3}$

For example, if $\left\{R(s, s), R\left(t, t^{\prime}\right), R(u, v), R(v, s), R(v, t)\right\} \in N$ then $s$ is a $(0,0)$-fork relative of itself, $t$ is a $(0,1)$-fork relative of $t^{\prime}, s$ is a $(1,1)$-fork relative of $t$ (via the root $v$ ), but also a $(2,2)$-fork relative (via the root $u$ ), and $s$ is both a $(1,2)$-fork relative and a $(2,3)$-fork relative of $t^{\prime}$.

Admissible fork for $5^{\kappa}$. If $F \subseteq N$ is an $(n, m)$-fork relating $s$ and $t$ and the following is satisfied then we say $F$ is a fork admissible for $5^{\kappa}$.

(1) $m \bmod \kappa=(n+1) \bmod \kappa$, i.e. the difference value of $F$ is one.

(2) $s$ is not a predecessor of $t$.

(3) $t$ has an immediate predecessor $u$.

(4) There is a path through $u$ and the root of $F$ whose length is divisible by $\kappa$.

Suppose $\kappa=3$. Figure 10 gives examples of admissible forks of two elements $s$ and $t$ for $5^{3}$. The immediate predecessor world $u$ of $t$ is indicated by $\circ$. The fat arrows represent the fork, while the other arrows represent additional links which form part of the path through the root of the fork and the immediate predecessor of $t$, as required by condition (4). No proper subgraph of one of the depicted graphs can be an admissible $5^{3}$ forks relating $s$ and $t$.

Lemma E.11 Let $\varphi$ be any modal formula and let $N=\operatorname{Cls}\left(\Pi_{\mathfrak{X}}^{5^{\kappa}}(\varphi)\right)$ be the clausal form of the axiomatic encoding with respect to $\mathfrak{X}=\mathfrak{X}_{\varphi}^{5^{\kappa}}$. Let $N_{k}$ be the endpoint of a partial derivation from $N$ by $\mathrm{H}$. Let $s$ be a $(n, m)$-fork relative of $t$ in $N_{k}$ and suppose $Q_{\square \psi}(s) \in N_{k}$. Then $Q_{\square \psi}(u)$ can be derived from $N_{k}$ if the fork is admissible and $u$ is the immediate predecessor of $t$.

Proof. Using Lemma E.10.(1) it is not difficult to see that $Q_{\neg \square^{i} \neg \square \psi}(v)$ can be derived for some $i, 1 \leq i \leq \kappa$, where $v$ denotes the root of the fork. Using Lemmas E.10.(2) 
and E.10.(4.ii) we can now show that $Q_{\square \psi}(u)$ can be derived. Clearly the total number of inference steps involved is always finitely bounded.

Gluing together forks. For the purposes of the completeness proof we need to glue together admissible forks to build particular subframes of models of $N$. First, we establish that all models of $N$ are tree models.

Lemma E.12 Let $\varphi$ be any modal formula and let $N=\operatorname{Cls}\left(\Pi_{\mathfrak{X}}(\varphi)\right)$ be the clausal form of the axiomatic encoding with respect to some $\mathfrak{X}$. All Herbrand models of $N$ generated by $\mathrm{H}$ are tree models.

Proof. Each transition clause in any $\mathrm{H}$-derivation has the form $R(s, f(s))$. So, $f(s)$ can be interpreted as an $R$-successor of $s$. Furthermore, $f(s)$ is not an $R$-successor of any $t \neq s$ and for each term $f(s)$ there is a literal $R(s, f(s))$ that can be derived.

Lemma E.13 Let $U$ be a set of transition clauses determining a tree structure. Suppose $F \subset U$ is an $(n, m)$-fork relating $s$ and $t$ and $v$ is the root of $F$. Let $d$ be the difference value of $F$.

(1) Then the extension $F^{\prime}=\left\{R^{i}(u, v)\right\} \cup F$, for any $i \geq 1$, is an $(n+i, m+i)$-fork of $s$ and $t$ and the difference value of $F^{\prime}$ is $d$. Further, if $F$ is admissible then $F^{\prime}$ is admissible.

(2) If $R^{i}(t, w) \in U$ then $s$ is an $(n, m+i)$-fork relative of $w$ and the difference value of the fork is $(d+i) \bmod \kappa$.

(3) If $R^{i}(u, s) \in U$ then $u$ is a fork relative of $t$ and the difference value of the fork is $(d+i) \bmod \kappa$.

(4) If $F^{\prime} \subseteq U$ is an $\left(n^{\prime}, m^{\prime}\right)$-fork relating $u$ and $s$ with difference value $d^{\prime}$ then $F \cup F^{\prime}$ is a fork relating $u$ and $t$ with difference value $\left(d+d^{\prime}\right) \bmod \kappa$.

(5) $F$ is an $(m, n)$-fork relating $t$ and $s$, and the difference value is $(-d) \bmod \kappa$.

(6) If $R^{i}(s, u) \in U$ then $t$ is a fork relative of $u$ and the difference value of the fork is $(-d+i) \bmod \kappa$.

(7) If $F^{\prime} \subseteq U$ is an $\left(n^{\prime}, m^{\prime}\right)$-fork relating $s$ and $u$ with difference value $d^{\prime}$ then $F \cup F^{\prime}$ is a fork relating $t$ and $u$ with difference value $\left(-d+d^{\prime}\right) \bmod \kappa$.

Proof. (1) Not difficult.

(2) $w$ is a successor of $t$, hence the result follows easily.

(3) If $s$ has no predecessor in $F$ then the result is clear. Suppose $s$ has a predecessor $s^{\prime}$ such that $R^{i}\left(s^{\prime}, s\right)$. Then it follows that $u$ and $s^{\prime}$ coincide, for otherwise $U$ does not determine a tree structure. Then $u$ is an $(n-i, m)$-fork relative of $t$ and hence the difference value of the fork is $d+i$. Finally suppose the root $v$ of $F$ is such that $R^{i-n}(v, s)(i>n)$. Then $u$ is a predecessor of $v$ such that $R^{i-n}(u, v)$. Hence $u$ is a $(0, m+(i-n))$-fork relative of $t$. The difference value is $(d+i) \bmod \kappa$, which is equal to $m+(i-n) \bmod \kappa$.

(4) If $m^{\prime}=n$ then, since $U$ is a tree structure, the $s$-branch of $F$ and the $s$-branch of $F^{\prime}$ coincide. Thus the union $F^{\prime \prime}$ of the $u$-branch of $F$ and the $t$-branch of $F^{\prime}$ is a fork relating $u$ and $t$. Then $F^{\prime \prime}$ is an $\left(n^{\prime}, m\right)$-fork relating $u$ and $t$ with $F^{\prime \prime} \subseteq F \cup F^{\prime}$. Hence $F \cup F^{\prime}$ is also an $\left(n^{\prime}, m\right)$-fork relating $u$ and $t$. It is not difficult to see that the difference value of $F^{\prime \prime}$ and $F \cup F^{\prime}$ is $\left(d+d^{\prime}\right) \bmod \kappa$.

If $m^{\prime}>n$ then the $s$-branch of $F$ is a suffix of the $s$-branch of $F^{\prime}$. By (1), the fork relating $s$ and $t$ via the root of $F^{\prime}$ is a $\left(n+\left(m^{\prime}-n\right), m+\left(m^{\prime}-n\right)\right)$-fork with difference value $d$. Now argue as for the previous case.

The case that $n>m^{\prime}$ is analogous.

(5) Not difficult. 
(6) Use (5) and (2).

(7) The argument is similar as for (4), except that the orientation of $F^{\prime}$ is different.

Theorem E.14 (Completeness of $\Pi_{\mathfrak{X}}^{5^{\kappa}}$ ) Let $\varphi$ be any modal formula and assume $\mathfrak{X}=$ $\left\{\mathfrak{X}_{5^{\kappa}}\right\}$ where $\mathfrak{X}_{5^{\kappa}}=\mathfrak{X}_{\varphi}^{5^{\kappa}}$. If $\varphi$ is unsatisfiable in $K 5^{\kappa}$ then $\Pi_{\mathfrak{X}}^{5^{\kappa}}(\varphi)$ is unsatisfiable in first-order logic.

Proof. Suppose $M=\operatorname{Cls}\left(\operatorname{Corr}\left(\left\{5^{\kappa}\right\}\right) \wedge \Pi(\varphi)\right)$ and $N=\operatorname{Cls}\left(\Pi_{\mathfrak{X}}^{5^{\kappa}}(\varphi)\right)$. Our goal is to show the following.

$\left(P 5^{\kappa}\right)$ If $M_{0}, M_{1}, \ldots, M_{n}$ is a branch in an $\mathrm{H}$-derivation from $M_{0}=M$, then it is possible to construct a branch $N_{0}, N_{1}, \ldots, N_{k}$ in an $\mathrm{H}$-derivation starting from $N_{0}=N$, for some $k$, and the following are true.

(1) Every state clause in $M_{n}$ occurs also in $N_{k}$.

(2) For every clause $R(s, t)$ in $M_{n}$ but not in $N_{k}$, there is an admissible fork $F$ relating $s$ and $t$, and if $Q_{\square \psi}(s) \in N_{k}$, then $Q_{\square \psi}(u) \in N_{k}$ where $u$ is the immediate predecessor of $t$ in $F$.

The base case, Case 1 and Case 3 are the same as in the proof of Theorem D.1.

Case 3: $C$ is the clause (23) and $D$ is derived with positive premises in $M_{n}$. Suppose the positive premises are

$$
V=\left\{R^{\kappa}(v, s), R(v, t)\right\}
$$

Then $D=R(s, t)$. Observe $R(s, t)$ cannot be derived from $N_{k}$. We show the existence of inferences that ensure that the properties of $\left(P 5^{\kappa}\right)$ are true. Property $\left(P 5^{\kappa} .1\right)$ holds trivially by the induction hypothesis. For property $\left(P 5^{\kappa} .2\right)$ the derivation from $N_{k}$ needs to be continued in such a way that, if $Q_{\square \psi}(s)$ is an arbitrary unit in $N_{k}$ which is associated with a $\square$ formula, then there is a clause $Q_{\square \psi}\left(v^{\prime}\right)$ in $N_{k}$ such that $v^{\prime}$ is a predecessor of $t$ in $N_{k}$.

Not all of the clauses in $V$ need to be present in $N_{k}$. Suppose $U$ is any subset of $V$, $U \subseteq N_{k}$ but $(V-U) \cap N_{k}=\emptyset$. We need to show that $t$ has an immediate predecessor $v^{\prime}$ such that $Q_{\square \psi}\left(v^{\prime}\right) \in N_{k}$.

The case that $U=V$ is easiest. I.e. suppose $\left\{Q_{\square \psi}(s), R^{\kappa}(v, s), R(v, t)\right\} \subseteq N_{k}$. Then $s$ is a $(\kappa, 1)$-fork relative of $t$. It follows from Lemma E.11 that it is possible to derive $Q_{\square \psi}(v)$. Therefore let $N_{k^{\prime}}$ be the result of this derivation from $N_{k}$ with $k^{\prime} \geq k$. Evidently, property $\left(P 5^{\kappa} .2\right)$ is true for $N_{k^{\prime}}$.

Suppose $U$ is a proper subset of $V$. For each $R(u, w)$ in $V$ but absent from $U$, by the induction hypothesis there is an admissible fork relating $u$ and $w$. In particular, the difference value is one. Making use of Lemmas E.13.(2), E.13.(3) and E.13.(4) we can show that there is a fork $F^{\prime}$ relating $v$ (the root) and $s$ in $N_{k}$. The difference value of $F^{\prime}$ is $(\kappa \cdot 1) \bmod \kappa=0$. Now, suppose $R(v, t) \in N_{k}$ then by Lemma E.13.(6) there is a fork $F$ relating $s$ and $t$ with difference value one. If on the other hand $R(v, t) \notin N_{k}$, then there is a fork $F^{\prime \prime} \subseteq N_{k}$ relating $v$ and $t$ with difference value one. Now use Lemma E.13.(7) to conclude there is a fork $F$ relating $s$ and $t$ with difference value one. Ignoring the property (4) of the definition of admissibility, in both cases $F$ is admissible.

Clearly, if $F$ is admissible and if $v^{\prime}$ is the immediate predecessor of $t$ in $F$ then by Lemma E.11 it is possible to derive $Q_{\square \psi}\left(v^{\prime}\right)$. Then we can let $N_{k^{\prime}}$ be the endpoint of the derivation of $Q_{\square \psi}\left(v^{\prime}\right)$ from $N_{k}$.

Therefore, it remains to prove for $F$ that there is a path through $v^{\prime}$ and the root of $F$ whose length is divisible by $\kappa$. This can be done by inspection.

Case 4: $C$ is a definitional clause associated with a $\square$ subformula, i.e. $C=\neg Q_{\square \psi}(x) \vee$ $\neg R(x, y) \vee Q_{\psi}(y)$. Assume $D$ is derived with $C$ and two premises $Q_{\square \psi}(s)$ and $R(s, t)$ 
in $M_{n}$. By the induction hypothesis, $Q_{\square \psi}(s)$ is also in $N_{k}$. If $R(s, t) \in N_{k}$ then we let $k^{\prime}=k+1$ and $N_{k^{\prime}}=N_{k} \cup\{D\}$, which is obtained by the same inference as $M_{n+1}$ from $M_{n}$.

If $R(s, t) \notin N_{k}$ then, by the induction hypothesis, $N_{k}$ contains a fork $F$ relating $s$ and $t$ such that $t$ has an immediate predecessor $u$ and $Q_{\square \psi}(u) \in N_{k}$. Since $R(u, t) \in N_{k}, D$ can be derived from $N_{k}$ using $C$. Therefore, we can let $N_{k^{\prime}}=N_{k} \cup\{D\}$ and $k^{\prime}=k+1$.

Notice that shortcut clauses were used in Lemmas E.7, E.8 and E.10.

\section{F. PROOFS OF COMPLETENESS FOR COMBINATIONS OF AXIOMS}

\section{F.1 Combinations of $T, B$ and 4}

Theorem F.1 Let $\varphi$ be any modal formula and assume $\mathfrak{X}=\left\{\mathfrak{X}_{T}, \mathfrak{X}_{4}\right\}$, where $\mathfrak{X}_{T}=\mathfrak{X}_{4}=$ $\mathfrak{X}_{\varphi}^{\epsilon}$. Then, $\Pi_{\mathfrak{X}}^{\mathcal{A}}(\varphi)$ is unsatisfiable in first-order logic, whenever $\varphi$ is unsatisfiable in $S 4$.

Proof. The proof is a simple combination of the proofs for $T$ and 4 in Theorem D.2. The property to be proved is the same as $\left(P_{4}\right)$. The positive shortcut clauses are not essential.

Theorem F.2 Let $\varphi$ be any modal formula and assume $\mathfrak{X}=\left\{\mathfrak{X}_{T}, \mathfrak{X}_{B}\right\}$, where $\mathfrak{X}_{T}=$ $\mathfrak{X}_{B}=\mathfrak{X}_{\varphi}^{\epsilon}$. Then, $\Pi_{\mathfrak{X}}^{\mathcal{A}}(\varphi)$ is unsatisfiable in first-order logic, whenever $\varphi$ is unsatisfiable in $K T B$.

Proof. The proof is a simple combination of the proofs for $T$ and $B$ (Theorems D.2 and D.1). The property to be proved is the same as $(P B)$. The positive shortcut clauses are not essential.

Theorem F.3 Let $\varphi$ be any modal formula and assume $\mathfrak{X}=\left\{\mathfrak{X}_{4}, \mathfrak{X}_{B}\right\}$, where $\mathfrak{X}_{4}=$ $\mathfrak{X}_{\varphi}^{\epsilon}$ and $\mathfrak{X}_{B}=\mathfrak{X}_{\varphi}^{4}$. Then, $\Pi_{\mathfrak{X}}^{4, B}(\varphi)$ is unsatisfiable in first-order logic, whenever $\varphi$ is unsatisfiable in $K_{4} B$.

Proof. By simulation of the corresponding modal reduction of Kracht [2001] on the first-order level.

Theorem F.4 Let $\varphi$ be any modal formula and assume $\mathfrak{X}=\left\{\mathfrak{X}_{T}, \mathfrak{X}_{B}, \mathfrak{X}_{4}\right\}$, where $\mathfrak{X}_{T}=$ $\mathfrak{X}_{4}=\mathfrak{X}_{\varphi}^{\epsilon}$ and $\mathfrak{X}_{B}=\mathfrak{X}_{\varphi}^{4}$. Then, $\Pi_{\mathfrak{X}}^{T, 4, B}(\varphi)$ is unsatisfiable in first-order logic, whenever $\varphi$ is unsatisfiable in $S 5$.

ProOF. By simulation of the modal reduction of [Kracht 2001].

In the argument of the last two proofs the positive shortcut clauses are needed for simulating applications of the modus ponens rule. This means a separate argument is required to show cut elimination. However, the example $K 4 B \vdash 5$ can be used to show that positive shortcut clauses are in fact essential for the completeness of both $\Pi_{\mathfrak{X}}^{4, B}$ and $\Pi_{\mathfrak{X}}^{T, 4, B}$.

\section{F.2 Combinations involving $D$}

Theorem F.5 Let $\varphi$ be any modal formula and assume $\mathfrak{X}=\left\{\mathfrak{X}_{D}, \mathfrak{X}_{B}\right\}$, where $\mathfrak{X}_{D}=$ $\mathfrak{X}_{B}=\mathfrak{X}_{\varphi}^{\epsilon}$. Then, $\Pi_{\mathfrak{X}}^{\mathcal{A}}(\varphi)$ is unsatisfiable in first-order logic, whenever $\varphi$ is unsatisfiable in $K D B$.

Proof. The proof can be seen to a combination of the proofs of Theorems D.1 and D.3. As in the proof of Theorem D.3 the proof is by an induction over derivations from the set $M=\operatorname{Cls}(\operatorname{Corr}(\{D, B\}) \wedge \Pi(\varphi))$ exhibiting that it is possible to construct a simulating 
derivation from $N=\operatorname{Cls}\left(\Pi_{\mathfrak{X}}^{D, B}(\varphi)\right)$ and a function $h$ from state clauses in the first derivation to state clauses in the second derivation. Suppose $f$ is the Skolem function of the seriality clause. The property to be proved is:

$(P D B)$ If $M_{0}, M_{1}, \ldots, M_{n}$ is a branch in the $\mathrm{H}$-derivation from $M_{0}=M$, then it is possible to construct a branch $N_{0}, N_{1}, \ldots, N_{k}$ in an H-derivation starting from $N_{0}=N$, for some $k$ with $2 n \geq k \geq n$, and the following are true.

(1) For each state clause $C$ in $M_{n}, N_{k}$ contains the state clause $h(C)$.

(2) For each transition clause of the form $R(s, g(s))$ in $M_{n}$ (where $g$ denotes $f$ or another Skolem function), $R(h(s), h(g(s)))$ is in $N_{k}$.

(3) For every transition clause $R(s, t)$ in $M_{n}$, if $R(h(s), h(t))$ is not in $N_{k}$, then $N_{k}$ contains the transition clause $R(h(t), h(s))$.

(4) For every ground term $f(s)$ in $M_{n}, h(f(s))=g(h(s))$ for some Skolem function $g$ in $N$.

The theory clauses of $M$ are the seriality clause and the symmetry clause. Their $\mathrm{H}$-closure will contain in addition the clause (we call this clause the converse seriality clause):

$$
R(f(x), x) \text {. }
$$

The base case and Cases 1 and 2 are the same as in Theorem D.3. Cases 3 and 4 are modifications of the relevant cases in Theorems D.1 and D.3.

Case 3: $C$ is the symmetry clause. There are three possibilities. (a) $D$ is derived with the seriality clause, i.e. $D$ is the converse seriality clause (24). (b) $D$ is derived with the converse seriality clause, i.e. $D$ is the seriality clause. (c) $D$ is derived with a transition clause $R(s, t)$, i.e. $D=R(t, s)$. In the first two cases property $(P D B)$ is trivially true since neither the seriality clause nor its converse can be derived from $N$ and neither are transition clauses. In the third case, we proceed similar as in the proof of Theorem D.1. (c.i) Suppose $\{R(h(s), h(t)), h(D)\} \subseteq N_{k}$. Then there is nothing to prove. (c.ii) Suppose $R(h(s), h(t)) \in N_{k}$ but $h(D) \notin N_{k}$. Since $h(D)$ is the converse of a clause in $N_{k}$ the property $(P D B)$ holds. (c.iii) Suppose $R(h(s), h(t)) \notin N_{k}$. Then by the induction hypothesis $h(D)=R(h(t), h(s)) \in N_{k}$ and the property $(P D B)$ holds. In all cases let $k^{\prime}=k$ and $N_{k^{\prime}}=N_{k}$.

Case 4: $C$ is a $\square$ definitional clause $\neg Q_{\square \psi}(x) \vee \neg R(x, y) \vee Q_{\psi}(y)$. Assume $D$ is derived with $C$, a state clause $Q_{\square \psi}(s)$ and a clause $R(s, t)$ in $M_{n}$. By the induction hypothesis, $Q_{\square \psi}(h(s)) \in N_{k}$. (a) If $R(s, t)$ is a transition clause and it has the form $R(s, g(s))$, then $D=Q_{\psi}(g(s))$ and by the induction hypothesis $R(h(s), h(g(s))) \in N_{k}$. Hence let $k^{\prime}=k+1$ and $N_{k^{\prime}}=N_{k} \cup\left\{Q_{\psi}(h(g(s)))\right\}$, which is obtained by the same inference as $M_{n+1}$ from $M_{n}$.

(b) If $R(s, t)$ is a transition clause but does not have the form $R(s, g(s))$. Then, $R(s, t)$ has the form $R(g(s), s)$ and $R(h(g(s)), h(s)) \notin N_{k}$. D is the clause $Q_{\psi}(s)$. By the induction hypothesis $R(h(s), h(g(s))) \in N_{k}$. Hence, $Q_{\psi}(h(s))$ can be derived from $N_{k}$ using $Q_{\square \psi}(h(g(s))), R(h(s), h(g(s)))$ and the $B$ schema clause instance $\neg R(x, y) \vee \neg Q_{\square \psi}(y) \vee$ $Q_{\psi}(x)$. Therefore, let $N_{k^{\prime}}=N_{k} \cup\left\{Q_{\psi}(h(s))\right\}$ and $k^{\prime}=k+1$.

(c) Suppose $R(s, t)$ is the seriality clause and $D=Q_{\psi}(f(s))$. Use $Q_{\square \psi}(h(s))$ and the $D$ schema clause instance $\neg Q_{\square \psi}(x) \vee Q_{\neg \square \neg \psi}(x)$ to derive $Q_{\neg \square \neg \psi}(h(s))$. Then derive $Q_{\psi}(g(h(s)))$ in one hyperresolution inference step with the first of the definitional clauses associated with $\neg \square \neg \psi$. Hence let $k^{\prime}=k+2$ and $N_{k^{\prime}}=N_{k} \cup\left\{Q_{\neg \square \neg \psi}(h(s)), Q_{\psi}(g(h(s)))\right\}$. Now let $h(f(s))=g(h(s))$.

(d) Suppose $R(s, t)$ is the converse seriality clause. For an inference to be possible it must be that $s=f(t)$ and $D=Q_{\psi}(t) . Q_{\square \psi}(h(f(t))) \in N_{k}$ and by the induction hypothesis $h(f(t))=g(h(t))$ for some Skolem function $g$ in $N$. I.e. $Q_{\square \psi}(g(h(t))) \in N_{k}$. It follows that there is an $Q_{\neg \square \neg \phi}(h(t))$ in $N_{k}$ leading directly or indirectly to the derivation of the

ACM Transactions on Computational Logic, Vol. X, No. Y, August 2005. 
clause $Q_{\square \psi}(g(h(t))) \in N_{k}$ via an inference between $Q_{\neg \square \neg \phi}(h(t))$ and the first existential definitional clause. With the second existential definitional clause the transition clause $R(h(t), g(h(t)))$ can be derived if it is not already in $N_{k}$. Now use the B schema clause instance to derive $Q_{\psi}(h(t))$. Hence let $k^{\prime}=k+1$ or $k^{\prime}=k+2$ as appropriate and $N_{k^{\prime}}=N_{k} \cup\left\{R(h(t), g(h(t))), Q_{\psi}(h(t))\right\}$.

In each case the property $(P D B)$ is satisfied.

Theorem F.6 Let $\varphi$ be any modal formula and assume $\mathfrak{X}=\left\{\mathfrak{X}_{D}, \mathfrak{X}_{4}\right\}$, where $\mathfrak{X}_{D}=$ $\mathfrak{X}_{4}=\mathfrak{X}_{\varphi}^{\epsilon}$. Then, $\Pi_{\mathfrak{X}}^{\mathcal{A}}(\varphi)$ is unsatisfiable in first-order logic, whenever $\varphi$ is unsatisfiable in KD4.

ProOF. The idea of the proof is as for Theorems D.3 and F.5 incorporating ingredients of the proof of Theorem D.2. Using a function $h$ between state clauses of the derivations, the proof shows that the existence of a refutation preserving mapping of derivations from the set $M=\operatorname{Cls}(\operatorname{Corr}(\{D, 4\}) \wedge \Pi(\varphi))$ to derivations from the set $N=\operatorname{Cls}\left(\Pi_{\mathfrak{X}}^{D, 4}(\varphi)\right)$. The property to be proved is the following.

(PD4) If $M_{0}, M_{1}, \ldots, M_{n}$ is a branch in the $\mathrm{H}$-derivation from $M_{0}=M$, then it is possible to construct a branch $N_{0}, N_{1}, \ldots, N_{k}$ in an H-derivation starting from $N_{0}=N$, for some $k$, and the following are true.

(1) For each state clause $C$ in $M_{n}, N_{k}$ contains the state clause $h(C)$.

(2) For every transition clause $R(s, t)$ in $M_{n}$ with $s \neq t$, if $R(h(s), h(t))$ is not in $N_{k}$, then

(a) $N_{k}$ contains a chain of transition clauses

$$
R\left(u_{1}, u_{2}\right), R\left(u_{2}, u_{3}\right), \ldots, R\left(u_{m-1}, u_{m}\right), R\left(u_{m}, u_{m+1}\right)
$$

such that $u_{1}=h(s)$ and $u_{m+1}=h(t)$, and

(b) if $Q_{\square \psi}(h(s)) \in N_{k}$, then $Q_{\square \psi}\left(u_{i}\right) \in N_{k}$ for every $i, 1 \leq i \leq m$.

(c) If $t=f^{l}(v)$ where $f$ is the Skolem function of the seriality clause then $h(t)=g\left(u_{m}\right)$ for some Skolem function $g$ in $N$.

Observe that the transition clauses derivable from $N$ have the form $R(s, g(s))$. The closure of the theory clauses of $M$, namely the seriality clause and the transitivity clause, are clauses of the form $R\left(x, f^{l}(x)\right)$. The transition clauses derivable from $M$ but not from $N$ include clauses of the form $R\left(s, f^{l}(t)\right)$ as well as $R(s, g(t))$ where $s$ and $t$ do not need to be identical.

The base case and Cases 1 and 2 of the inductive step are the same as in Theorem D.3. We therefore consider only the remaining cases without giving every detail.

Case 3: $C$ is the transitivity clause. (a) Suppose the positive premises are transition clauses of the form $R(s, v), R(v, t)$. Then $D=R(s, t)$. (a.i) Suppose $Q_{\square \psi}(h(s)) \in N_{k}$ and $\{R(h(s), h(v)), R(h(v), h(t))\} \subseteq N_{k}$. Then, unless $Q_{\square \psi}(h(v))$ does not already belong to $N_{k}$, it can be derived by one hyperresolution inference step using $Q_{\square \psi}(h(s))$, the clause $R(h(s), h(v))$, and the appropriate schema 4 clause instance.

(a.ii) Suppose one of $R(h(s), h(v))$ or $R(h(v), h(t))$, or both, are not in $N_{k}$. It is not difficult to see that using the induction hypothesis there must be a chain

$$
R\left(u_{1}, u_{2}\right), R\left(u_{2}, u_{3}\right), \ldots, R\left(u_{m-1}, u_{m}\right), R\left(u_{m}, u_{m+1}\right)
$$

of transition clauses in $N_{k}$ with $m \geq 2$, such that $u_{1}=h(s)$ and $u_{m+1}=h(t)$. Now, if $Q_{\square \psi}(h(s)) \in N_{k}$ we can iteratively derive $Q_{\square \psi}\left(u_{l}\right)$, for each $l$ such that $1 \leq l \leq m$ using the appropriate schema 4 clause instance. This shows that it possible to generate a set $N_{k^{\prime}}$ in boundedly many steps and satisfies the desired properties.

(b) Suppose the positive premises of an inference step with the transitivity clause are a transition clause $R(s, v)$ and a clause $R\left(x, f^{l}(x)\right)$. In addition suppose that $D=$ 
$R\left(v, f^{l}(v)\right)$. Then either (b.i) $R(h(s), h(v))$ belongs to $N_{k}$, or (b.ii) there is a chain (25) of transition clauses in $N_{k}$ with $m \geq 2$, such that $u_{1}=h(s)$ and $u_{m+1}=h(v)$. Since (b.i) can be regarded as a subcase of the case (b.ii) we consider (b.ii) only. If $Q_{\square \psi}(h(s)) \in N_{k}$ then by induction $Q_{\square \psi}\left(h\left(u_{m}\right)\right) \in N_{k}$ which can be propagated with the schema 4 clause so that we get $Q_{\square \psi}(h(v))$. Consecutively apply the schema D clause and the two existential definitional clause for $\neg \square \neg \psi$ to obtain $R\left(u_{m}, g\left(u_{m}\right)\right.$ and $Q_{\psi}\left(g\left(u_{m}\right)\right)$. Therefore, let $h\left(f^{l}(v)\right)=g\left(u_{m}\right)$ and verify that $(P D 4)$ holds for the appropriate $N_{k^{\prime}}$.

(c) Suppose the positive premises of an inference step with a clause $R\left(x, f^{l}(x)\right)$ and a transition clause $R(s, v)$ such that $s$ is unifiable with $f^{l}(x)$. Then proceed in a similar vain as in (b).

(d) If the positive premises are both of the form $R\left(x, f^{l}(x)\right)$ then the property is $(P D 4)$ is vacuously true.

Case 4: $C$ is a $\square$ definitional clause $\neg Q_{\square \psi}(x) \vee \neg R(x, y) \vee Q_{\psi}(y)$. Assume $D$ is derived with $C$, a state clause $Q_{\square \psi}(s)$ and a clause $R(s, t)$ in $M_{n}$. By the induction hypothesis, $Q_{\square \psi}(h(s)) \in N_{k}$. (a) $R(s, t)$ is a transition clause and $R(h(s), h(t)) \in N_{k}$. Then $D=Q_{\psi}(t)$. Let $k^{\prime}=k+1$ and $N_{k^{\prime}}=N_{k} \cup\{h(D)\}$, which is obtained by the same inference as $M_{n+1}$ from $M_{n}$.

(b) $R(s, t)$ is a transition clause and $R(h(s), h(t)) \notin N_{k}$. Then $D=Q_{\psi}(t)$. By the induction hypothesis, $N_{k}$ contains a chain $R\left(u_{1}, u_{2}\right), \ldots, R\left(u_{m-1}, u_{m}\right), R\left(u_{m}, u_{m+1}\right)$, such that $u_{1}=h(s)$ and $u_{m+1}=h(t)$, and $Q_{\square \psi}\left(u_{i}\right) \in N_{k}$ for all $i, 1 \leq i \leq m$. In particular, $Q_{\square \psi}\left(u_{m}\right) \in N_{k}$ and $R\left(u_{m}, h(t)\right) \in N_{k}$. Hence, $h(D)$ can be derived from $N_{k}$ using $C$.

(c) $R(s, t)$ is not a transition clause. Then it must be a clause of the form $R\left(x, f^{l}(x)\right)$ and $D=Q_{\psi}\left(f^{l}(s)\right)$. Now use $Q_{\square \psi}(h(s))$ and the $D$ schema clause to derive first $Q_{\neg \square \neg \psi}(h(s))$, and then $R\left(h(s), g(h(s))\right.$ with the existential definitional clause, and finally $Q_{\psi}(g(h(s)))$ with $C$. Let $h\left(f^{l}(s)\right)=g(h(s))$ and $N_{k^{\prime}}$ is the extension of $N_{k}$ with the clauses just derived.

In each case the property $\left(P D_{4}\right)$ is satisfied.

Inspection reveals that the positive shortcut clauses are inessential for proving the completeness of the reductions.

\section{F.3 Mixed reductions}

Theorem F.7 Let $\varphi$ be any modal formula and assume $\mathfrak{X}=\left\{\mathfrak{X}_{4}\right\}$, where $\mathfrak{X}_{4}=\mathfrak{X}_{\varphi}^{\epsilon}$. Then, $\operatorname{Corr}(\{T, B\}) \wedge \Pi_{\mathfrak{X}}^{4}(\varphi)$ is unsatisfiable in first-order logic, whenever $\varphi$ is unsatisfiable in S5.

Proof. By an argument similar to that used in the proof of Theorem D.2.

Theorem F.8 Let $\varphi$ be any modal formula and assume $\mathfrak{X}=\left\{\mathfrak{X}_{\mathcal{A}}\right\}$, where $\mathfrak{X}_{\mathcal{A}}=\mathfrak{X}_{\varphi}^{\epsilon}$ and $\mathcal{A} \in\{B, 4\}$. Then, $\operatorname{Corr}(\{D\}) \wedge \Pi_{\mathfrak{X}}^{\mathcal{A}}(\varphi)$ is unsatisfiable in first-order logic, whenever $\varphi$ is unsatisfiable in $K D \mathcal{A}$.

Proof. The proofs are similar to (and easier than) those of Theorems F.5 and F.6.

\section{G. PROOF OF SMALL MODEL PROPERTY, MODEL GENERATION}

Theorem G.1 Let $L$ be a non-serial and non-functional modal logic considered in Section 5 or a fusion of these. If a modal formula $\varphi$ is $L$-satisfiable then (i) it is satisfiable in a model with at most $2^{\mathcal{O}(|\varphi|)}$ elements, i.e. $L$ has the small model property, and (ii) resolution can be used to construct an $L$-model for $\varphi$.

Proof. Let $I$ be the Herbrand model defined by the ground unit clauses on an open branch in a $\mathrm{H}$-derivation for the axiomatic translation of $\varphi$. This model is only a skeleton

ACM Transactions on Computational Logic, Vol. X, No. Y, August 2005. 
model and the idea of the proof is to show that it can be expanded into a model $I^{\prime}$ such that $I \subseteq I^{\prime}$ and $I^{\prime}$ is a model of the classical translation of $\varphi$. For the logics we consider $I^{\prime}$ can be constructed by completing $I$, considered as a set of positive, ground unit clauses, with respect to the frame correspondence properties of $L$. The completion can be done with $H$. Since the correspondence properties in $\operatorname{Corr}(\Delta)$ reduce to range-restricted, functionfree and equality-free clauses, the completion process only adds transition clauses which are ground and do not contain new Skolem terms. We need to prove that the modal model corresponding to $I^{\prime}$ is indeed a standard $L$-model for $\varphi$. The case analyses in these proofs is analogous to the case analyses in the proofs of the completeness of the axiomatic translation for $L$, even though the argument goes in the opposite direction.

Since the Skolem terms occurring in $I$ and $I^{\prime}$ are the same, the modal models corresponding to $I$ and $I^{\prime}$ have the same number of states. The number of Skolem terms in $I$ is bounded by $2^{\mathcal{O}(|\varphi|)}$. Finally prove that the small model property is preserved when fusing the logics, or use [Kracht and Wolter 1997]. It is also routine to prove that the generation of $I^{\prime}$ for fusions of modal logics can be done in the way described above.

\section{ACKNOWLEDGMENTS}

Work on the topic of this paper started in 1999 when we recognised that the translation mapping described in an unpublished manuscript of Hans de Nivelle (1999) can be situated in the framework of Ohlbach [1998]. We thank Hans de Nivelle, Dmitry Tishkovsky and the anonymous referees for valuable comments. A substantial part of this research was conducted during a six month visit by the first author to the Max-Planck-Institut für Informatik in Saarbrücken in 2002. Support by the MPI and the UK Engineering and Physical Sciences Research Council (research grants GR/M36700 and GR/M88761/01) is gratefully acknowledged.

\section{REFERENCES}

Bachmair, L. AND Ganzinger, H. 2001. Resolution theorem proving. In Handbook of Automated Reasoning, A. Robinson and A. Voronkov, Eds. Elsevier, 19-99.

Bachmair, L., Ganzinger, H., and Waldmann, U. 1993. Superposition with simplification as a decision procedure for the monadic class with equality. In Proc. KGC'93. LNCS, vol. 713. Springer, 83-96.

Balbiani, P. And Herzig, A. 1994. A translation from the modal logic of provability into K4. $J$. Appl. Non-Classical Logics 4, 1, 73-78.

Blackburn, P., De Rijke, M., and Venema, Y. 2001. Modal Logic. Cambridge Univ. Press.

BoY DE LA Tour, T. 1992. An optimality result for clause form translation. J. Symbolic Computat. 14, 283-301.

Bry, F. AND YAhyA, A. 2000. Positive unit hyperresolution tableaux for minimal model generation. J. Automated Reasoning 25, 1, 35-82.

Castilho, M. A., Fariñas del Cerro, L., Gasquet, O., and Herzig, A. 1997. Modal tableaux with propagation rules and structural rules. Fundamenta Informaticae 3-4, 32, 281-297.

Chellas, B. F. 1980. Modal Logic: An Introduction. Cambridge Univ. Press.

De Giacomo, G. 1996. Eliminating "converse" from converse PDL. J. Logic, Language and Inform. 5, 2, 193-208.

De Nivelle, H. And de RiJke, M. 1999. Deciding the guarded frgament by resolution. To appear in J. Symbolic Computat.

De Nivelle, H., Schmidt, R. A., And Hustadt, U. 2000. Resolution-based methods for modal logics. Logic J. IGPL 8, 3, 265-292.

Demri, S. 2001. The complexity of regularity in grammar logics and related modal logics. $J$. Logic Computat. 11, 6, 933-960. 
DemRI, S. AND DE Nivelle, H. 2003. Deciding regular grammar logics with converse through first-order logic. Research Report LSV-03-4, Spécification et Vérification, CNRS \& ENS de Cachan, France.

Demri, S. And Goré, R. 1999. Tractable transformations from modal provability logics into first-order logic. In Automated Deduction-CADE-16. LNAI, vol. 1632. Springer, 16-30.

Demri, S. AND OrlowsKa, E. 1999. Every finitely reducible logic has the finite model property with respect to the class of $\diamond$-formulae. Studia Logica 62, 177-200.

Gabbay, D. M. 1975. Decidability results in non-classical logics. Ann. Math. Logic 8, 237-295.

Ganzinger, H. And De Nivelle, H. 1999. A superposition decision procedure for the guarded fragment with equality. In Proc. LICS'99. IEEE Computer Society, 295-303.

Ganzinger, H., Meyer, C., And De Nivelle, H. 1999. The two-variable guarded fragment with transitive relations. In Proc. LICS'99. IEEE Computer Society, 24-34.

Georgieva, L., Hustadt, U., And Schmidt, R. A. 2001. Computational space efficiency and minimal model generation for guarded formulae. In Proc. LPAR 2001. LNAI, vol. 2250. Springer, $85-99$.

Georgieva, L., Hustadt, U., and Schmidt, R. A. 2002. A new clausal class decidable by hyperresolution. In Automated Deduction-CADE-18. LNAI, vol. 2392. Springer, 260-274.

Goldblatt, R. 1987. Logics of Time and Computation. CSLI Lecture Notes, vol. 7. Chicago Univ. Press.

Goranko, V. And Passy, S. 1992. Using the universal modality: Gains and questions. Journal of Logic and Computation 2, 1, 5-30.

Goré, R. 1999. Tableau methods for modal and temporal logics. In Handbook of Tableau Methods, M. D’Agostino, D. Gabbay, R. Hähnle, and J. Posegga, Eds. Kluwer, 297-396.

GrÄDEL, E. 1999. On the restraining power of guards. Journal of Symbolic Logic 64, 1719-1742.

Hughes, G. E. and Cresswell, M. J. 1996. A New Introduction to Modal Logic. Routledge, London.

Hustadt, U., Dixon, C., Schmidt, R. A., And Fisher, M. 2000. Normal forms and proofs in combined modal and temporal logics. In Proc. FroCoS 2000. LNAI, vol. 1794. Springer, 73-87.

Hustadt, U. And Schmidt, R. A. 1999a. An empirical analysis of modal theorem provers. J. Appl. Non-Classical Logics 9, 4, 479-522.

Hustadt, U. And Schmidt, R. A. 1999b. Maslov's class K revisited. In Automated Deduction$C A D E$-16. LNAI, vol. 1632. Springer, 172-186.

Hustadt, U. And SCHMidt, R. A. 1999c. On the relation of resolution and tableaux proof systems for description logics. In Proc. IJCAI'99. Morgan Kaufmann, 110-115.

Hustadt, U. and Schmidt, R. A. 2000a. Issues of decidability for description logics in the framework of resolution. In Automated Deduction in Classical and Non-Classical Logics. LNAI, vol. 1761. Springer, 191-205.

Hustadt, U. And Schmidt, R. A. 2000b. MSPASS: Modal reasoning by translation and first-order resolution. In Proc. TABLEAUX 2000. LNAI, vol. 1847. Springer, 67-71.

Hustadt, U. And Schmidt, R. A. 2002. Using resolution for testing modal satisfiability and building models. J. Automated Reasoning 28, 2, 205-232.

Kracht, M. 1995. Highway to the danger zone. J. Logic Computat. 5, 1, 93-109.

Kracht, M. 1999. Tools and Techniques in Modal Logic. Studies in Logic, vol. 142. Elsevier.

Kracht, M. 2001. Reducing modal consequence relations. J. Logic Computat. 11, 6, 879-907.

Kracht, M. 2002. Notes on the space requirements for checking satisfiability in modal logics. In Advances in Modal Logic, Vol. 4, P. Balbiani, N.-Y. Suzuki, and F. Wolter, Eds. King's College Publications, London, 243-264.

Kracht, M. and Wolter, F. 1997. Simulation and transfer results in modal logic-a survey. Studia Logica 59, 2, 149-177.

Leitsch, A. 1997. The Resolution Calculus. EATCS Texts in Theoretical Computer Science. Springer.

Letz, R. And Stenz, G. 2001. DCTP: A disconnection calculus theorem prover. In Automated Reasoning - IJCAR 2001. LNAI, vol. 2083. Springer, 381-385.

ACM Transactions on Computational Logic, Vol. X, No. Y, August 2005. 
Lewis, H. R. 1980. Complexity results for classes of quantificational formulas. J. Computer and System Sci. 21, 317-353.

Lutz, C. 1999. Complexity of terminological reasoning revisited. In Proc. LPAR'99. LNAI, vol. 1705. Springer, 181-200.

Lutz, C. And Sattler, U. 2002. The complexity of reasoning with boolean modal logics. In $A d-$ vances in Modal Logics Volume 3, F. Wolter, H. Wansing, M. de Rijke, and M. Zakharyaschev, Eds. CSLI Publications, Stanford.

MassaCCI, F. 2000. Single step tableaux for modal logics: Computational properties, complexity and methodology. J. Automated Reasoning 24, 3, 319-364.

Nonnengart, A., Ohlbach, H. J., And Szalas, A. 1997. Quantifier elimination for second-order predicate logic. To appear in Logic, Language and Reasoning: Essays in honour of Dov Gabbay, Kluwer.

OhlBACh, H. J. 1998. Combining Hilbert style and semantic reasoning in a resolution framework. In Automated Deduction-CADE-15. LNAI, vol. 1421. Springer, 205-219.

Ohlbach, H. J., Nonnengart, A., De Rijke, M., and Gabbay, D. 2001. Encoding two-valued nonclassical logics in classical logic. In Handbook of Automated Reasoning, A. Robinson and A. Voronkov, Eds. Elsevier, 1403-1486.

Plaisted, D. A. and Greenbaum, S. 1986. A structure-preserving clause form translation. J. Symbolic Computat. 2, 293-304.

Riazanov, A. And Voronkov, A. 1999. Vampire. In Automated Deduction-CADE-16. LNAI, vol. 1632. Springer, 292-296.

Robinson, A. And Voronkov, A., Eds. 2001. Handbook of Automated Reasoning. Elsevier.

SAHLQVist, H. 1975. Completeness and correspondence in the first and second order semantics for modal logics. In Proc. Scandinavian Logic Symposium, 1973, S. Kanger, Ed. North-Holland, $110-143$.

Schmidt, R. A. And Hustadt, U. 2000. A resolution decision procedure for fluted logic. In Automated Deduction-CADE-17. LNAI, vol. 1831. Springer, 433-448.

Schmidt, R. A. And Hustadt, U. 2003. Mechanised reasoning and model generation for extended modal logics. In Theory and Applications of Relational Structures as Knowledge Instruments. LNCS, vol. 2929. Springer, 38-67.

Schulz, S. 2002. E: A Brainiac theorem prover. J. AI Comm. 15, 2-3, 111-126.

Socher-Ambrosius, R. And Johann, P. 1997. Deduction Systems. Graduate Texts in Computer Science. Springer, New York.

Stenz, G. 2002. DCTP 1.2: System abstract. In Proc. TABLEAUX 2002. LNAI, vol. 2381. Springer, 335-340.

Weidenbach, C., Brahm, U., Hillenbrand, T., Keen, E., Theobald, C., and Topic, D. 2002. SPASS version 2.0. In Automated Deduction-CADE-18. LNAI, vol. 2392. Springer, 275-279.

Received October 2004; accepted August 2005 